NBER WORKING PAPER SERIES THE WELFARE EFFECTS OF VERTICAL INTEGRATION IN MULTICHANNEL
TELEVISION MARKETS

\author{
Gregory S. Crawford \\ Robin S. Lee \\ Michael D. Whinston \\ Ali Yurukoglu \\ Working Paper 21832 \\ http://www.nber.org/papers/w21832 \\ NATIONAL BUREAU OF ECONOMIC RESEARCH \\ 1050 Massachusetts Avenue \\ Cambridge, MA 02138 \\ December 2015, Revised April 2017
}

We would like to thank the editor, five anonymous referees, and numerous individuals and seminar participants for helpful comments; and ESRC Grant RES-062-23-2586 (Crawford), the NYU Stern Center for Global Economy and Business (Lee), and the Toulouse Network for Information Technology and the NSF (Whinston) for support. All errors are our own. The views expressed herein are those of the authors and do not necessarily reflect the views of the National Bureau of Economic Research.

At least one co-author has disclosed a financial relationship of potential relevance for this research. Further information is available online at http://www.nber.org/papers/w21832.ack

NBER working papers are circulated for discussion and comment purposes. They have not been peer-reviewed or been subject to the review by the NBER Board of Directors that accompanies official NBER publications.

(C) 2015 by Gregory S. Crawford, Robin S. Lee, Michael D. Whinston, and Ali Yurukoglu. All rights reserved. Short sections of text, not to exceed two paragraphs, may be quoted without explicit permission provided that full credit, including $\odot$ notice, is given to the source. 
The Welfare Effects of Vertical Integration in Multichannel Television Markets

Gregory S. Crawford, Robin S. Lee, Michael D. Whinston, and Ali Yurukoglu

NBER Working Paper No. 21832

December 2015, Revised April 2017

JEL No. L13,L42,L51,L82

\section{ABSTRACT}

We investigate the welfare effects of vertical integration of regional sports networks (RSNs) with programming distributors in U.S. multichannel television markets. Vertical integration can enhance efficiency by reducing double marginalization and increasing carriage of channels, but can also harm welfare due to foreclosure and incentives to raise rivals' costs. We estimate a structural model of viewership, subscription, distributor pricing, and affiliate fee bargaining using a rich dataset on the U.S. cable and satellite television industry (2000-2010). We use these estimates to analyze the impact of simulated vertical mergers and divestitures of RSNs on competition and welfare, and examine the efficacy of regulatory policies introduced by the U.S. Federal Communications Commission to address competition concerns in this industry.

Gregory S. Crawford

University of Zurich

gregory.crawford@econ.uzh.ch

Robin S. Lee

Department of Economics

Harvard University

Littauer Center 120

Cambridge, MA 02138

and NBER

robinlee@fas.harvard.edu
Michael D. Whinston

Sloan School of Management

and Department of Economics

Massachusetts Institute of Technology

100 Main St

Cambridge, MA 02142

and NBER

whinston@mit.edu

Ali Yurukoglu

Graduate School of Business

Stanford University

Stanford, CA 94305

and NBER

ayurukog@stanford.edu 


\title{
The Welfare Effects of Vertical Integration in Multichannel Television Markets*
}

\author{
Gregory S. Crawford ${ }^{\dagger} \quad$ Robin S. Lee ${ }^{\ddagger} \quad$ Michael D. Whinston ${ }^{\S} \quad$ Ali Yurukoglu
}

April 3, 2017

\begin{abstract}
We investigate the welfare effects of vertical integration of regional sports networks (RSNs) with programming distributors in U.S. multichannel television markets. Vertical integration can enhance efficiency by reducing double marginalization and increasing carriage of channels, but can also harm welfare due to foreclosure and incentives to raise rivals' costs. We estimate a structural model of viewership, subscription, distributor pricing, and affiliate fee bargaining using a rich dataset on the U.S. cable and satellite television industry (2000-2010). We use these estimates to analyze the impact of simulated vertical mergers and divestitures of RSNs on competition and welfare, and examine the efficacy of regulatory policies introduced by the U.S. Federal Communications Commission to address competition concerns in this industry.
\end{abstract}

Keywords: vertical integration, foreclosure, double marginalization, raising rivals' costs, cable television

JEL: L13, L42, L51, L82

\section{Introduction}

The welfare effects of vertical integration is an important but controversial issue. The theoretical literature on the pro- and anti-competitive impacts of vertical integration is vast (cf. Perry, 1990; Rey and Tirole, 2007; Riordan, 2008; Bresnahan and Levin, 2013), and typically contrasts potential efficiencies related to the elimination of double marginalization (Spengler, 1950) and the alignment of investment incentives (Willamson, 1985; Grossman and Hart, 1986) with the potential for losses arising from incentives to foreclose rivals and raise their costs (Salop and Scheffman, 1983; Krattenmaker and Salop, 1986; Hart and Tirole, 1990; Ordover et al., 1990). Despite a growing literature, empirical evidence on the quantitative magnitudes of these potential effects, and the overall net welfare impact, is still limited.

\footnotetext{
${ }^{*}$ We would like to thank the editor, five anonymous referees, and numerous individuals and seminar participants for helpful comments; and ESRC Grant RES-062-23-2586 (Crawford), the NYU Stern Center for Global Economy and Business (Lee), and the Toulouse Network for Information Technology and the NSF (Whinston) for support. All errors are our own.

${ }^{\dagger}$ Department of Economics, University of Zurich; gregory.crawford@econ.uzh.ch

${ }^{\ddagger}$ Department of Economics, Harvard University; robinlee@fas.harvard.edu

${ }^{\S}$ Department of Economics and Sloan School of Management, MIT; whinston@mit.edu

${ }^{\top}$ Graduate School of Business, Stanford University; ayurukog@stanford.edu
} 
This paper quantifies the welfare effects of vertical integration in cable and satellite television in the context of high-value regional sports programming in the U.S. Whether the ownership of content by distributors harms welfare has been at the heart of the debate over recently approved (e.g., Comcast and NBC in 2011), abandoned (e.g., Comcast and Time Warner in 2015), and proposed (e.g., AT\&T and Time Warner in 2016) mergers in the television industry. The attention that these mergers have attracted is partly due to the industry's overwhelming reach and size: over $80 \%$ of the approximately 120 million television households in the U.S. subscribe to multichannel television, and the mean individual consumes about four hours of television per day. ${ }^{1}$ Regional sports programming is a large part of this industry, receiving $\$ 4.1$ billion out of over $\$ 30$ billion per year in negotiated affiliate fees paid by distributors to all content providers, and an additional $\$ 700$ million per year in advertising dollars. ${ }^{2}$

Our focus on the multichannel television industry, and in particular regional sports programming, is driven by several factors that create empirical leverage to address this question. First, there is significant variation across the industry in terms of ownership of regional sports content by cable and satellite distributors, also referred to as multichannel video programming distributors (MVPDs). Although this variation is primarily at the national-level for most channels, regional sports networks (RSNs) are present in smaller geographic areas, and thus there is useful variation in ownership patterns both across regions and over time. Additionally, the industry is the subject of significant regulatory and antitrust attention in addition to merger review, including the application of "program access rules" and exceptions to this rule, such as the "terrestrial loophole" which exempted certain distributors from supplying integrated content to rivals.

There are two key components of our analysis. The first is the construction of a comprehensive dataset on the U.S. multichannel television industry, collected and synthesized from numerous sources. The dataset comprises aggregate and individual-level consumer viewership and subscription patterns, channel ownership and integration status, and prices, quantities, and channel carriage "lineups" for cable and satellite bundles at the local market level for the years 2000 to 2010.

The second component is the specification and estimation of a structural model of the multichannel television industry that captures consumer viewership and subscription decisions, MVPD pricing and carriage decisions, and bargaining between MVPDs and content providers. We significantly extend the model of Crawford and Yurukoglu (2012) to construct an empirical framework suitable for the analysis of vertical integration and mergers. Our model incorporates integrated firms' incentives to foreclose rivals' access to inputs, the potential for double marginalization, and the possibility of imperfect coordination and internalization within an integrated firm. This last feature is one of the novel aspects of our approach, as we estimate, rather than impose, the degree to which firms internalize the profits of integrated units when distributors make pricing and channel carriage decisions, and channels decide to supply or foreclose rival distributors. Given our

\footnotetext{
${ }^{1}$ http://www.nielsen.com/us/en/insights/news/2016/nielsen-estimates-118-4-million-tv-homes-inthe-us--for-the-2016-17-season.html, http://www.nielsen.com/content/dam/corporate/us/en/reportsdownloads/2016-reports/q3-2016-total-audience-report.pdf, accessed on March 13, 2016.

${ }^{2}$ SNL Kagan.
} 
goal to evaluate whether vertical integration improves or worsens welfare due to improvements in internal efficiency or increases in foreclosure of rivals, taking this approach avoids building into our model the assumption that these two effects actually happen to the extent predicted by theory. For example, only very simple views of the firm imply that integrated firms behave as if they are under unitary control, and managers of integrated firms may well either not consider or over-react to the gains that can be reaped from foreclosure.

An important input into identifying these effects is our estimates of the change in distributor profits from the addition or removal of an RSN from any of its programming bundles. We use the relationship between distributors' market shares and channel carriage, as well as observed viewership patterns and negotiated affiliate fees, to infer the relative values consumers place on different channels. With the estimated profit effects in hand, the pro-competitive effects of vertical integration are largely identified from the degree to which RSN carriage is higher for integrated distributors conditional on the RSN's profitability to the distributor; the anti-competitive foreclosure effects are identified by lower RSN supply to downstream rivals of integrated RSNs.

We find that integrated distributors substantially but incompletely internalize the effects of their pricing and carriage decisions on their upstream channels' profits: we estimate that only $\$ 0.79$ of each dollar of profit realized by its integrated partner is internalized when an integrated MVPD makes pricing and carriage decisions, or when integrated MVPDs and RSNs bargain with each other. We also find that integrated RSNs fully (and perhaps more than fully) take into account the benefits their downstream divisions reap when a rival distributor is denied access to the RSN's programming.

After estimating our model, we leverage its structure to examine the mechanisms through which pro- and anti-competitive effects of vertical integration might occur. We do so by simulating vertical mergers and divestitures for 26 RSNs that were active in 2007, and examining their effects on equilibrium firm (carriage, pricing, affiliate fee bargaining and supply) and consumer (subscription, viewership) decisions. We consider integration scenarios when program access rules - which ensure that non-integrated rival distributors have access to integrated content - are effectively enforced, and when they are not. When program access rules are enforced, our counterfactual simulations capture the pro-competitive effects of integration from improved internalization of pricing and carriage decisions within the integrated firm. When program access rules are not enforced, our simulations allow as well for integrated (typically cable) distributors to engage in foreclosure, denying access to or charging higher prices for their owned RSN to non-integrated rival (typically satellite) distributors.

Our results highlight the importance of program access rules in determining the effects of vertical integration. In counterfactual simulations that enforce program access rules, we find that vertical integration leads to significant gains in both consumer and aggregate welfare. These benefits arise due to both lower cable prices (through the reduction of double marginalization) and greater carriage of the RSN. Averaging results across channels, we find that integration of a single RSN with effective program access rules in place would reduce average cable prices by $1.2 \%(\$ 0.67)$ per 
subscriber per month in markets served by the RSN, and increase overall carriage of the RSN by $9.4 \%$. Combined, these effects would yield, on average, a $\$ 0.43$ increase in total welfare from all television services, representing approximately $17 \%$ of the average consumer willingness to pay for a single RSN. We also predict that consumer welfare would increase.

When program access rules are instead not enforced, we find that - at the estimated lower bound for our "rival foreclosure" parameter - rival distributors would be denied access to an integrated RSN in 4 of out of 26 cases; for the other 22 cases, the rival distributors continue to have access but pay on average $18 \%$ higher affiliate fees than if program access rules were effectively enforced. Together, failure to enforce program access rules leads to a reduction in both consumer and total welfare of $1-2 \%$ of the average consumer willingness to pay for a single RSN. We find that the loss is significantly larger in cases in which the rival distributors are denied access. The foreclosure of satellite distributors tends to occur when the RSN is owned by a cable distributor whose market share is large in the geographic region served by the RSN. Our counterfactual results suggest that satellite distributors are excluded from carrying the RSN when the integrated cable distributor's share of households that it could serve exceeds approximately $85 \%$.

On net, we find that the overall effect of vertical integration in the absence of effective program access rules - allowing for both efficiency and foreclosure incentives - is to increase consumer and total welfare on average, resulting in (statistically significant) gains of approximately $\$ 0.38-0.39$ per household per month, representing $15-16 \%$ of the average consumer willingness to pay for an RSN. In the 4 markets in which rival distributors would be denied access, gains are quite small and cannot be distinguished from zero, while consumer and total welfare gains are positive and statistically significant in the 22 cases in which exclusion does not occur in response to vertical integration. Finally, stemming from the foreclosure and raising rivals' costs effects discussed above, rival distributors are predicted to be worse off; satellite surplus, in particular, falls $2.2 \%$ when vertical integration occurs with program access rules, and by $3.2 \%$ without these rules in place.

Despite the richness of our empirical model, the effects that we document are only partial. Most importantly, our model and analysis does not allow vertical integration to influence investments made by RSNs and MVPDs (both those that integrate and their rivals). ${ }^{3}$ As emphasized in the literature on investment effects of vertical integration (Bolton and Whinston, 1991; Hart, 1995), the direction of these effects on consumer and aggregate surplus are ambiguous a priori (and remain an important topic for future research).

Related Literature. Previous work studying the cable industry, including Waterman and Weiss (1996), Chipty (2001), and Chen and Waterman (2007), have primarily relied on reduced form cross-sectional analyses for a limited subset of channels and found that integrated cable systems are more likely to carry their own, as opposed to rival, content. ${ }^{4}$ An exception is Suzuki (2009)

\footnotetext{
${ }^{3}$ For example, we predict that cable-integration of an RSN always has a negative impact on satellite distributors; this raises the possibility that widespread integration by cable distributors of RSNs might impact satellite distributors' effectiveness as a competitor to cable to a greater extent than admitted in our analysis.

${ }^{4}$ Chipty (2001) also estimates a structural demand system to calculate integration effects on consumer welfare.
} 
who studies the 1996 merger between Time Warner and Turner broadcasting. His analysis uses time series variation in ownership, finding that vertically integrated channels were more likely to be carried post merger and rival non-integrated channels were less likely to be carried. ${ }^{5}$ These studies cannot, however, separate efficiency from foreclosure incentives, nor can they provide estimates of overall welfare effects. For example, reduced carriage of rival non-integrated channels could reflect either foreclosure effects or the impact of efficient increases in carriage of integrated channels when channels are substitutes. We complement this literature on vertical integration in the cable industry in two ways. First, building a structural model allows us to make welfare statements about the impact of vertical integration and identify the mechanisms through which the effects work. Second, we leverage a richer, panel dataset on consumer viewership and bundle subscription, and the pricing, carriage, and bargaining decisions of channels and distributors.

This paper also adds to the growing empirical literature on the effects of vertical integration and other vertical arrangements (e.g. Shepard, 1993; Hastings, 2004; Hastings and Gilbert, 2005; Hortacsu and Syverson, 2007; Villas-Boas, 2007; Mortimer, 2008; Houde, 2012; Lee, 2013; Conlon and Mortimer, 2015; Asker, 2016). We build on existing approaches by estimating a model that explicitly incorporates avenues for vertical integration to improve the efficiency of pricing and channel carriage decisions, and to generate foreclosure or raise costs of rival distributors; and by providing estimates of the degree to which integrated firms, in practice, act on each of these incentives. ${ }^{6}$ Using these estimates, we are then able to estimate the net welfare impact of vertical integration that trades off these pro- and anti-competitive effects. Finally, we develop methods for the estimation and simulation of counterfactual scenarios in vertical markets characterized by bilateral oligopoly and negotiated prices that can be applied in other related settings. ${ }^{7}$

Road Map. The rest of this paper is organized as follows. In the next section, we provide an overview of the U.S. cable and satellite industry and regional sports networks, and describe the data that we use in our analysis. We present our model of the industry in Section 3, and detail its estimation and our parameter estimates in Sections 4 and 5. We then assess the welfare effects of vertical integration by discussing the implementation of and findings from our counterfactual simulations in Section 6 and conclude in Section 7.

\section{Institutional Detail and Data}

Our study analyzes the U.S. cable and satellite industry for the years 2000 to 2010 and focuses on the ownership of "Regional Sports Networks" (RSNs) by cable and satellite distributors. In this section, we describe the industry's structure, RSNs, and regulatory policy during this period. We

\footnotetext{
${ }^{5}$ See also Caves et al. (2013) who provide evidence that RSN affiliate fees are correlated with downstream MVPD footprints.

${ }^{6}$ See also Michel (2013), who examines whether firms jointly maximize profits following a horizontal merger.

${ }^{7}$ E.g., Ho and Lee (2017) adapt techniques developed in this paper to examine hospital and insurance competition in health care markets.
} 
then discuss the data that we use to estimate the model. The tables referenced in this section are contained in Appendix A.

\subsection{Industry Structure}

In the time period that we study, the vast majority of households in the U.S. were able to subscribe to a multichannel television bundle from one of three downstream multichannel video programming distributors (MVPDs): a local cable company (e.g., Comcast, Time Warner Cable, or Cablevision) or one of two nationwide satellite companies (DirecTV or Dish Network). ${ }^{8}$ Cable companies transmit their video signals through a physical wire whereas satellite companies distribute video wirelessly through a south-facing satellite dish attached to a household's dwelling. The majority of distributors' revenue comes from selling subscriptions to three different bundles of programming: a limited basic bundle which retransmits over-the-air broadcast stations, an expanded basic bundle containing 40-60 of the most popular channels available on cable (e.g., AMC, CNN, Comedy Central, ESPN, MTV, etc.), and a digital bundle containing between 10 to 50 more, smaller, niche channels. ${ }^{9}$

Downstream distributors negotiate with content producers over the terms at which the distributors can offer the content producers' channels to consumers. These negotiations usually center on a monthly per subscriber "affiliate fee" that the downstream distributor pays the channel for every subscriber who has access to the channel, whether the subscriber watches it or not. ${ }^{10}$

\subsection{Regional Sports Networks}

RSNs carry professional and college sports programming in a particular geographic region. For example, the New England Sports Network (NESN) carries televised games of the Boston Red Sox and the Boston Bruins. Metropolitan areas can have multiple RSNs. For example, in the New York City metropolitan area, there are four different RSNs: Madison Square Garden (MSG), MSG Plus, SportsNet NY, and Yankees Entertainment and Sports (YES). Some RSNs also serve multiple metropolitan areas. For example, the Sun Sports network holds the rights to the Miami Heat and the Tampa Bay Rays, amongst others.

According to industry estimates, RSNs command the second-highest per subscriber affiliate

\footnotetext{
${ }^{8}$ Telephone MVPDs (primarily consisting of AT\&T and Verizon) did not enter a significant number of markets until 2007, at which point combined they had approximately 1.2 million subscribers according to financial filings; by the end of 2010, they had 6.9 million out of 100.8 million total MVPD subscribers (FCC, 2013).

${ }^{9}$ Our analysis focuses on the provision of multichannel programming, and does not explicitly consider the bundling or sale of Internet or phone services by cable or satellite distributors. In 2007, according to the U.S. Census Bureau Service Annual Survey, cable and other program distributors received $60 \%$ of their revenues from multichannel programming distribution services, $16 \%$ of revenues from Internet access services, and $6 \%$ from telephony services; other revenue sources included air time and installation and rental of equipment. During our sample period, Internet access (telephony) services grew from 2\% (1\%) of cable distributor revenues in 2000 to $17 \%$ (8\%) by 2010 while programming revenues fell from $77 \%$ to $57 \%$.

${ }^{10}$ As discussed in Crawford and Yurukoglu (2012), payments between distributors and content providers are primarily in the form of linear fees; fixed fee monetary transfers are rare, and if they exist, they are typically neglible relative to the total payment.
} 
fees after the national sports network ESPN. For example in 2010 Comcast SportsNet (CSN) Philadelphia had per subscriber monthly fees that averaged $\$ 2.85$ per month whereas highly-rated national channels such as Fox News, TNT, and USA were around $\$ 1$ per subscriber per month (and ESPN over $\$ 4$ per subscriber per month).

RSNs are sometimes owned by entities that also own downstream cable or satellite distributors. Figure 1 shows RSNs' ownership affiliations with downstream distributors over a 13-year period for the RSNs in our data that were active in 2007. Many RSNs are owned, to some degree, by a downstream distributor. For example, in 2007, downstream distributors had ownership interests in 16 of these RSNs. The cable MVPDs that owned RSNs are Comcast, Cablevision, Cox, and Time Warner. DirecTV, the largest satellite operator (and second-largest U.S. MVPD), indirectly had stakes in numerous RSNs through its partial owners News Corporation and Liberty Media Corporation. ${ }^{11}$ Ownership affiliations also vary over time, as RSNs may be (partially) acquired, divested, or sold to other distributors.

\subsection{Regulatory Policy}

There are several key features of the regulatory environment for RSNs, and vertically integrated content more generally, that are pertinent for our study. During our sample period, vertically integrated firms were subject to the "Program Access Rules" (PARs), which required that vertically integrated content be made available to rival distributors at non-discriminatory prices (subject to final-offer arbitration if necessary). The PARs only applied to content that was transmitted to MVPDs via satellite. This covered all national cable channels (which need satellite transmission to cost-effectively reach cable systems around the country) and most RSNs. However, a handful of RSNs transmitted their signal terrestrially (usually via microwave), thereby avoiding the jurisdiction of the PARs. This was called the "terrestrial loophole" in the Program Access regulation. In 2007, only two long-standing cable-integrated RSNs were able to leverage the terrestrial loophole: Comcast SportsNet in Philadelphia and SD4 in San Diego (owned by Cox Cable); in both cases, the channel was not provided to satellite distributors. ${ }^{12}$ As a result, Major League Baseball (MLB), National Basketball Association (NBA), and National Hockey League (NHL) games in Philadelphia were only available on cable and not on DirecTV or Dish Network. Similarly in San Diego, MLB games were available only through cable. This feature of regulatory history will be an important source of identifying variation in our econometric estimation.

The PARs were introduced in 1992 and required renewal by the FCC every five years. They were allowed to lapse in 2012 and replaced by rules giving the Commission the right to review any programming agreement for anti-competitive effects on a case-by-case basis under the "unfair acts"

\footnotetext{
${ }^{11}$ News Corporation and Liberty Media both had a partial ownership stake in DirecTV starting in 2003; at the beginning of 2008, News Corporation completed an asset swap with Liberty in which News traded its stake in DirectTV for Liberty's stake in News.

${ }^{12}$ Time Warner Cable also employed the terrestrial loophole from 2006 to 2008 for the (then relatively new) Charlotte Bobcats NBA franchise by placing some their games on News 14, a terrestrially delivered regional news channel.
} 


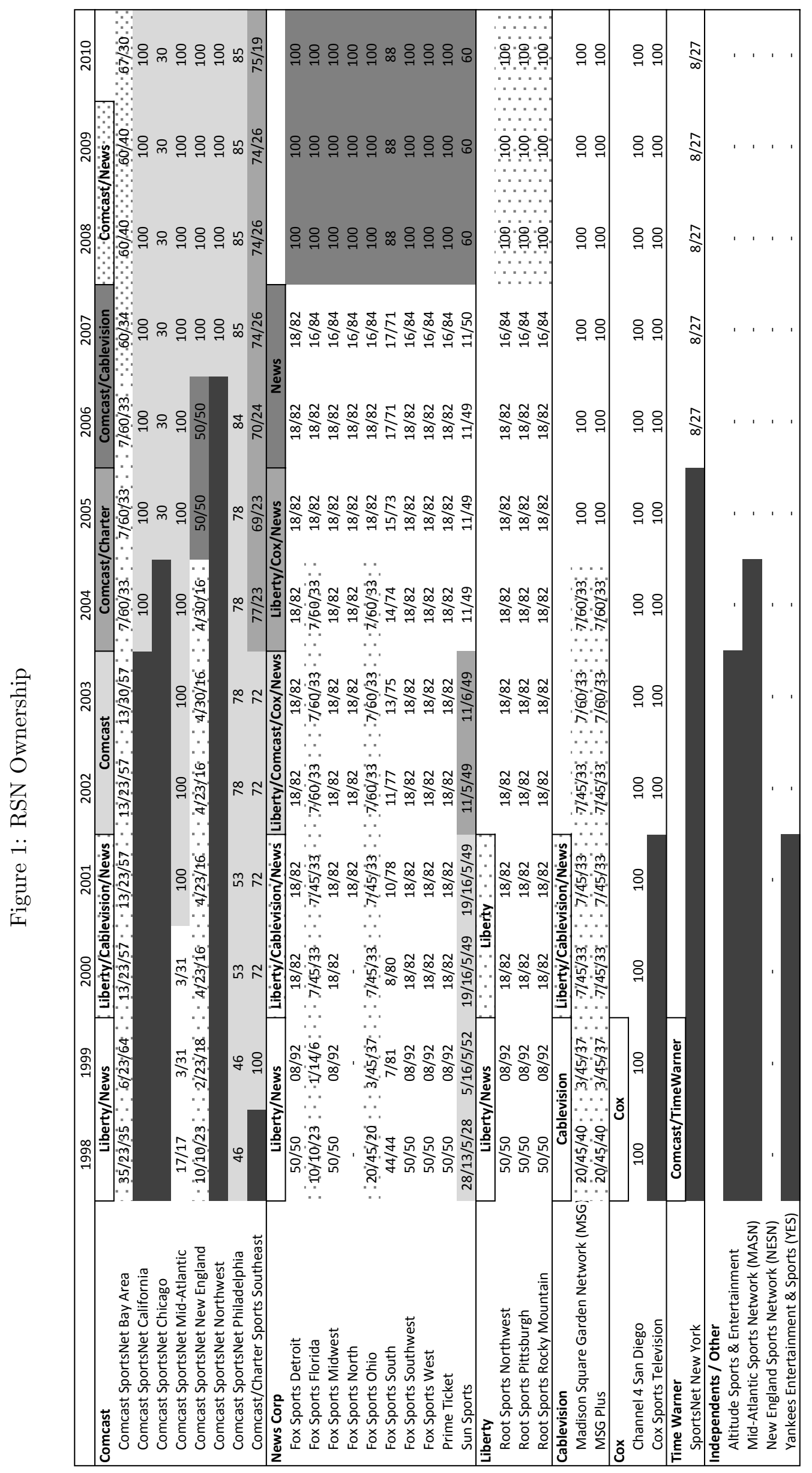

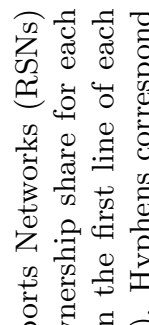

各 है।

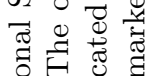

.000

車

昰

क्ष.

.

过星

สี

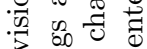

过寻焉

눙 $ᄋ$

曹

चี

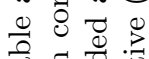

近 胥

品 者

읍 可.

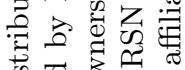

氜

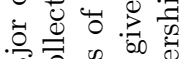

శ

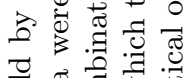

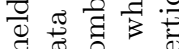

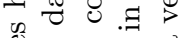

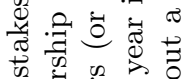

की

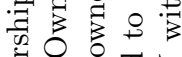

范范学

○。․ำ

.

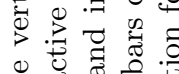

西 0

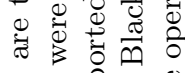

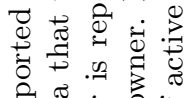

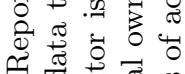

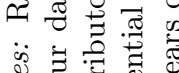

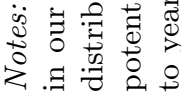


rules the Commission established in 2010 (FCC, 2012). The new case-by-case rules explicitly include a (rebuttable) presumption that exclusive deals between RSNs and their affiliated distributors are unfair. During our sample period (2000-2010), most integrated RSNs outside of loophole markets had agreements to be carried by all MVPDs. However, even though PARs were in effect, there were a few instances in which a cable-owned RSN was not carried by satellite distributors: e.g., in 2007, Comcast Sports Northwest, Comcast/Charter Sports Southeast, and Cox Sports Television were not broadcast on satellite distributors.

\subsection{Data}

We collect a wide variety of data to analyze the effects of vertical integration. We have three categories of data: (1) downstream prices, quantities, and characteristics of cable and satellite bundles, (2) channel viewership data, and (3) channel affiliate fees and advertising revenues. We briefly describe each in turn.

\subsubsection{Downstream Prices, Quantities, and Characteristics}

We combine data from multiple sources to construct downstream prices, quantities, and characteristics. Our foundational dataset is the Nielsen FOCUS database. For each cable system, it provides the set of channels offered (i.e., the channel "lineup"), the number of homes passed, the total number of subscribers (to any bundle of channels), the owner of the system, and the zip codes served. We use the years 2000 to 2010 . We restrict our analysis to system-years in which the system faced no direct competition from another cable distributor. ${ }^{13}$ We construct market shares by combining the number of subscribers reported by FOCUS (divided by the number of households in a market, obtained from 2000 and 2010 Census zip code data) with individual-level survey data from household survey firms Mediamark Research \& Intelligence (MRI) and Simmons, using MRI data for 2000 to 2007, and Simmons for 2008 to 2010. Specifically, if a system-year had at least 40 survey respondents, we use the average of the market share from the FOCUS data and the cable market share among the survey respondents; otherwise we use only the FOCUS data. We eliminate any system-year for which we had less than 40 individual-level survey respondents in the MRI/Simmons data and the FOCUS subscriber data were not updated from the previous year. We use the remaining system-years to construct our markets.

For our analysis, we define a market for each year to be a set of zip codes served by a single cable system and, by construction, both satellite distributors. For cable systems, we aggregate over bundles within a system, focusing on total system subscribers. Our demand model is therefore a distributor choice model, rather than a bundle choice model. ${ }^{14}$ We construct satellite shares within

\footnotetext{
${ }^{13}$ In our analysis, we focus only on markets in which there is a single cable and no other wireline (cable or telephone) distributor. We do so because when a system faces competition from another wireline distributor, we do not know the number of subscribers in the areas where the system faced competition relative to the areas where it did not. A second wireline distributor is present in $6 \%$ of all system-year observations. Our maintained assumption is that the omission of these markets does not affect the validity of our identifying assumptions or interpretation of results.

${ }^{14}$ The FOCUS data only report total subsscribers to the system, and our subscriber data are not rich enough to
} 
each of our markets for DirecTV and Dish Network from the MRI/Simmons survey data. ${ }^{15}$ We use historical channel offerings and prices for DirecTV and Dish Network collected via the Internet Archive (archive.org). Satellite bundles are assumed to vary across markets only in the set of RSNs carried. We assume that an RSN is carried by a satellite distributor in a given market if we observe that the satellite distributor carries that channel in any market, and the RSN is "relevant" in that market. We define an RSN to be relevant in a Nielson Designated Market Area (DMA) — and, hence, in all markets within that DMA - if, across all cable systems within that DMA at least 30 percent of the teams carried by the RSN are not "blacked out." 16 During our sample period, the average household subscribing to a cable distributor received $1.8 \mathrm{RSNs}$, and $80 \%$ of our markets have one or two relevant RSNs that are available.

We combine multiple sources of information on cable television prices. Systems regularly post prices on their websites and these websites are often saved in the Internet Archive. Following industry practice, we refer to the set of channels offered at a given (incremental) price as a tier of service and the combination of tiers chosen by households as the "bundle" that they buy. ${ }^{17}$ We use the price of the expanded basic bundle, the most popular bundle chosen by households and the bundle which typically contains all of the channels in our analysis. In addition to price information on systems' websites, we utilize newspaper reports of price changes which provide price information at the local cable system level. Some newspapers report this information every time cable prices change (typically yearly), providing valuable information about the history of price changes for a single (often large) system or geographic family of systems owned by the same distributor. Finally, cable systems typically have "rate cards" describing their current tiers, channels, and prices which they use for marketing or to inform customers of changes in these offerings; they were used when able to be found online. We searched the Internet for all such information about cable prices and linked by hand the information obtained to FOCUS systems based on the distributor, principal geographic region served, and other regions served as reported in the newspaper or listed on the rate card. For system-years where we do not find a price from websites, rate sheets, or newspapers, we link to the TNS Bill Harvesting database. The TNS data are individual-level bills for cable service which report the company providing the service, the household's expenditure, and their zip code. For a given system-year, we use the mean expenditure for subscribers to that system if the data contain at least 5 bills. ${ }^{18}$ These data also provide the level of any tax on cable and satellite

estimate bundle-specific quantities.

${ }^{15}$ We use a weighted average of state- and market-level satellite market shares, both calculated from the individuallevel MRI/Simmons data. If we have between 1-19 market-level observations, we weight the market-level share by 0.75 (and the state-level share by 0.25 ). If we have 20 or more market-level observations, we weight market-level shares by 0.90 . We dropped any constructed market whose total market share exceeded one or which, in the survey data, had a zero market share for one of the satellite distributors (which happens naturally due to sampling error).

${ }^{16}$ DMAs are mutually exclusive and exhaustive definitions of television markets created by Nielsen and used for the purchase of advertising time. Black-out rules are restrictions imposed by sports leagues that prevent broadcasts of a team's games in certain local markets. We use black-out information at the sport-team-zip-code level collected from MLB, NBA, and NHL websites in 2014 to determine whether a team is blacked-out in a given market.

${ }^{17}$ For example, the expanded basic bundle consists of the limited basic tier and the expanded basic tier.

${ }^{18}$ We only use bills which clearly delineate video programming costs (i.e., that separate it out from other bundled services such as internet and phone), and use the average of a system's revenue (excluding pay-per-view or one-time 
television services; we use state satellite taxes, which vary over time, as an instrumental variable for price in our demand estimation.

Table A.1 reports the average price, market share, and number of cable, RSN, and total channels offered across markets and years in our estimation dataset. We use 11 years of data, comprising almost 7,000 market-years, with an average coverage of 39.7 million (over $35 \%$ of) U.S. households per year. ${ }^{19}$ Average prices are quite similar across distributors, whether on an unweighted basis or weighted by the number of households in the market. The satellite distributors generally offer more channels on their Expanded Basic service than the local cable system, but a similar number of RSNs.

Finally, we derive MVPD margins for Comcast, DirecTV, and Dish from their 2007 10K reports; we use these as moments in our structural estimation. ${ }^{20}$

\subsubsection{Viewership}

We estimate demand using both bundle purchase and viewing data. We have two types of viewing data. One type provides information at the individual household level, and the other reports aggregate viewing decisions at the level of the DMA.

Individual household viewing data comes from the MRI and Simmons datasets described in the previous subsection. Our MRI data report the number of hours watched for each of the sampled households of 96 national channels from 2000 to 2007, while our Simmons data report the same information for 99 national channels between 2008 and 2010. Our aggregate ratings data come from Nielsen, which provides the percentage of households in a DMA watching a given program on a given channel at a given time. Reported is the average rating for each of between 63 and 100 channels, of which 18 to 29 are RSNs, depending on the year, in each of the 44 to 56 largest DMAs between 2000 and 2010 .

Tables A.2 and A.3 report summary statistics for our viewing data. Table A.2 reports, for each of our sources of viewing data, the mean rating for each of the 38 (non-RSN) national channels included in our econometric analysis. ${ }^{21}$ For example, the average rating for the ABC Family Channel in the Nielsen data across the 747 DMA-years for which the information was recorded is 0.418 percentage points. This suggests that a household selected at random in one of these years

charges) to construct prices.

${ }^{19}$ While we observe the complete population of channel lineups, incomplete reporting of subscriber information in the FOCUS dataset and the inability to collect cable prices in some markets prevents us from constructing the information we need in every U.S. cable market.

${ }^{20}$ We compute Comcast margins using video, advertising, and franchise fee revenues; programming expenses; and sales, general, and administrative (SG\&A) expenses multiplied by both the video revenue share of total revenues (to proportionately allocate expenses across Comcast's other businesses) and the share of SG\&A expenses that are subscriber acquisition and retention related (computed from DirecTV's reports). We compute DirecTV margins using total revenues; and programming, subscriber acquisition, upgrade, and retention expenses. We compute Dish margins using total revenues; subscriber acquisition costs; and the share of subscriber related expenses multiplied by the share of non-SG\&A costs (programming and service expenses) that are programming related (computed from DirecTV's reports). The computed values are $\{.539, .396, .413\}$.

${ }^{21}$ The 38 national channels represent the top 36 channels by viewership which have ratings data for each year, plus two smaller channels with sports related content (ESPN Classic and Golf Channel). 
and DMAs would be watching the ABC Family Channel with probability 0.418 percent. While small, this is above average for cable networks. Similarly, Table A.3 reports the average ratings for RSNs; for example, CSN Bay Area's average viewership is 0.41 percent. For RSN viewership, we have additional information - also reported in Table A.3 - about the average RSN rating by type of distributor (i.e., cable or each satellite operator).

Our household-level data provide further details about average viewing of national channels which are summarized in the remaining columns of Table A.2. The last column reports the share of households on average across DMAs and years that report any viewing of that channel. As noted in Crawford and Yurukoglu (2012), this provides valuable information about whether a household has any interest in a channel that we will use to inform the estimated distribution of preferences for channels across households.

\subsubsection{Average Affiliate Fees and Advertising Revenues}

As described earlier, affiliate fees are the monthly per subscriber charges paid by distributors to content providers for the ability to distribute the channel. SNL Kagan maintains a database with aggregate information about individual cable television networks, both nationally-distributed networks like CNN and ESPN as well as RSNs like the family of Comcast and Fox networks. For many networks, we use information about the average affiliate fee paid by cable and satellite MVPDs to each such network. Table A.2 reports average affiliate fees for each of the 38 national cable channels that we include in our analysis and Table A.3 reports the same information for each of the RSNs in our analysis. The average affiliate fee in our data for the national channels is $\$ 0.30$ per subscriber per month, while it is $\$ 1.64$ for our RSNs.

Per subscriber advertising rates are determined for each channel by dividing total advertising revenues by total subscribers (both provided by SNL Kagan).

\section{Model}

In this section, we present an industry model that predicts: (i) household viewership of channels; (ii) household demand for multichannel television services; (iii) prices and programming bundles that are offered by distributors; and (iv) negotiated distributor-channel specific affiliate fees. One key output from the specification and estimation of our model is the impact on viewership and demand of adding or removing channels from a bundle. This in turn informs the degree to which firms internalize the profits of integrated units when making strategic decisions, and the incentives of an integrated RSN to provide or withhold access to its content from rival distributors.

\subsection{Overview}

We index consumer households by $i$, markets by $m$, and time periods by $t$. There are a set of "downstream" multichannel video programming distributors (MVPDs) $\mathcal{F}_{t}$ and "upstream" channels $\mathcal{C}_{t}$ active in each period $t$. The set of MVPDs active in a given market-period is denoted $\mathcal{F}_{m t}$. We 
will assume that each such MVPD $f \in \mathcal{F}_{m t}$ offers a single bundle of channels $\mathcal{B}_{f m t} \subseteq \mathcal{C}_{t}$ in market $m$ and period $t$, where a household subscribing to this bundle pays a price $p_{f m t}$ and has access to all channels $c \in \mathcal{B}_{f m t}{ }^{22}$ Since we assume that distributors offer only one bundle, $f$ denotes both the distributor and the bundle it offers for a given market-period.

We assume that in each period $t$ (a year in our empirical work), decisions are made according to the following timing: in stage $\mathbf{1}$ channels and distributors bargain bilaterally to decide affiliate fees, and distributors set prices and make carriage decisions for each market in which they operate; in stage 2 households choose which MVPD, if any, to subscribe to in their market; and in stage 3 households view television channels. ${ }^{23}$ We now provide details of each stage and further assumptions, proceeding in reverse order of timing.

\subsection{Stage 3: Household Viewing}

We assume that households solve a time allocation problem to determine viewership. In particular, household $i$ in market $m$ and period $t$ subscribing to MVPD $f \in \mathcal{F}_{m t}$ allocates its time $\boldsymbol{w}_{\text {ift }} \equiv$ $\left\{w_{i f c t}\right\}_{c \in \mathcal{B}_{f m t} \cup\{0\}}$, where $w_{i f c t}$ is the time spent watching channel $c$ (or devoted to non-television activities if $c=0$ ), to solve:

$$
\begin{aligned}
\max _{\boldsymbol{w}_{i f t}} v_{i f t}\left(\boldsymbol{w}_{i f t}\right)= & \sum_{c \in \mathcal{B}_{f m t} \cup\{0\}} \frac{\gamma_{i c t}}{1-\nu_{c}}\left(w_{i f c t}\right)^{1-\nu_{c}} \\
\text { s.t. } \quad: \quad & w_{i f c t} \geq 0 \forall c, \\
& \sum_{c \in \mathcal{B}_{f m t} \cup\{0\}} w_{i f c t} \leq T .
\end{aligned}
$$

Parameters $\gamma_{i c t}$ and $\nu_{c} \in[0,1)$ govern consumer tastes for each channel $c$, where $\gamma_{i c t}$ sets the level of marginal utility of household $i$ from the first instant of watching the channel, and $\nu_{c}$ controls how fast this marginal utility decays with additional viewing. The parameter $T$ represents the total time available to the household. We restrict $\nu_{c}$ to be equal for all non-sport channels and the outside-option, and equal for all sports channels (which include RSNs); i.e., $\nu_{c}=\nu^{S}$ if $c$ is a sports channel, and $\nu_{c}=\nu^{N S}$ otherwise. ${ }^{24}$ We parameterize $\gamma_{i c t}$ as a function of channel-specific

\footnotetext{
${ }^{22}$ In the previous section, we explained why the data only permit us to look at demand for the most popular (expanded basic) bundle offered by each distributor in each market. We do not model distributor price discrimination at the market level (e.g., by offering multiple channel bundles or à la carte add-ons); see Crawford and Yurukoglu (2012) for further discussion. As modeled here, vertical integration does not affect how distributors are allowed to price, nor does it affect the ability of channels to price discriminate among distributors (who are charged a distributor-specific affiliate fee and cannot resell access to the channel; see also footnote 35).

${ }^{23}$ Stages 2 and 3 of our model describe a discrete-continuous choice model of consumer behavior over distributors and viewership (cf. Dubin and McFadden, 1984; Hanemann, 1984).

${ }^{24}$ Allowing for this parameter to differ between sports and non-sports channels is motivated by the observation that sports channels receive higher affiliate fees than national channels for the same viewership ratings; we discuss this further in Section 4.1.2. Our viewership model is equivalent to the Cobb-Douglas model used in Crawford and Yurukoglu (2012) if $\nu_{c} \rightarrow 1$ for all $c$.
} 
parameters $\boldsymbol{\rho}_{c} \equiv\left\{\rho_{c}^{0}, \rho_{c}^{1}\right\}$ as follows:

$$
\gamma_{i c t}= \begin{cases}\tilde{\gamma}_{i c t} & \text { with probability } \rho_{c}^{0}, \text { where } \tilde{\gamma}_{i c t} \sim \operatorname{Exponential}\left(\rho_{c}^{1}\right) \quad \forall c, t . \\ 0 & \text { with probability } 1-\rho_{c}^{0}\end{cases}
$$

For RSNs, we scale $\tilde{\gamma}_{i c t}$ by $\exp \left(\gamma^{b} b_{i c t}+\gamma^{d} d_{i c}\right)$, where $b_{i c t} \in[0,1]$ represents the fraction of teams carried on RSN $c$ that are "blacked-out" (i.e., unable to have games televised in household $i$ 's market), and $d_{i c}$ is the average distance from household $i$ to the stadiums of the teams shown on RSN $c$ (measured in thousands of miles). ${ }^{25}$ These terms allow for households to value an RSN differentially if the household cannot watch some of the carried sport teams, or if the household lives further away from the carried teams' stadiums.

\subsection{Stage 2: Household Distributor Choice}

Each period, household $i$ considers characteristics of the bundle offered by each MVPD $f \in \mathcal{F}_{m t}-$ including the utility obtained from watching channels in the bundle and its price - when determining which distributor, if any, to subscribe to. We specify household $i$ 's utility conditional on subscribing to $f$ as:

$$
u_{i f t}=\beta^{v} v_{i f t}^{*}+\boldsymbol{\beta}^{x} \boldsymbol{x}_{f t}+\beta_{i f}^{s a t}+\alpha p_{f t}+\xi_{f t}+\varepsilon_{i f t}
$$

where $v_{i f t}^{*}$, referred to as a consumer's viewership utility for the bundle offered by $f$, is the optimized value from the time allocation problem in (1), $\boldsymbol{x}_{f t}$ are firm-state and year dummy variables, $p_{f t}$ is the per month price (including any taxes), and $\xi_{f t}$ is a scalar unobservable demand shock for the bundle. Each consumer has a random preference for each satellite distributor, $\beta_{i f}^{\text {sat }}$, that is drawn from an independent exponential distribution with parameter $\rho_{f}^{\text {sat }}$; we assume that $\beta_{i f}^{\text {sat }}=0$ if $f$ is a cable distributor. ${ }^{26}$ We assume that the utility of the outside option of no bundle is normalized to $u_{i 0 t}=\varepsilon_{i 0 t}$, that $\varepsilon_{i t} \equiv\left\{\epsilon_{i f t}\right\}_{\forall f}$ is distributed Type I extreme value, and that each household chooses the bundle with the highest value of $u_{i f t} .{ }^{27}$

The probability that household $i$ chooses distributor $f$ in market $m$ is obtained by integrating over $\varepsilon_{i t}$ for each household:

$$
s_{i f m t}=\frac{\exp \left(\beta^{v} v_{i f t}^{*}+\boldsymbol{\beta}^{x} \boldsymbol{x}_{f t}+\beta_{i f}^{s a t}+\alpha p_{f t}+\xi_{f t}\right)}{1+\sum_{g \in \mathcal{F}_{m t}} \exp \left(\beta^{v} v_{i g t}^{*}+\boldsymbol{\beta}^{x} \boldsymbol{x}_{g t}+\beta_{i g}^{s a t}+\alpha p_{g t}+\xi_{g t}\right)} .
$$

\footnotetext{
${ }^{25} \mathrm{RSNs}$ may be carried by systems outside of a team's local area for at least two reasons. First, an RSN may broadcast games from different sport leagues with different black-out restrictions. For example, CSN Chicago is carried on systems in Indianapolis even though Chicago Bulls games that the RSN broadcasts are blacked-out (Indianapolis is Pacer's territory), as the RSN also broadcasts Chicago Cubs games (which are considered in-market for Indianapolis). The second reason is that RSNs also broadcast programming not subject to black-out restrictions, which include other sports (e.g., racing, boxing, poker) in addition to sports news and specialized programming. We focus only on blackout restrictions for MLB, NBA, and NHL teams. We ignore the NFL in our analysis since its games have only been aired by national channels since the 1960s (CBS, NBC, Fox, and ESPN currently own its television rights).

${ }^{26}$ As we discuss in the next section, allowing for heterogeneity in preferences for satellite bundles assists our model in matching observed distributor price-cost margins.

${ }^{27}$ Our normalization allows for variation in the quality of the outside option (which includes local antenna reception for television signals) across markets and time due to our inclusion of firm-state and year dummy variables.
} 
The total market share for distributor $f$ (in market $m$ at time $t$ ) is then $s_{f m t} \equiv \int s_{i f m t} d H_{m t}(i)$, where $H_{m t}(i)$ is the joint distribution of household random coefficients $(\boldsymbol{\gamma}, \boldsymbol{\beta})$ in the market, and the demand for distributor $f$ is $D_{f m t} \equiv N_{m t} s_{f m t}$, where $N_{m t}$ is the number of television households in the market.

\subsection{Stage 1: Distributor Pricing, Carriage, and Affiliate Fee Bargaining}

In Stage 1, all MVPDs and channels bargain over affiliate fees $\boldsymbol{\tau}_{t} \equiv\left\{\tau_{f c t}\right\}_{\forall f, c}$, where $\tau_{f c t}$ represents the period $t$ fee that distributor $f$ pays the owner of channel $c$ for each of $f$ 's household subscribers. Simultaneously, each distributor chooses the prices and channel composition of its bundle in every market in which it operates. ${ }^{28}$ That is, we assume that bargaining occurs simultaneously with the determination of distributor pricing and carriage decisions; we provide further discussion of this assumption in Section 3.4.3.

We assume that affiliate fees, bundle prices, and bundle compositions are optimal with respect to one another in equilibrium. We now discuss these optimality conditions in more detail, considering first distributors' pricing and carriage decisions (given the affiliate fee bargaining outcome), and then affiliate fee bargaining (given distributor pricing and carriage).

\subsubsection{Stage 1a. Distributor Pricing and Carriage}

Each period, every MVPD $f \in \mathcal{F}_{t}$ chooses prices and the channels offered in each of its bundles $\left\{p_{f m t}, \mathcal{B}_{f m t}\right\}_{\forall m: f \in \mathcal{F}_{m t}}$ to maximize its profits given negotiated affiliate fees $\boldsymbol{\tau}_{t}$. Profits for $f$ across all markets are:

$$
\Pi_{f t}^{M}\left(\left\{\mathcal{B}_{m t}\right\}_{m},\left\{\boldsymbol{p}_{m t}\right\}_{m}, \boldsymbol{\tau}_{t} ; \mu\right)=\sum_{m: f \in \mathcal{F}_{m t}} \Pi_{f m t}^{M}\left(\mathcal{B}_{m t}, \boldsymbol{p}_{m t}, \boldsymbol{\tau}_{t} ; \mu\right)
$$

where:

$$
\Pi_{f m t}^{M}\left(\mathcal{B}_{m t}, \boldsymbol{p}_{m t}, \boldsymbol{\tau}_{t} ; \mu\right)=D_{f m t} \times\left(p_{f m t}^{\mathrm{pre}-\mathrm{tax}}-m c_{f m t}\right)+\mu \times\left(\sum_{g \in \mathcal{F}_{m t}} \sum_{c \in \mathcal{B}_{g m t}} O_{f c t}^{M} \times D_{g m t} \times\left(\tau_{g c t}+a_{c t}\right)\right)
$$

(and, as in the rest of this section, $\mathcal{F}_{m t}$ also includes $f$ ). In expression (4), we denote by $\mathcal{B}_{m t} \equiv$ $\left\{\mathcal{B}_{f m t}\right\}_{f \in \mathcal{F}_{m t}}$ and $\boldsymbol{p}_{m t} \equiv\left\{p_{f m t}\right\}_{f \in \mathcal{F}_{m t}}$ the set of channels and associated prices offered in market $m$, and by $a_{c t}$ the expected per-subscriber advertising revenue obtained by channel $c$ from bundles that carry $c$. Firm revenues are derived from pre-tax prices, $p_{f m t}^{\text {pre-tax }} \equiv p_{f m t} /\left(1+\operatorname{tax}_{f m t}\right)$, which are a function of market-specific cable or satellite tax rates that are known and assumed to be determined exogenously. The term $O_{f c t}^{M}$ is a function of MVPD $f$ 's ownership share of channel $c$ at time $t$; we refer to $f$ and $c$ as being integrated if $O_{f c t}^{M}>0$, with full integration equivalent to

\footnotetext{
${ }^{28}$ A given cable distributor $f$ often operates in many markets, and is choosing its price and set of channels to offer in each of these markets. Satellite distributors choose a single national price and channel bundle, with the only potential variation across DMAs being the set of RSNs that are carried.
} 
$O_{f c t}^{M}=1 .{ }^{29}$ The parameter $\mu$, which we will estimate, captures the extent to which a downstream MVPD $f$ internalizes upstream affiliate fees and advertising revenues from its integrated channels.

The first component of (4), an MVPD's profit function in a given market $m$, is standard: each bundle has a price and a marginal cost $\left(m c_{f m t}\right)$ that determine its margin, and this is multipled by its demand. We assume that each MVPD $f$ 's marginal cost in market $m$ can be decomposed into the sum of the per subscriber fees that $f$ must pay to the various channels in its marketbundle, and bundle-specific non-channel-related marginal cost, denoted by $\kappa_{f m t}$ : i.e., $m c_{f m t} \equiv$ $\sum_{c \in \mathcal{B}_{f m t}} \tau_{f c t}+\kappa_{f m t} .{ }^{30}$ The second component of the profit function is non-standard, and represents the degree to which a vertically integrated downstream unit values the profits that accrue to its upstream (i.e., channel) units. These terms include per subscriber fees $\left(\tau_{g c t}\right)$ and advertising revenues $\left(a_{c t}\right)$ that accrue to integrated upstream channels from MVPD $f$ 's own viewers as well as from viewers of other distributors $g \neq f$, and are multiplied by the ownership share variables $O_{f c t}^{M}$ and parameter $\mu .{ }^{31}$ In the absence of any intra-firm frictions, $\mu$ would equal one, implying that the downstream unit of an integrated firm perfectly internalizes its (fully) integrated upstream units' profits, and its strategic decisions maximize total firm profit. The parameter $\mu$ could also be less than one, potentially representing divisionalization that could arise from ignorance, poor management, optimal compensation under informational frictions, or any other conflict between managers of different divisions within the same firm. By estimating $\mu$ we seek to uncover the extent to which such internalization actually occurs in our setting.

Cable Pricing and Carriage. We will leverage necessary conditions on the optimality of MVPD pricing and carriage decisions in our estimation. Differentiating (4) with respect to $p_{f m t}$ (and dividing by market size) yields the following pricing first-order condition:

$$
\frac{\partial \Pi_{f m t}^{M}}{\partial p_{f m t}}=\frac{s_{f m t}}{1+\operatorname{tax}_{f m t}}+\left(p_{f m t}^{\text {pre-tax }}-m c_{f m t}\right) \frac{\partial s_{f m t}}{\partial p_{f m t}}+\mu \times\left(\sum_{g \in \mathcal{F}_{m t}} \sum_{c \in \mathcal{B}_{g m t}} O_{f c t}^{M} \frac{\partial s_{g m t}}{\partial p_{f m t}}\left(\tau_{g c t}+a_{c t}\right)\right)=0 .
$$

With regard to carriage, a cable distributor's optimal decision for an RSN is indeterminate when no deal is reached between the distributor and that RSN: i.e., whether or not the distributor would carry the RSN on a subset of its systems in the event the RSN were available is irrelevant when the RSN is not available to the distributor at all. In our estimation, we will therefore make use of bundle optimality conditions for cable operators only for channels with which they have an agreement. Thus, we assume that the set of channels that are offered by each cable MVPD $f$ in

\footnotetext{
${ }^{29}$ In Appendix C.1, we detail the construction of this and our other ownership variables (introduced later). For our analysis, we restrict $O_{f c t}^{M}=0$ if $c$ is not an RSN.

${ }^{30}$ Non-channel related costs include technical service, labor, gasoline, and equipment costs that are incurred on a per subscriber basis.

${ }^{31}$ We omit portions of integrated channels' profits that are not affected by $f$ 's pricing and carriage decisions, as they do not affect the analysis. We also assume that channel $c$ 's per subscriber advertising revenues in market $m$ do not vary across MVPDs, and that channel $c$ 's marginal costs per subscriber are zero.
} 
each market $m$ satisfies:

$$
\mathcal{B}_{f m t}=\arg \max _{\mathcal{B}_{f} \subseteq \mathcal{A}_{f t}} \Pi_{f m t}^{M}\left(\left\{\mathcal{B}_{f}, \mathcal{B}_{-f, m t}\right\}, \boldsymbol{p}_{m t}, \boldsymbol{\tau}_{t} ; \mu\right)
$$

where $\mathcal{A}_{f t} \subseteq \mathcal{C}_{t}$ is the set of channels available to MVPD $f$ : i.e., the set of channels for which $f$ has reached an agreement.

Satellite Pricing and Carriage. If instead distributor $f$ is a satellite MVPD (DirecTV or Dish), we assume that the distributor sets a single national price and bundle. This national satellite price satisfies a similar optimality condition to (5) above. We assume that the bundle offered by a satellite MVPD in any given market may differ from the national bundle only in the set of RSN channels that are offered. In addition, we assume that satellite distributors adopt the strategy of carrying any channel for which they have negotiated a deal (intuitively, since any deal that is reached should make carriage profitable). ${ }^{32}$

\subsubsection{Stage 1b: Bargaining over affiliate fees}

Before describing how affiliate fees are determined, we specify the profits that each channel $c$ contemplates when bargaining with MVPD $f$. We assume that if $f$ and $c$ are integrated (i.e., $\left.O_{f c t}^{M}>0\right), c$ 's profits in market $m$ are:

$$
\begin{aligned}
\Pi_{c m t}^{C}\left(\mathcal{B}_{m t}, \boldsymbol{p}_{m t}, \boldsymbol{\tau}_{t} ; \mu\right) & =\sum_{g \in \mathcal{F}_{m t}: c \in \mathcal{B}_{g m t}} D_{g m t} \times\left(\tau_{g c t}+a_{c t}\right) \ldots \\
+\mu & \sum_{g \in \mathcal{F}_{m t}} D_{g m t} \times\left(O_{g c t}^{C} \times\left(p_{g m t}^{\text {pre-tax }}-m c_{g m t}\right)+\sum_{d \in \mathcal{B}_{g m t} \backslash c} O_{c d t}^{C C} \times\left(\tau_{g d t}+a_{g d t}\right)\right)
\end{aligned}
$$

However, if $f$ and $c$ are not integrated, $c$ 's profits in $m$ are:

$$
\begin{aligned}
\Pi_{c m t}^{C}\left(\mathcal{B}_{m t}, \boldsymbol{p}_{m t}, \boldsymbol{\tau}_{t} ; \mu, \lambda_{R}\right) & =\sum_{g \in \mathcal{F}_{m t}: c \in \mathcal{B}_{g m t}} D_{g m t} \times\left(\tau_{g c t}+a_{c t}\right) \ldots \\
& +\mu \sum_{g \in \mathcal{F}_{m t}} D_{g m t} \times\left(\lambda_{R} \times O_{g c t}^{C} \times\left(p_{g m t}^{\text {pre-tax }}-m c_{g m t}\right)+\sum_{d \in \mathcal{B}_{g m t} \backslash c} O_{c d t}^{C C} \times\left(\tau_{g d t}+a_{g d t}\right)\right) .
\end{aligned}
$$

In both (7) and (8), the first lines represent affiliate fees and advertising revenues that channel $c$ obtains from each bundle on which the channel is available in market $m$, and the second lines incorporate channel c's potential profits from its integrated downstream MVPDs (based on $O_{g c t}^{C}$, a function of the ownership share of $c$ held by MVPD $g$ ), as well as profits from other integrated channels $d$ of channel $c$ (which depend on $O_{c d t}^{C C}$, which is a function of the common ownership shares of channels $c$ and $d$; see Appendix C.1 for further details). ${ }^{33}$ These integrated profits are in

\footnotetext{
${ }^{32}$ For RSNs, we make this assumption only in the RSN's relevant markets.

${ }^{33}$ In 2007, $4 \%$ of markets in our sample have two RSNs that are relevant and share a common owner; none have three or more relevant RSNs that share a common owner.
} 


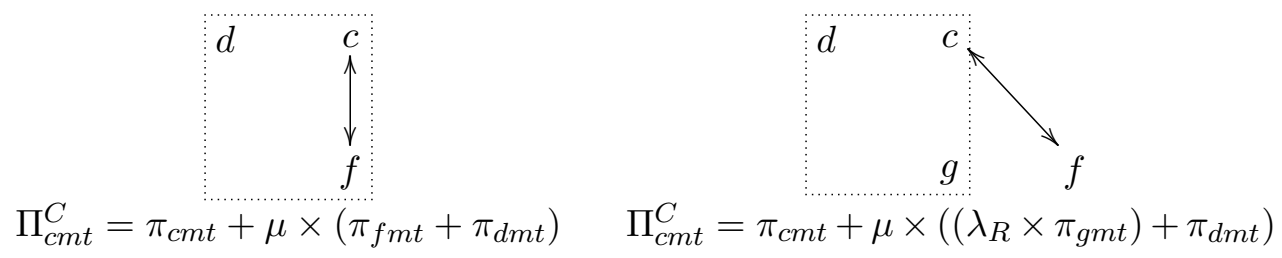

(a) Internal Bargaining.

(b) Bargaining with Rival MVPD.

Figure 2: Examples of $\Pi_{c m t}^{C}$ when $c$ bargains with MVPD $f$.

each case multiplied by $\mu$, the parameter capturing the extent of within-firm internalization across divisions.

The one difference between (7) and (8) is that in the latter expression, which is relevant when an integrated RSN $c$ bargains with a non-integrated distributor $f$, any effects of the deal on downstream distributors integrated with $c$ are multiplied by the parameter $\lambda_{R} \geq 0$. This parameter - our "rival foreclosure" parameter - captures the extent to which channel $c$ considers the benefits of foreclosure (the denial of access to channel $c$ ) to its downstream division $g$ in its decision of whether to supply $f$. This benefit arises because non-supply lowers the quality of $f$ 's bundle, shifting demand to $c$ 's downstream division $g$. By including and estimating this parameter - rather than simply setting it equal to the theoretical value of 1 -we aim to estimate the extent to which foreclosure concerns actually motivate integrated RSNs' supply decisions to non-integrated downstream rivals.

In Figure 2, we provide an illustration of how channel c's perceived profits when bargaining with MVPD $f$ may change depending on whether or not it is integrated with $f$. In Figure 2a, the dashed square represents the fact that channel $c$ is fully integrated with MVPD $f$ (so that $O_{f c t}^{M}=O_{f c t}^{C}=1$ ) and another channel $d$; in this case, when bargaining with its integrated distributor $f$, channel $c$ will consider its own profits (denoted by $\pi_{c m t}$ ), consisting of affiliate fees and advertising revenues, as well as profits of $f$ and its integrated channel $d$ (denoted by $\pi_{f}$ and $\pi_{d}$ ), weighted by $\mu$ : i.e., $\Pi_{c m t}^{C}=\pi_{c m t}+\mu \times\left(\pi_{f m t}+\pi_{d m t}\right)$. We assume that $\pi_{f m t}$ includes $f$ 's subscription revenues net of its costs, whereas profits $\pi_{d m t}$ include $d$ 's affiliate fees and advertising revenues. In Figure 2b, channel $c$ is instead integrated with another MVPD $g$ (and still channel $d$ ); in this case, channel $c$ will consider its own profits $\pi_{c m t}$ when bargaining with $f$ (now a rival MVPD), as well as those of its integrated units $\pi_{g m t}$ and $\pi_{d m t}$, weighted by $\mu \times \lambda_{R}$ and $\mu$, respectively: i.e., $\Pi_{c m t}^{C}=\pi_{c m t}+\mu \times\left(\left(\lambda_{R} \times \pi_{g m t}\right)+\pi_{d m t}\right)$.

The parameter $\lambda_{R}$ (multiplied by $\mu$ ) thus captures the internalization of an integrated downstream MVPD's profits when an integrated channel bargains with another non-integrated distributor. In the case considered in Figure $2 \mathrm{~b}$, a higher value of $\lambda_{R}$ increases channel $c$ 's desire to raise downstream profits of its fully integrated distributor $g$, and lowers $c$ 's gains from trade when bargaining with the non-integrated rival MVPD $f$. This may lead to an increased affiliate fee $\left(\tau_{f c t}\right)$ for the rival distributor $f$. If the overall gains from trade are eliminated instead, it may lead to non-supply of channel $c$ to $f$ altogether. ${ }^{34}$

\footnotetext{
${ }^{34}$ When it does not lead to non-supply, a positive value of our rival foreclosure parameter $\lambda_{R}$ will lead to an increased
} 
Finally, when $c$ is only partially integrated with a distributor, the internalization parameter $\mu$ is multiplied by our ownership share variable in firms' perceived profits: e.g., in the case of external bargaining with distributor $f$ when $c$ is partially integrated with distributor $g, c$ 's perceived profits are $\Pi_{c m t}^{C}=\pi_{c m t}+\mu \times O_{g c t}^{C} \times\left(\left(\lambda_{R} \times \pi_{g m t}\right)+\pi_{d m t}\right)$.

Bargaining. We assume that, given channel $c$ is carried on some of MVPD $f$ 's systems, the affiliate fee $\tau_{f c t}$ between distributor $f$ and channel $c$ maximizes their respective bilateral Nash products given the expected negotiated affiliate fees of all other pairs and the expected prices and bundles for all distributors. In other words, affiliate fees $\tau_{t}$ satisfy:

$$
\begin{aligned}
\tau_{f c t}\left(\boldsymbol{\tau}_{-f c, t}, \mathcal{B}_{t}, \boldsymbol{p}_{t}\right)=\arg \max _{\tau_{f c t}} & {[\underbrace{\sum_{m \in \mathcal{M}_{f c t}}\left[\Delta_{f c} \Pi_{f m t}^{M}\left(\mathcal{B}_{m t}, \boldsymbol{p}_{m t},\left\{\tau_{f c t}, \boldsymbol{\tau}_{-f c, t}\right\} ; \mu\right)\right]}_{G F T_{f c t}^{M}\left(\tau_{f c t}, \cdot\right)}]^{\zeta_{f c t}} } \\
\times & {[\underbrace{\sum_{m \in \mathcal{M}_{f c t}}\left[\Delta_{f c} \Pi_{c m t}^{C}\left(\mathcal{B}_{m t}, \boldsymbol{p}_{m t},\left\{\tau_{f c t}, \boldsymbol{\tau}_{-f c, t}\right\} ; \mu, \lambda_{R}\right)\right]}_{G F T_{f c t}^{C}\left(\tau_{f c t}, \cdot\right)}]^{1-\zeta_{f c t}} \forall f, c \in \mathcal{A}_{f t}, }
\end{aligned}
$$

where $\mathcal{M}_{f c t} \equiv\left\{m: c \in \mathcal{B}_{f m t}\right\}$ denotes the set of markets in which $c$ is carried on $f$ 's bundle, $\zeta_{f c t} \in[0,1]$ represents a firm-channel-time specific Nash bargaining parameter, and:

$$
\begin{aligned}
& {\left[\Delta_{f c} \Pi_{f m t}^{M}\left(\mathcal{B}_{m t}, \cdot\right)\right] \equiv\left(\Pi_{f m t}^{M}\left(\mathcal{B}_{m t}, \cdot\right)-\Pi_{f m t}^{M}\left(\mathcal{B}_{m t} \backslash f c, \cdot\right)\right),} \\
& {\left[\Delta_{f c} \Pi_{c m t}^{C}\left(\mathcal{B}_{m t}, \cdot\right)\right] \equiv\left(\Pi_{c m t}^{C}\left(\mathcal{B}_{m t}, \cdot\right)-\Pi_{c m t}^{C}\left(\mathcal{B}_{m t} \backslash f c, \cdot\right)\right)}
\end{aligned}
$$

where we denote by $\mathcal{B}_{m t} \backslash f c$ the set of all bundles in $\mathcal{B}_{m t}$ with channel $c$ removed from bundle $f$. These last two terms represent the difference in either MVPD or channel profits in market $m$ if $f$ no longer carries channel $c$. We will refer to $G F T_{f c t}^{M}\left(\tau_{f c t}, \cdot\right)$ and $G F T_{f c t}^{C}\left(\tau_{f c t}, \cdot\right)$, which are the sums of these terms across all markets, as the gains from trade for MVPD $f$ and channel $c$ coming to an agreement with affiliate fee $\tau_{f c t}$. We assume that each MVPD and channel negotiate a single affiliate fee that applies to all markets. ${ }^{35}$

input fee for non-integrated downstream rivals by reducing c's gains from trade. This "raising rivals' costs" effect differs from that in Salop and Scheffman (1983) and Krattenmaker and Salop (1986): in those papers, the supplier has all the bargaining power and is motivated by the effect that raising its input price has on downstream prices to consumers. With our simultaneous timing, channel $c$ instead considers consumer prices as fixed when it bargains. Nonetheless, in equilibrium an increase in $c$ 's input fee can lead the non-integrated downstream distributor to raise its bundle price to consumers, just as the equilibrium downstream price increases in the double-marginalization example of Section 3.4.3 when the distributor's effective affiliate fee increases there. (Although in our main counterfactual specifications we assume that national satellite prices are held fixed, we also consider as an extension cases in which satellite bundle prices can adjust on a local level.)

${ }^{35}$ We rule out the possibility that RSNs are able to negotiate market-specific affiliate fees for each distributor (thereby engaging in a form of price discrimination across markets). Such richer pricing could reduce the degree of inefficient carriage decisions present in these markets, and thereby alter the welfare effects of vertical integration. To 
In Appendix C.1, we show that when $f$ and $c$ share at most one common owner (which is the case for all MVPD-channel pairs considered in our analysis), $O_{f c t}^{M}=O_{f c t}^{C} \equiv O_{f c t}$. When this holds, we can write the first-order condition of (9) for each channel $c$ bargaining with MVPD $f$ as:

$$
\left(1-\zeta_{f c t}\right) \times G F T_{f c t}^{M}\left(\tau_{f c t}, \cdot\right)=\zeta_{f c t} \times G F T_{f c t}^{C}\left(\tau_{f c t}, \cdot\right) \quad \forall f, c \in \mathcal{A}_{f t},
$$

which states that the equilibrium negotiated input fee $\tau_{f c t}$ between channel $c$ and distributor $f$ equalizes their (weighted) gains-from-trade. ${ }^{36}$

Alternatively, letting $\mu_{f c t} \equiv \mu \times O_{f c t}$, observe that $\operatorname{GFT}_{f c t}^{M}\left(\tau_{f c t}, \cdot\right)=\operatorname{GFT}_{f c t}^{M}(0, \cdot)-(1-$ $\left.\mu_{f c t}\right) \sum_{m \in \mathcal{M}_{f c t}} D_{f m t} \tau_{f c t}$ and $G F T_{f c t}^{C}\left(\tau_{f c t}, \cdot\right)=G F T_{f c t}^{C}(0, \cdot)+\left(1-\mu_{f c t}\right) \times \sum_{m \in \mathcal{M}_{f c t}} D_{f m t} \tau_{f c t}$, where we omit the arguments of $D_{f m t}$ for convenience. Thus, we can rewrite (10) as:

$$
\left[\left(1-\mu_{f c t}\right) \tau_{f c t}\right] \times \sum_{m \in \mathcal{M}_{f c t}} D_{f m t}=\left(1-\zeta_{f c t}\right) \times G F T_{f c t}^{M}(0, \cdot)-\zeta_{f c t} \times G F T_{f c t}^{C}(0, \cdot)
$$

which relates the "effective" total payments made by distributor $f$ to channel $c$, given by the left hand side of (11), to a weighted sum of the gains from trade due to agreement at $\tau_{f c t}=0$, given by the right hand side. The effective total payments nets out the $\mu_{f c t}$ fraction of $f$ 's affiliate fee payments to an integrated unit $c$ that are not considered by $f$ when making pricing, carriage, or bargaining decisions (see (4)). Intuitively, the more that $f$ gains from the relationship, the higher the total (effective) payment that is made; the more that $c$ gains from the relationship, the lower the total payment. If $f$ and $c$ 's Nash bargaining parameters were equal, then $\zeta_{f c t}=1 / 2$ and the total gain from trade would be split in half.

For estimation and our counterfactual simulations, we assume Nash bargaining parameters $\zeta_{f c t}=\zeta^{I}$ or $\zeta_{f c t}=\zeta^{E}$ depending on whether $c$ and $f$ are integrated $\left(O_{f c t}>0\right)$ and bargain internally $(\mathrm{I})$, or are non-integrated $\left(O_{f c t}=0\right)$ and bargain externally $(\mathrm{E})$.

Bargaining Example. Consider the case in which MVPD $f$ and channel $c$ are both nonintegrated entities that bargain with one another in period $t$. The negotiated affiliate fee $\tau_{f c t}$

our knowledge, however, such contracts are not widely employed in this industry.

${ }^{36}$ When $f$ and $c$ are bargaining with one another and $\mu_{f c t} \equiv \mu \times O_{f c t} \neq 1$ :

$$
\begin{aligned}
& \frac{\partial G F T_{f c t}^{M}(\cdot)}{\partial \tau_{f c t}}=\sum_{m} \frac{\partial \Pi_{f m t}^{M}}{\partial \tau_{f c t}}=-\left(1-\mu_{f c t}\right) \sum_{m \in \mathcal{M}_{f c t}} D_{f m t}, \\
& \frac{\partial G F T_{f c t}^{C}(\cdot)}{\partial \tau_{f c t}}=\sum_{m} \frac{\partial \Pi_{c m t}^{C}}{\partial \tau_{f c t}}=\left(1-\mu_{f c t}\right) \sum_{m \in \mathcal{M}_{f c t}} D_{f m t} ;
\end{aligned}
$$

thus $\partial G F T_{f c t}^{M} / \partial \tau_{f c t}=-\partial G F T_{f c t}^{C} / \partial \tau_{f c t}$ and (10) follows. The bargaining solution given by (9) is not defined if $\mu_{f c t}=1$; in this case, $f$ and $c$ perfectly internalize each other's profits when bargaining with one another, and the negotiated $\tau_{f c t}$ is indeterminate. 
that satisfies the Nash bargaining solution given by (11) solves:

$$
\begin{aligned}
\sum_{m \in \mathcal{M}_{f c t}} D_{f m t} \tau_{f c t}=\left(1-\zeta_{f c t}\right) & \underbrace{\sum_{m \in \mathcal{M}_{f c t}}\left(\left[\Delta_{f c} D_{f m t}\right]\left(p_{f m t}^{\mathrm{pre}-\mathrm{tax}}-m c_{f m t \backslash f c}\right)\right)}_{G F T_{f c t}^{M}(0, \cdot)} \\
& -\left(\zeta_{f c t}\right) \underbrace{\sum_{m \in \mathcal{M}_{f c t}}\left(D_{f m t} a_{c t}+\sum_{g \neq f: c \in \mathcal{B}_{g m t}}\left[\Delta_{f c} D_{g m t}\right]\left(\tau_{g c t}+a_{c t}\right)\right)}_{G F T_{f c t}^{C}(0, \cdot)},
\end{aligned}
$$

where $\left[\Delta_{f c} D_{g m t}\right] \equiv D_{g m t}\left(\mathcal{B}_{m t}, \cdot\right)-D_{g m t}\left(\mathcal{B}_{m t} \backslash f c, \cdot\right)$ denotes the change in firm $g$ 's demand in market $m$ and time $t$ if channel $c$ was removed from firm $f$ 's bundle, and $m c_{f m t \backslash f c} \equiv \sum_{d \in \mathcal{B}_{f m t} \backslash c} \tau_{f d t}+\kappa_{f m t}$. As before, the left hand side of (12) represents the total payment made by distributor $f$ to channel $c$. It is increasing in the additional profits (not including payments to $c$ ) that $f$ receives from the additional subscribers induced by the carriage of channel $c$ (given by the first line of the right hand side), decreasing in c's advertising revenues due to $f$ 's subscribers (represented by the terms $D_{f m t} a_{c t}$ ), and increasing in $c$ 's loss in profits from other distributors as a result of being carried on $f$ (as $\left[\Delta_{f c} D_{g m t}\right]<0$ for $g \neq f$ ). This last term, given by $\left[\Delta_{f c} D_{g m t}\right]\left(\tau_{g c t}+a_{c t}\right)$ summed across other distributors $g$, can be interpreted as an opportunity cost borne by channel $c$ from supplying distributor $f$, and relates the equilibrium affiliate fees that channel $c$ receives from all distributors to each other (Chen, 2001).

\subsubsection{Remarks on Timing and Bargaining}

Our bargaining solution assumes that each pair of distributors and channels agree upon a set of affiliate fees that maximize the Nash product of their gains from trade. It is motivated by the model put forth in Horn and Wolinsky (1988), and used by Crawford and Yurukoglu (2012) to model negotiations between MVPDs and channels. Other empirical papers that employ this concept, occasionally referred to as the "Nash-in-Nash" bargaining solution, include Draganska et al. (2010), Grennan (2013), Gowrisankaran et al. (2015), and Ho and Lee (2017). Collard-Wexler et al. (2015) provide a non-cooperative foundation for this particular bargaining solution based on a model of alternating offer bargaining in settings where agents negotiate fixed fee transfers and can engage in multilateral deviations. ${ }^{37}$

Our model also assumes that bargaining over affiliate fees happens simultaneously with distributors making carriage and pricing decisions. This assumption simplifies the estimation and computation of our model. For example, we leverage the simultaneity of bargaining and pricing in deriving (10), as there is no anticipated change in $p_{f m t}$ if $\tau_{f c t}$ changes. Formally, one can think of separate divisions of the distributor engaging in different functions or actions: e.g., a central

\footnotetext{
${ }^{37}$ Our model differs from Collard-Wexler et al. in that agents negotiate over linear fees, $\tau$. However, as (11) makes clear, the total equilibrium payment that is made between a channel and a distributor in our setting (given deals with other distributors) is equivalent to that when their bargaining is over a fixed fee.
} 
division bargains over affiliate fees, while local offices determine both pricing and carriage. ${ }^{38}$ An alternative timing assumption would be to assume that affiliate fees are first negotiated, and then distributor prices and bundles are chosen. This would adjust firms' perceptions of off-equilibrium path actions: e.g., when bargaining, firms would anticipate different bundle prices to be set immediately if off-equilibrium path affiliate fees or disagreement were realized. However, there may be reasons to believe that such a rapid response is unrealistic. Absent a fully specified dynamic model of firm bargaining and pricing, which is outside the scope of the current analysis, we believe the approach taken here to be a reasonable approximation.

Nonetheless, in equilibrium, all supply decisions must satisfy the conditions put forth in (5), (6) and (10): i.e., bundle prices and carriage are optimal with respect to equilibrium affiliate fees, and affiliate fees are negotiated while conditioning on equilibrium bundle prices and carriage. Hence, as the following example illustrates, when MVPD $f$ integrates with channel $c$, lower negotiated affiliate fees $\tau_{f c t}$ can still lead to a lower downstream price $p_{f m t}$.

Example: Integration, Internalization, and Double Marginalization. To illustrate both how our model leads to double marginalization and the effect of changes in our internalization parameter $\mu$, consider a simple setting with a single channel $c$ fully owned by a single MVPD $f$. Downstream demand is $D(p)$, while per subscriber costs are $m c_{f}$ for the MVPD (channel $c$ incurs no marginal costs). Given affiliate fee $\tau$, MVPD $f$ sets bundle price $p=\phi\left(m c_{f}+(1-\mu) \tau\right)$, where $\phi^{\prime}(\cdot)>0$. On the other hand, given a bundle price $p$, the gains from trade (at an affiliate fee of zero) for channel $c$ and MVPD $f$ are

$$
\begin{aligned}
\operatorname{GFT}_{c}^{C}(0, \cdot) & =0+\mu \times\left(p-m c_{f}\right) D(p)=\mu \times\left(p-m c_{f}\right) D(p) \\
\operatorname{GFT}_{f}^{M}(0, \cdot) & =\left(p-m c_{f}\right) D(p)+\mu \times 0=\left(p-m c_{f}\right) D(p)
\end{aligned}
$$

Hence, from (11), the negotiated affiliate fee is

$$
(1-\mu) \tau \times D(p)=(1-\zeta)\left(p-m c_{f}\right) D(p)-\zeta \mu \times\left(p-m c_{f}\right) D(p)
$$

so $(1-\mu) \tau=[(1-\zeta)-\zeta \mu]\left(p-m c_{f}\right)$. Thus, for a given $p$, an increase in $\mu$ lowers the effective affiliate fee $(1-\mu) \tau$ considered by $f$ when setting prices. The equilibrium price satisfies the fixed point condition that $p=\phi\left(m c_{f}+[(1-\zeta)-\zeta \mu]\left(p-m c_{f}\right)\right)$. Provided that the stability condition $\phi^{\prime}(\cdot)<1$ is satisfied, an increase in $\mu$ also lowers the equilibrium price $p$.

\footnotetext{
${ }^{38}$ Similar timing assumptions have been used in Nocke and White (2007), Draganska et al. (2010), and Ho and Lee (2017), and is also implicit in the analysis described in Rogerson (2014). In Appendix B, we also discuss how our external bargaining outcomes, given anticipated pricing and carriage decisions, can arise as the result of an alternating offer bargaining game of the form studied by Collard-Wexler et al. (2015).
} 


\section{Estimation and Identification}

In this section, we discuss the estimation of our model's parameters and how they are identified (given our modeling assumptions) from patterns in the data. We estimate all of our parameters jointly in a single step; however, for exposition, we discuss our estimation procedure in two steps:

1. We estimate $\boldsymbol{\theta} \equiv\left\{\boldsymbol{\theta}_{1}, \boldsymbol{\theta}_{2}, \boldsymbol{\theta}_{3}\right\}$, where:

(a) $\boldsymbol{\theta}_{1} \equiv\left\{\boldsymbol{\rho}, \boldsymbol{\nu}, \gamma^{d}, \gamma^{b}\right\}$, where $\boldsymbol{\rho} \equiv\left\{\rho_{c}^{0}, \rho_{c}^{1}\right\}_{\forall c}$ and $\boldsymbol{\nu} \equiv\left\{\nu^{S}, \nu^{N S}\right\}$, determines household viewership decisions by governing the distribution of $\gamma_{i c t}$, how fast marginal utilities from viewership decay, and the viewership utility reductions due to black-outs and distance to teams' stadiums;

(b) $\boldsymbol{\theta}_{2} \equiv\left\{\beta^{v}, \boldsymbol{\beta}^{x}, \boldsymbol{\rho}^{\text {sat }}, \alpha\right\}$, where $\boldsymbol{\rho}^{\text {sat }} \equiv\left\{\rho_{\text {DirecTV }}^{\text {sat }}, \rho_{\text {Dish }}^{\text {sat }}\right\}$, determines household distributor choice;

(c) $\boldsymbol{\theta}_{3} \equiv\left\{\mu, \zeta^{I}, \zeta^{E}, \sigma_{\omega}^{2}\right\}$ are parameters that affect firm incentives when pricing, bargaining, and determining carriage of channels. Recall that the parameter $\mu$ governs the extent to which integrated channels and distributors internalize profits across upstream and downstream units. Finally, $\sigma_{\omega}^{2}$ is the variance of an error term that influences MVPDs' carriage decisions in a manner that we discuss below.

2. We estimate (a lower bound for) our rival foreclosure parameter, $\lambda_{R}$.

To capture the impact of program access rules, we will assume that $\lambda_{R}=0$ in non-loophole markets and estimate our first step parameters using only these markets. That is, we assume that the program access rules effectively require integrated firms to ignore any foreclosure incentives in dealing with non-integrated rivals. ${ }^{39}$ We then estimate $\lambda_{R}$ using only the markets in our data in which RSNs took advantage of the terrestrial loophole (i.e., Philadelphia and San Diego).

Our estimation procedure conditions on the ownership structures of firms that are observed in the data. We maintain the assumption that the integration status of a channel or distributor does not directly affect viewership utility or distributor demand, and is not correlated with either measurement error (e.g., in affiliate fees or markups) or market-level profit disturbances considered by firms when bargaining or making pricing or carriage decisions. ${ }^{40}$

\footnotetext{
${ }^{39}$ We take this approach as a simple reduced-form way to capture the effects of program access rules. In practice, in markets subject to program access rules an integrated channel could attempt to deny access to a rival distributor at the risk of triggering a binding arbitration process in which the negotiated affiliate fees with other distributors might be used to determine the arbitrated price. Explicitly modeling this process is beyond the scope of the current analysis, and we leverage the assumption that $\lambda_{R}=0$ when PARs are enforced for tractability.

${ }^{40}$ This does not rule out the possibility that integrated channels may differ in quality from non-integrated channels (e.g., have different values of $\boldsymbol{\rho}_{c}$ ), as we estimate time varying channel taste parameters.
} 


\subsection{Estimation of Parameters $\boldsymbol{\theta}_{1}, \boldsymbol{\theta}_{2}, \boldsymbol{\theta}_{3}$}

\subsubsection{Moments used in Estimation}

We estimate the model parameters via GMM, using the following moments derived from the model described in the previous section.

Household Viewership. For every RSN and 38 national channels in each year, we use the difference between the following viewership moments observed in the data and predicted by the model: ${ }^{41}$

1. Summing across markets, the mean viewership for each channel-year;

2. Summing across markets, the number of households with zero viewership for each channelyear. $^{42}$

Household Distributor Choice. For every year and market, we assume that the unobservable characteristic for each distributor's bundle is orthogonal to a vector of instruments: i.e., $E\left[\xi_{f m t}(\boldsymbol{\theta}) \boldsymbol{Z}_{m t}\right]=0$, where the expectation is taken across all markets, firms, and years. For $\boldsymbol{Z}_{m t}$, we include: bundle observable characteristics $\boldsymbol{x}_{f m t}$; the maximum fraction of teams carried by the relevant RSNs in the market that are not blacked-out (to instrument for bundle utility $v_{f m t}^{*}$ ); and the satellite tax within the market, interacted with an indicator for whether the bundle is offered by a cable or satellite distributor (to instrument for bundle prices $p_{f m t}$ ). ${ }^{43}$ We recover $\xi_{f m t}(\boldsymbol{\theta})$ using the standard Berry et al. (1995) inversion.

Distributor Bargaining, Pricing, and Carriage. First, for any $\boldsymbol{\theta}$, the vector of affiliate fees $\left\{\tau_{f c t}\right\}$ and bundle-specific marginal costs $\left\{m c_{f m t}\right\}$ can be directly computed using the optimal pricing and bargaining conditions given by (5) and (10) (see Appendix C.2 for further details). We use these predicted values of $\left\{m c_{f m t}(\boldsymbol{\theta})\right\}$ and $\left\{\tau_{f c t}(\boldsymbol{\theta})\right\}$ in constructing the next set of moments which we form using only 2007 data and values:

1. Average affiliate fees: For each RSN active in 2007 and four national channels (ABC Family, ESPN, TNT, and USA), we minimize the difference between the model's predicted average affiliate fees across MVPDs and observed average affiliate fees: $E_{f}\left[\tau_{f c t}(\boldsymbol{\theta})\right]-\tau_{c t}^{o}$ (where variables with an $o$ superscript denote values of those objects that are observed in the data).

\footnotetext{
${ }^{41}$ To avoid re-solving the viewership problem for every household for every evaluation of a candidate parameter vector, we follow the importance sampling approach of Ackerberg (2009). See Appendix C.3 for further details.

${ }^{42}$ The MRI/Simmons data provides an estimate of the probability that a channel is never watched for national channels. We regress this probability on viewership, and use the estimated relationship to predict the probability that an RSN is never watched.

${ }^{43}$ The satellite tax changes that we use, by state year and percentage increase, are: CT 2003, 5\%; FL 2002, 10\%; KY 2006, 5\%; MA 2009, 5\%; NC 2003, 7\%; OH 2003, 6\%; and UT 2003, 5\%. We discuss these instruments further in Section 5.2 and in footnote 55.
} 
We weight estimated affiliate fees by national MVPD market shares conditional on observed carriage of the channel to approximate expectations across MVPDs.

Deviations in these and the next set of moments for implied markups reflect both measurement error in the data and sampling error, as our predictions are computed using a subset of U.S. markets.

2. Implied markups: For each distributor $f \in\{$ Comcast, DirecTV,Dish $\}$, we minimize the difference between the model's predicted MVPD price-cost margin and those observed in the data: $E_{m}\left[\left(p_{f m t}^{o}-m c_{f m t}(\boldsymbol{\theta})\right) / p_{f m t}^{o}\right]-\operatorname{markup} p_{f t}^{o}$.

3. RSN Carriage: Equation (6) implies that every cable distributor $f$ chooses the optimal set of channels (from among those with which it has agreements) to include in each market $m$ 's bundle. We assume that distributor $f$ 's true per household profits (not per subscriber) in market $m$ are given by $\tilde{\pi}_{f m t}^{M}(\cdot)$, where:

$$
\tilde{\pi}_{f m t}^{M}\left(\mathcal{B}_{m t}, \cdot\right) \equiv\left[\pi_{f m t}^{M}\left(\mathcal{B}_{m t}, \cdot\right)-\omega_{f m t}\left(\mathcal{B}_{f m t}\right)\right]
$$

and $\pi_{f m t}^{M}\left(\mathcal{B}_{m t}, \cdot\right)$ represents our (the econometrician's) estimate of a firm's per household profits. The term $\omega_{f m t}\left(\mathcal{B}_{f m t}\right)$ represents a mean-zero i.i.d. bundle-distributor-market-time specific disturbance; we assume that $\omega_{f m t}(\cdot) \sim N\left(0, \sigma_{\omega}^{2}\right) .{ }^{44}$

If channel $c$ has negotiated an agreement with some firm $f$ : (i.e., $f$ carries $c$ on its bundles in some non-empty set of markets), then firm $f$ 's optimal carriage decision given by (6) implies that:

$$
\begin{array}{ll}
\left(\left[\Delta_{f c} \pi_{f m t}^{M}\left(\mathcal{B}_{m t} \cup f c, \cdot\right)\right]-\left[\Delta_{f c} \omega_{f m t}\left(\mathcal{B}_{f m t} \cup f c, \cdot\right)\right]\right) \geq 0 & \forall m: c \in \mathcal{B}_{f m t}, \\
\left(\left[\Delta_{f c} \pi_{f m t}^{M}\left(\mathcal{B}_{m t} \cup f c, \cdot\right)\right]-\left[\Delta_{f c} \omega_{f m t}\left(\mathcal{B}_{f m t} \cup f c, \cdot\right)\right]\right) \leq 0 & \forall m: c \notin \mathcal{B}_{f m t},
\end{array}
$$

where $\left[\Delta_{f c} \pi_{f m t}^{M}\left(\mathcal{B}_{m t}, \cdot\right)\right] \equiv \pi_{f m t}^{M}\left(\mathcal{B}_{m t}, \cdot\right)-\pi_{f m t}^{M}\left(\mathcal{B}_{m t} \backslash f c, \cdot\right),\left[\Delta_{f c} \omega_{f m t}\left(\mathcal{B}_{f m t}\right)\right] \equiv \omega_{f m t}\left(\mathcal{B}_{f m t}\right)-$ $\omega_{f m t}\left(\mathcal{B}_{f m t} \backslash f c\right)$, and $\mathcal{B}_{m t} \cup f c$ denotes the set of all bundles $\mathcal{B}_{m t}$ where $c$ is added to bundle $f .{ }^{45}$ That is, these inequalities imply that in any market in which $c$ is carried by $f, f$ obtains higher profits from carrying than by dropping $c$ (holding fixed prices and carriage decisions of other firms); similarly, in any market where $c$ is not carried, $f$ obtains higher profits from not carrying than by carrying $c$.

Given our assumptions on the distribution of $\omega_{f m t}(\cdot)$, it follows that:

$$
\operatorname{Pr}\left(c \in \mathcal{B}_{f m t}\right)=\Phi\left(\left[\Delta_{f c} \pi_{f m t}^{M}\left(\mathcal{B}_{m t} \cup f c, \cdot\right)\right] /\left(2 \sigma_{\omega}\right)\right),
$$

\footnotetext{
${ }^{44}$ We interpret $\omega_{f m t}(\cdot)$ as the difference between our estimated profits and those used by a local system operator when determining carriage decisions; we assume that these disturbances are not accounted for by a distributor when pricing or bargaining with channels.

${ }^{45}$ In cases where $c \in \mathcal{B}_{\text {fmt }}$, this definition implies that $\mathcal{B}_{m t} \cup f c=\mathcal{B}_{m t}$.
} 
Table 1: Regression of RSN Carriage on Integration Status, Distance, and Blackout Percentage

\begin{tabular}{lcccc}
\hline & $(1)$ & $(2)$ & $(3)$ & $(4)$ \\
\hline VI Ownership Share & $0.404^{* * *}$ & $0.435^{* * *}$ & $0.293^{* * *}$ & $0.171^{* *}$ \\
& $(0.0674)$ & $(0.0837)$ & $(0.110)$ & $(0.0852)$ \\
\% Teams not Blacked Out & $0.412^{* * *}$ & $0.399^{* * *}$ & $0.429^{* * *}$ & $0.477^{* * *}$ \\
& $(0.0494)$ & $(0.0586)$ & $(0.109)$ & $(0.107)$ \\
Avg Distance to RSN's Stadiums & $-0.559^{* * *}$ & $-0.630^{* * *}$ & $-0.838^{* * *}$ & $-0.795^{* * *}$ \\
$\left(10^{3}\right.$ mi & $(0.100)$ & $(0.117)$ & $(0.238)$ & $(0.284)$ \\
& & & & \\
Years & $2000-10$ & 2007 & 2007 & 2007 \\
Systems & All Systems & All Systems & Has P Q & Has P Q \\
Has Deal & No & No & No & Yes \\
Observations & 154,121 & 12,246 & 1,132 & 1,052 \\
R-squared & 0.615 & 0.616 & 0.670 & 0.639 \\
\hline
\end{tabular}

Notes: Linear probability regression where the dependent variable is whether a cable system carries an RSN in a relevant market in 2007. Specifications differ by sample used, where "Has P Q" restricts attention to systems for which price and quantity data is available, and "Has Deal" restricts attention to system-RSN pairs where the MVPD has a deal with the RSN (i.e., carries the RSN on at least one other system). All specifications use DMA, RSN and (when appropriate) year fixed effects. Inclusion of system demographic controls (race, population density, average income, household ownership) did not appreciably change point estimates. ${ }^{* * *} \mathrm{p}<0.01,{ }^{* *} \mathrm{p}<0.05,{ }^{*} \mathrm{p}<0.1$. Standards errors are reported in parenthesis, and are clustered by DMA.

where $\Phi$ is the standard normal cumulative distribution function.

We construct several moments based on the model's predicted carriage probabilities. First, we construct moments based on indirect inference (cf. Gouriéroux and Monfort (1996)) that match the predicted to observed relationship between carriage of a relevant RSN by a system and (i) the ownership share of the RSN by the system's MVPD, (ii) the distance of the system to the RSN's teams' stadiums, and (iii) the fraction of teams on the RSN that are not blacked-out. Table 1 presents the results of a linear probability regression predicting whether a cable system carries a relevant RSN in our data. We find that carriage of an RSN by a cable system is increasing with the share of the RSN owned by the system's MVPD, and decreasing in the distance between the system and the RSN's teams' stadiums and in the fraction of teams that are blacked-out. We perform the same regression using the predicted carriage probabilities from our model, and match the estimated coefficients for vertical integration, distance, and the fraction of teams not blacked-out from this regression to the coefficients in specification (4) in Table $1 .^{46}$

Second, we calculate the probability that an RSN is carried by a cable distributor in a relevant market, and match the probability that is observed in the data to that predicted by our model via (15). ${ }^{47}$ Third, we set $\partial \mathcal{L}_{\text {carriage }} / \partial \sigma_{\omega}=0$, where $\mathcal{L}_{\text {carriage }}$ is the predicted log-likelihood

\footnotetext{
${ }^{46}$ We focus on the "Has Deal" specification as our model does not predict the probability of carriage for a system if the MVPD and channel do not have a deal.

${ }^{47}$ E.g., if there are only two RSNs $A$ and $B$, and $A$ is carried on cable in $30 / 40$ of $A$ 's relevant markets, and $B$ is carried on cable in $25 / 60$ of $B$ 's relevant markets, the probability than an RSN is carried by a cable distributor in a relevant market is 0.55 .
} 
of the observed market-level RSN carriage decisions by cable MVPDs, given by:

$$
\mathcal{L}_{\text {carriage }}=\sum_{c \in \mathcal{C}_{t}^{R}} \sum_{f m: c \in \mathcal{A}_{f t}}\left(1_{\left\{c \in \mathcal{B}_{\text {fmt }}\right\}} \times \log \operatorname{Pr}\left(c \in \mathcal{B}_{f m t}\right)+1_{\left\{c \notin \mathcal{B}_{f m t}\right\}} \times \log \operatorname{Pr}\left(c \notin \mathcal{B}_{f m t}\right)\right)
$$

where $\mathcal{C}_{t}^{R}$ denotes the set of RSNs, and $\mathcal{A}_{f t}$ are the set of channels available to MVPD $f$.

\subsubsection{Identification of Parameters $\theta_{1}, \theta_{2}$ and $\theta_{3}$}

We now provide a discussion of how these moments and variation in the data help identify the parameters of our model (given our assumptions and functional forms that are employed).

Viewership and Distributor Choice Parameters $\left(\boldsymbol{\theta}_{1}, \boldsymbol{\theta}_{2}\right)$. The main parameters governing the distribution of channel taste parameters $\gamma_{i c t}$ (i.e., $\boldsymbol{\rho}$ ) are primarily identified from viewing behavior: channels that are watched at all by a larger fraction of households have higher values of

$\rho_{c}^{0}$ (the probability that $\gamma_{i c t}>0$ ), and those that conditional upon being watched are watched more often have higher values of $\rho_{c}^{1}$ (the mean of the distribution). However, since we do not possess ratings for RSNs at the market level, we identify the black-out and distance parameters $\left(\gamma^{b}\right.$ and $\gamma^{d}$ ) from the RSN Carriage moments; we defer discussion of these parameters until the end of this subsection when discussing identification of $\mu$.

Parameters governing household bundle choice $\left(\boldsymbol{\beta}^{x}\right.$ and $\left.\boldsymbol{\beta}^{v}\right)$ are identified from variation in bundle market shares as observed bundle characteristics (including its viewership utility) changes; Table A.1 summarizes the substantial variation in prices and channel carriage across markets. Viewership utility varies as a function of the set of channels that are carried and, if an RSN is carried, whether the market is further from teams' stadiums or if teams carried are blacked-out. Since firms may respond to local demand shocks when determining carriage, we instrument for viewership utility using the fraction of blacked-out teams on RSNs in each relevant market. The satellite tax is an instrument for price, and is used to identify the price sensitivity coefficient $\alpha$. Information contained in cable and satellite pricing margins helps identify the heterogeneity in preferences for satellite. In particular, the relationship between satellite and cable market shares has strict implications for predicted price elasticities (and hence implied markups) under a standard logit demand system without preference heterogeneity; inclusion of a random preference for satellite (parameterized by $\rho^{\text {sat }}$ ) assists with rationalizing observed markups for a given satellite market share.

In addition to observing how bundle market shares vary based on channel composition (which has limited variation for some channels across markets), matching observed average affiliate fees negotiated for each channel $\left\{\tau_{c t}^{o}\right\}$ to those predicted by the model $\left\{\tau_{f c t}(\boldsymbol{\theta})\right\}$ is crucial for identifying the values that consumers place on channels. First, our model relates $\tau_{f c t}(\boldsymbol{\theta})$ to the gains from trade created when channel $c$ contracts with firm $f$ : i.e., differences in $f$ and $c$ 's profits (primarily realized from subscription and advertising revenues) when $f$ drops $c$. Thus, our model attempts to rationalize a channel with higher observed affiliate fees $\tau_{c t}^{o}$ by predicting that this channel creates 
greater demand through the term $\beta^{v} v_{i f t}^{*}$ in a household's distributor utility equation given by (2), which in turn is also a function of parameters governing the distribution of $\gamma_{i c t}$, and how $\gamma_{i c t}$ is scaled to enter into utility by a "decay parameter" (either $\nu_{c}^{S}$ for sports channels or $\nu_{c}^{N S}$ for nonsports channels) - i.e., a channel with a higher $\gamma_{i c t}$ and lower decay parameter than another will contribute more to a viewer's utility from the same amount of time the channel is watched. ${ }^{48}$

Our choice to allow different values of decay parameters for sports and non-sports channels is motivated by the data, illustrated in Figure 3. Sports channels have consistently higher negotiated affiliate fees than non-sports channels with similar viewership patterns (ratings), often receiving payments an order of a magnitude higher. Our model rationalizes this fact by assigning a higher decay rate to sports channels than to non-sports channels. This implies that, for a given amount of time spent watching, consumers derive higher utility from sports than non-sports channels, and sports channels obtain higher affiliate fees due to the greater gains from trade. Furthermore, levels of decay parameters are identified by the change in viewership of channels as the availability of other channels varies, and by the variation in ratings and fees within sports or non-sports channels: e.g., a higher value of $\nu^{S}$ reduces the amount by which greater viewership for a sports channel translates into higher generated utility and, hence, higher affiliate fees.

To anchor the discussion in the past two paragraphs in an example, consider a single market and bundle with two non-integrated channels $c$ and $d$, and a single household $i$; ignore the time index $t$. Assume that the household watches $d$ more than $c$. This could be induced by many potential combinations of $\left(\gamma_{i c}, \nu_{c}, \gamma_{i d}, \nu_{d}\right)$. For example, $\gamma_{i d}$ could be higher than $\gamma_{i c}$ and $\nu_{c}=\nu_{d}$. If this were true, however, then $d$ should obtain higher negotiated affiliate fees as it would be predicted to generate a higher surplus for a viewer, and hence there would be greater gains from trade from carriage of $d$ than $c$. However, if affiliate fees are observed to be the same for the two channels despite the difference in viewership, then the model would predict that the rate of "decay" $\left(\nu_{c}\right)$ and initial utility $\left(\gamma_{c}\right)$ for channel $c$ were in fact higher than for channel $d$, thereby allowing $c$ to generate the same utility for consumers - and hence the same negotiated affiliate fees - for the shorter amount of time watched.

Bargaining, Pricing, and Carriage Parameters $\left(\boldsymbol{\theta}_{3}\right)$. Although the internalization parameter $\mu$ enters into the computation of several moments (including any moment based off of recovered values of $\tau_{f c t}(\boldsymbol{\theta})$ and $\left.m c_{f m t}(\boldsymbol{\theta})\right)$, we expect it is identified primarily off of the RSN Carriage moments. As $\mu$ increases, distributors have a greater incentive to carry a channel for a fixed value of $\tau_{f c t}(\cdot)$, (positive) level of integration $\left(O_{f c t}>0\right.$ ), and the contribution of the channel to downstream profits. Hence, this parameter will help to rationalize higher carriage rates between integrated distributors and channels observed in the data and captured in the regression coefficients reported in Table 1. Black-out and distance parameters, $\gamma^{b}, \gamma^{d}$, are identified in a similar fashion.

An example of the variation in the data that we leverage is illustrated in Figure 4, which presents

\footnotetext{
${ }^{48}$ For computational reasons, during estimation we restrict $\nu^{S}$ to lie on a coarse grid while allowing all other parameters to vary freely; see Appendix C.3 for further details and robustness tests. See also the discussion in the appendix of Crawford and Yurukoglu (2012) which examines a variant of this model using Monte Carlo simulation.
} 


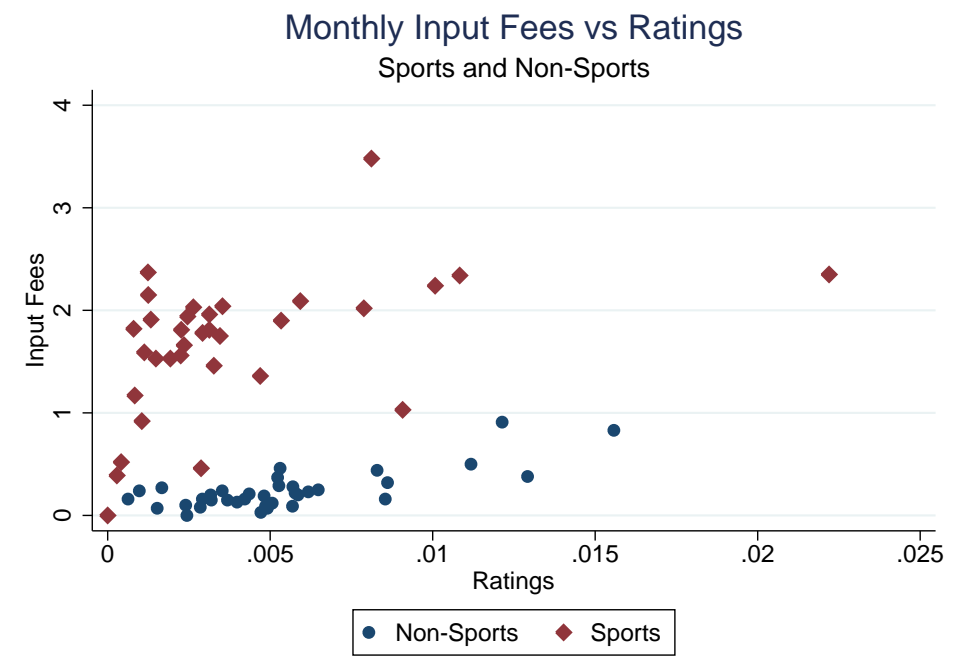

Figure 3: Negotiated monthly affiliate fees and viewership ratings.

the integrated and non-integrated carriage of a Comcast integrated RSN in three different regions of the U.S. In these three settings, cable systems in markets close to the RSN's teams' stadiums almost always carry the RSN; systems far away most often do not. However, in markets located a moderate distance away from these stadiums, these RSNs are much more likely to be carried on systems owned by Comcast than on non-integrated systems. For example, in Figure 4a, all Comcast systems in northern Vermont carry CSN New England (denoted by black dots) whereas most non-Comcast systems (denoted by grey dots for systems that carry the RSN, and grey X's for those that do not) do not carry CSN New England; and in Figures 4b and 4c, non-carriage by non-Comcast systems occurs much closer to the RSN's teams' stadiums than for Comcast systems (as there is a higher ratio of grey X's to grey dots near Washington DC and Chicago than of black X's, denoting non-carriage by Comcast systems, to black dots). These maps also indicate that non-carriage is much more likely in areas where the teams on the RSN are blacked-out (as in New York for CSN New England, Pennsylvania for CSN Mid-Atlantic, and Michigan for CSN Chicago).

Next, different values for the Nash bargaining parameters $\left\{\zeta^{I}, \zeta^{E}\right\}$ affect the model's ability to match the relationship between factors influencing MVPD and channel "gains from trade" with negotiated input fees. For example, consider a non-integrated MVPD $f$ and $\operatorname{RSN} c$. If $\zeta^{E}=0$ (so that the Nash bargaining parameter for distributors is equal to 0 when bargaining with a nonintegrated channel), the bargaining first-order conditions given by (11) imply that input fees $\tau_{f c t}$ would be determined solely by MVPD f's gains from trade from carrying channel $c\left(G F T_{f c t}^{M}\right)$, and not by channel $c$ 's gains from trade $\left(G F T_{f c t}^{C}\right)$. This implies that channel $c$ 's advertising revenues $a_{c t}$, which enter only into $G F T_{f c t}^{C}$, would not affect negotiated input fees if $\zeta^{E}=0$. Thus, controlling for viewership, the extent to which observed channel affiliate fees vary with advertising revenues identifies $\zeta^{E}$.

Finally, we identify the variance of carriage disturbances $\sigma_{\omega}^{2}$ from our carriage moments. Recall 


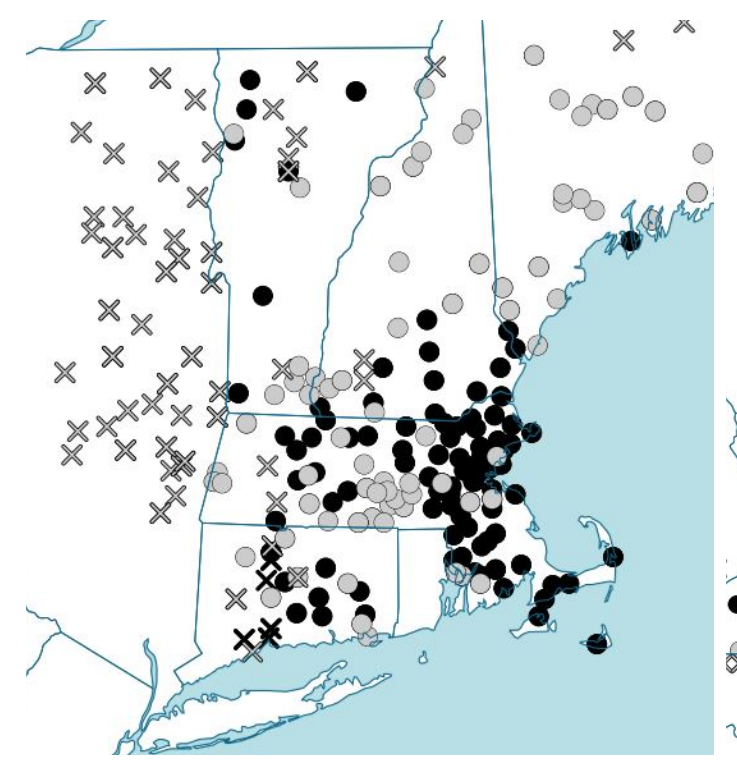

(a) Carriage of CSN New England

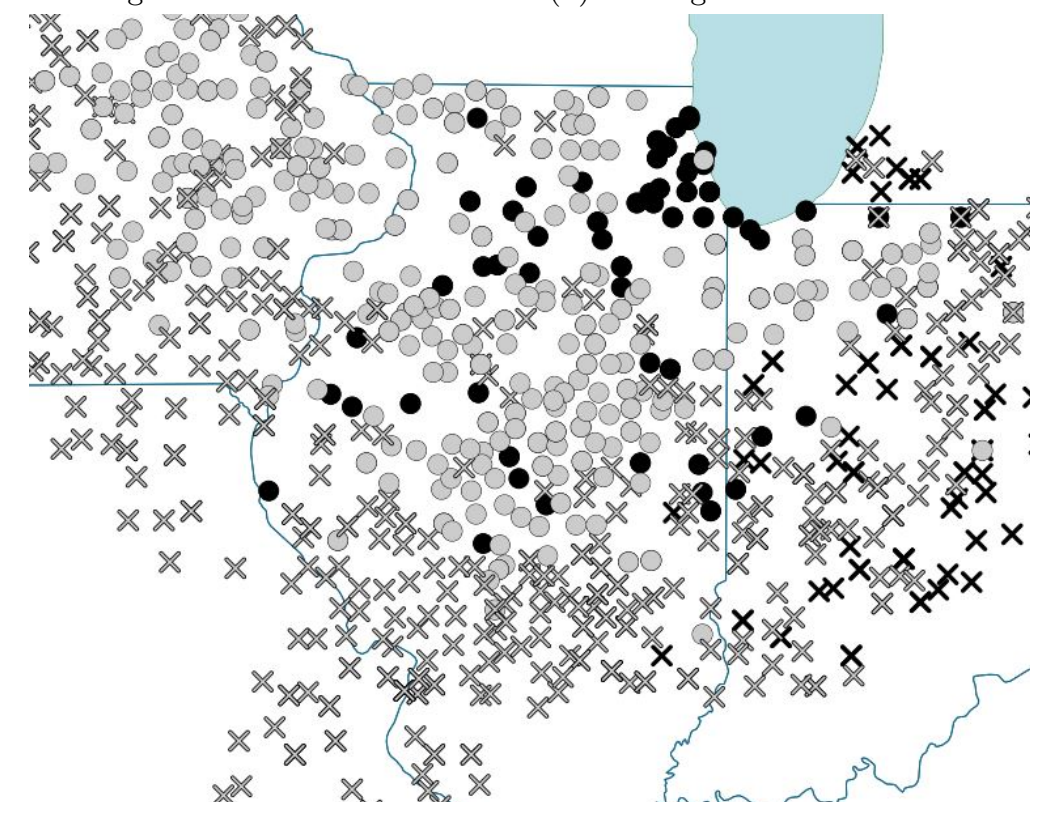

(c) Carriage of CSN Chicago

Figure 4: Carriage by Comcast and non-integrated cable MVPDs of three Comcast-integrated RSNs across cable systems in 2007. Dots represent carriage by a system, X's represent no carriage. Black markers represent Comcast systems, grey markers represent non-Comcast cable systems.

that these carriage moments include matching the overall probability that an RSN is carried by a cable distributor in a relevant market, and setting the derivative of the log-likelihood of observing the carriage decisions in the data with respect to $\sigma_{\omega}$ to zero. As this variance increases while holding all other parameters fixed, the predicted carriage probability for any channel approaches $1 / 2$ as carriage decisions become based purely on noise. Thus, lower values of $\sigma_{\omega}^{2}$ indicate that our model's predicted changes in distributor's profits from carrying a channel can be used to predict 
observed carriage decisions. Note also that while $\sigma_{\omega}^{2}$ can be estimated using information on carriage rates for non-integrated firms only, the identification argument for our internalization parameter $\mu$ relies on carriage rates for both non-integrated and integrated firms.

\subsection{Estimation of Rival Foreclosure Parameter $\lambda_{R}$}

To recover lower bounds for our rival foreclosure parameter $\lambda_{R}$, we will use information provided by markets in which distributors are able to exclude competitors from carrying an integrated RSN channel-i.e., terrestrial loophole markets. The markets we focus on are Philadelphia and San Diego, the channels in question CSN Philadelphia (owned by Comcast) and 4SD (owned by Cox), and the competitors excluded from carriage are satellite distributors DirecTV and Dish.

Observe that because these two markets both had total exclusion of satellite distributors, we will only be able to estimate a lower bound on $\lambda_{R}$, which we will denote by $\underline{\lambda}_{R}$. Intuitively, this lower bound will be the lowest level of $\lambda_{R}$ at which there are no mutual gains from trade between the RSN and either satellite distributor (i.e., at which the gains from exclusion exceed the gains from carriage). In general, however, whether there are gains from trade between an RSN and a satellite distributor depends on the satellite firm's beliefs about whether, if it is supplied, the other satellite firm will also be supplied. In Appendix B we show that a necessary condition for non-supply, regardless of the satellite firm's beliefs, is that the joint profit of the RSN $c$ and the two satellite firms $g$ and $g^{\prime}$ is reduced when both satellite firms have access to the RSN, which can be stated as:

$$
\begin{gathered}
\sum_{m \in \mathcal{M}_{c}}\left[\left[\Delta_{g c, g^{\prime} c} \Pi_{g m t}^{M}\left(\left\{\mathcal{B}_{m t}^{o} \cup\left\{g c, g^{\prime} c\right\}\right\}, \boldsymbol{p}_{m t}^{o}, \tilde{\boldsymbol{\tau}} ; \hat{\mu}\right)\right]+\left[\Delta_{g c, g^{\prime} c} \Pi_{g^{\prime} m t}^{M}\left(\left\{\mathcal{B}_{m t}^{o} \cup\left\{g c, g^{\prime} c\right\}\right\}, \boldsymbol{p}_{m t}^{o}, \tilde{\boldsymbol{\tau}} ; \hat{\mu}\right)\right] \ldots\right. \\
\left.+\left[\Delta_{g c, g^{\prime} c} \Pi_{c m t}^{C}\left(\left\{\mathcal{B}_{m t}^{o} \cup\left\{g c, g^{\prime} c\right\}\right\}, \boldsymbol{p}_{m t}^{o}, \tilde{\boldsymbol{\tau}} ; \hat{\mu}, \lambda_{R}\right)\right]\right] \leq 0
\end{gathered}
$$

where the left-hand side of the inequality, which we refer to as the "three-party surplus," represents $g, g^{\prime}$, and $c$ 's joint gains from trade from both $g$ and $g^{\prime}$ being supplied with channel $c$ and carrying the channel in all of $g$ 's relevant markets, and $\tilde{\boldsymbol{\tau}}$ equals the predicted values of affiliate fees $\hat{\boldsymbol{\tau}}(\cdot)$ except that $\tilde{\tau}_{g c t}=\tilde{\tau}_{g^{\prime} c t}=0 .{ }^{49}$

We can therefore estimate a lower bound for $\lambda_{R}$ that holds for any beliefs held by a satellite firm about whether the RSN will also be supplied to the rival satellite firm by finding the lowest value $\underline{\lambda}_{R}$ that makes (16) hold for both of the loophole-market cable-integrated RSNs that do not contract with the satellite distributors (CSN Philadelphia and 4SD). ${ }^{50}$ We estimate a separate lower

\footnotetext{
${ }^{49}$ Specifically, we show that in an alternating offer bargaining game of the form studied by Collard-Wexler et al. (2015), if the three-party-surplus is positive, then RSN $c$ has a deviating pair of offers $\left\{\tilde{\tau}_{g c}, \tilde{\tau}_{g^{\prime} c}\right\}$ to both satellite distributors that both will accept regardless of their beliefs over whether, if they are supplied, their rival will also be supplied, and will increase $c$ 's profits. See Appendix B for a formal derivation and discussion of the idea behind this result.

${ }^{50} \mathrm{An}$ alternative would be to assume that when approached by the RSN to negotiate supply, a satellite firm holds
} 
bound for each market (i.e., $\underline{\lambda}_{R}^{P h i l}$ and $\underline{\lambda}_{R}^{S D}$ ).

Incentives for Exclusion. It is instructive at this point to discuss the competing forces that would induce a cable provider to withhold its integrated RSN from a satellite provider. This is equivalent to understanding why the gains created when satellite distributors are supplied with the RSN may be offset by the losses incurred by the integrated cable provider.

The primary gains created when a satellite distributor $g$ is supplied with the RSN are through potential market expansion effects from carriage: i.e., if consumers who previously did not subscribe to an MVPD now would if satellite were to carry the RSN. Each household that substitutes from the outside good to $g$ would generate additional industry profit equal to the level of $g$ 's margins plus any additional advertising revenues generated by those households watching the RSN.

The primary losses generated by supplying $g$ with the RSN would be incurred by the RSN's integrated cable distributor if households substituted away from the integrated cable provider to $g$. Although these consumers would generate profit for $g$, insofar as cable margins are higher than those of satellite providers (by 10+ percentage points in our data), any household that switched from cable to satellite as a result of supplying satellite with the RSN would reduce industry profit by this difference in margins.

Consequently, factors that make exclusion of satellite by an integrated cable owner (for $\lambda_{R}>0$ ) more likely include: (i) a smaller share of consumers that are not subscribers to any MVPD and lower advertising rates (thereby reducing the potential gains generated by market expansion); (ii) a larger cable "footprint" (market share) in the RSN's relevant market area; (iii) closer substitutability between satellite and cable distribution; and (iv) a larger differential between cable and satellite margins (all of which would exacerbate the losses from business stealing by satellite from cable). For all such factors, lower values of $\lambda_{R}$ (closer to 0 ) cause any losses incurred by the RSN's integrated owner to be internalized less by the RSN when bargaining with $g$, reducing the likelihood of exclusion occurring.

\section{$5 \quad$ Results and Parameter Estimates}

Estimates of selected key parameters of our model are reported in Table 2. We discuss our estimates primarily through how they influence predicted moments relating to consumer viewership and subscription patterns, firm pricing and carriage decisions, and negotiated agreements.

\subsection{Viewership Parameters}

The willingness-to-pay (WTP) for each channel can be derived by computing the contribution of a given channel to bundle utility for each household $\left(v_{i j t}^{*}\right.$ in $(2)$ ), multiplying it by our estimates of parameters $\beta^{v} / \alpha$ to convert it into dollars, and averaging across households (as households have

the belief that the rival satellite firm will not be supplied. The approach we employ instead provides a lower bound for $\lambda_{R}$ that holds for any beliefs. 
Table 2: Estimates of Key Parameters

\begin{tabular}{|c|c|c|c|c|}
\hline & Parameter & Description & Estimate & SE \\
\hline Viewership & $\nu^{N S}$ & Viewership Decay, Non-sports & 0.59 & 0.00 \\
\hline Parameters & $\nu^{S}$ & Viewership Decay, Sports & 0.95 & - \\
\hline \multirow[t]{2}{*}{$\boldsymbol{\theta}_{1}$} & $\gamma^{b}$ & Fraction of Teams Blacked-out & -0.58 & 0.31 \\
\hline & $\gamma^{d}\left(10^{3} \mathrm{mi}\right)$ & Distance & -0.93 & 0.27 \\
\hline Bundle Choice & $\alpha$ & Bundle Price & -1.00 & 0.44 \\
\hline Parameters & $\beta^{v}$ & Bundle Viewership Utility & 0.14 & 0.07 \\
\hline \multirow{2}{*}{$\boldsymbol{\theta}_{2}$} & $\rho_{\text {DirecTV }}^{\text {sat }}\left(10^{2}\right)$ & DirecTV Exponential Parameter & 0.42 & 0.23 \\
\hline & $\rho_{\text {Dish }}^{\text {sat }}\left(10^{2}\right)$ & Dish Exponential Parameter & 0.49 & 0.27 \\
\hline Pricing, Bargaining, & $\sigma_{\omega}^{2}$ & Variance of Carriage Shocks & 0.00 & 0.00 \\
\hline Carriage and & $\zeta^{E}$ & Bargaining, External & 0.28 & 0.03 \\
\hline Foreclosure Parameters & $\zeta^{I}$ & Bargaining, Internal & 0.37 & 0.06 \\
\hline \multirow[t]{3}{*}{$\boldsymbol{\theta}_{3}, \underline{\lambda}_{R}$} & $\mu$ & Internalization & 0.79 & 0.09 \\
\hline & $\mu \times \underline{\lambda}_{R}^{P h i l}$ & Internalization \& Rival Foreclosure, Philadelphia & 1.11 & 0.14 \\
\hline & $\mu \times \underline{\lambda}_{R}^{S D}$ & Internalization \& Rival Foreclosure, San Diego & 0.94 & 0.11 \\
\hline
\end{tabular}

Notes: Selected key parameters from the first and second step estimation of the full model, where parameter $\nu^{S}$ is estimated separately via a grid search (see Appendix C.3). Additional viewership parameters contained in $\boldsymbol{\theta}_{1}$ are reported in Appendix Table A.4; state-firm and year fixed effects in $\boldsymbol{\theta}_{2}$ are not reported. Asymptotic GMM standard errors are computed using numerical derivatives and 1500 bootstrap draws of markets and simulated households to estimate the variance-covariance matrix of the moments.

different tastes $\left(\gamma_{i c t}\right)$ for each channel, which are distributed according to parameters $\left.\boldsymbol{\rho}\right) .{ }^{51}$ We report estimated values of these parameters and WTPs in 2007 for all channels in Appendix Table A.4. We also depict the distribution of household WTPs for nine national channels, conditional on being positive, in Figure 5a, with the fraction of households with positive valuations listed for each channel. Although most national channels have average WTP values below $\$ 1$ per month (and other than sports channel ESPN, none exceed $\$ 2$ ), the pattern is very different for RSNs: none are predicted to have average WTP values less than $\$ 1$ per month, and over $70 \%$ are greater than $\$ 2$.

Our estimates of the RSN distance-decay parameter $\gamma^{d}$ and blackout parameter $\gamma^{b}$ are negative, and imply that consumers derive less utility from watching an RSN both (i) the further they are from the teams carried on the RSN, and (ii) the greater the fraction of teams that are blacked out. We predict that increasing the average distance of a household from an RSN's teams' stadiums from 0 to 100 miles reduces that household's value of the channel by approximately $9 \% .^{52}$ Figure $5 \mathrm{~b}$ illustrates this pattern, and plots the predicted average WTP in 2007 for four different RSNs as the distance from a household to an RSN's teams' stadiums increases. ${ }^{53}$ Similarly, we predict that subjecting half of the teams that an RSN normally broadcasts to blackout restrictions reduces consumers' valuation of the channel by $25 \%$.

Finally, we estimate $\nu^{N S}$ to be different than $\nu^{S}$. The lower estimated value of $\nu^{N S}$ implies

\footnotetext{
${ }^{51}$ We compute the average WTP for channels relative to a synthetic bundle that includes every national channel carried by at least $60 \%$ of systems in 2007 , and by using 20,000 simulated households. When computing the WTP for an RSN $c$, we add the RSN to the synthetic bundle and use the average values of $b_{i c t}$ and $d_{i c}$ across all markets that carry the RSN.

${ }^{52} \mathrm{As}$ distance is measured in thousands of miles, being further away by 100 miles scales utility by $\exp \left(\hat{\gamma}^{d} \times 0.1\right)$.

${ }^{53}$ Each point in Figure 5b corresponds to a market in which the RSN is carried in 2007, and the WTP for each market is computed by averaging over 160 simulated households per market using that market's value of $b_{i c t}$ and $d_{i c}$.
} 

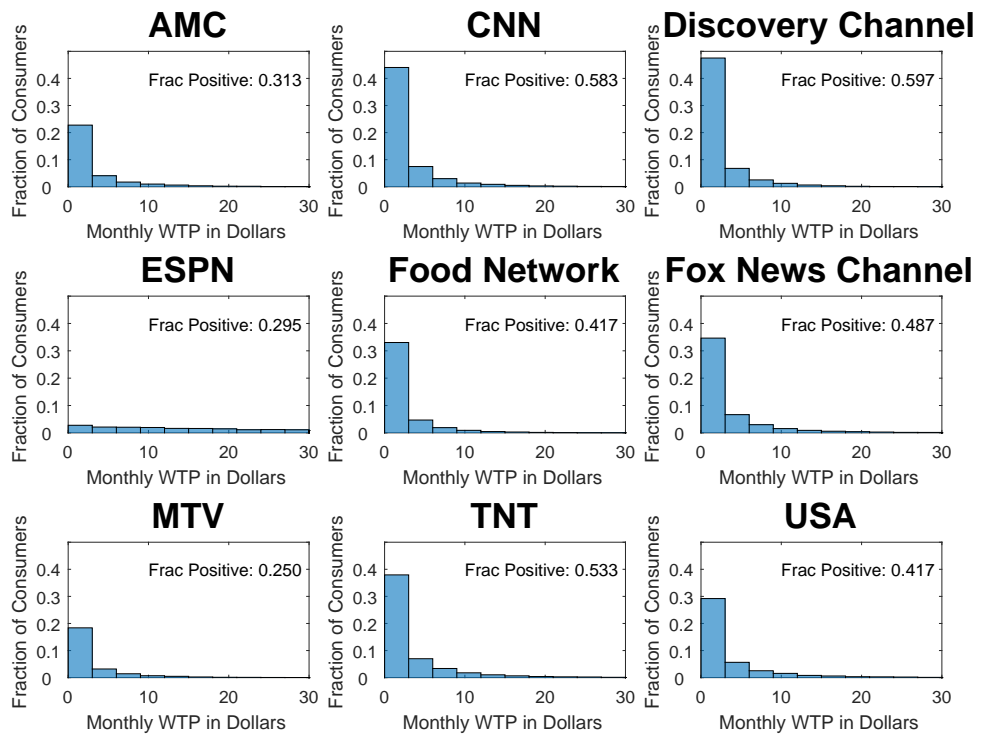

(a) Histograms of monthly WTP (conditional on being positive) for selected national channels.
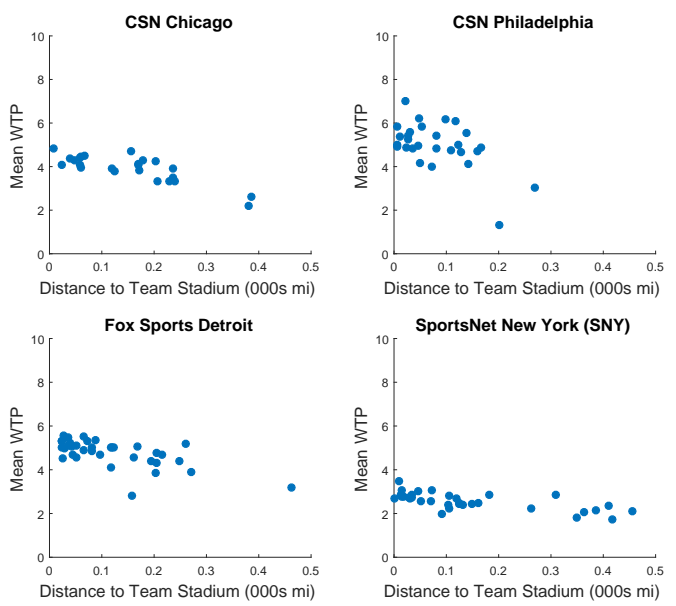

(b) Mean WTP in a market versus distance to RSN's teams' stadiums, for four RSNs.

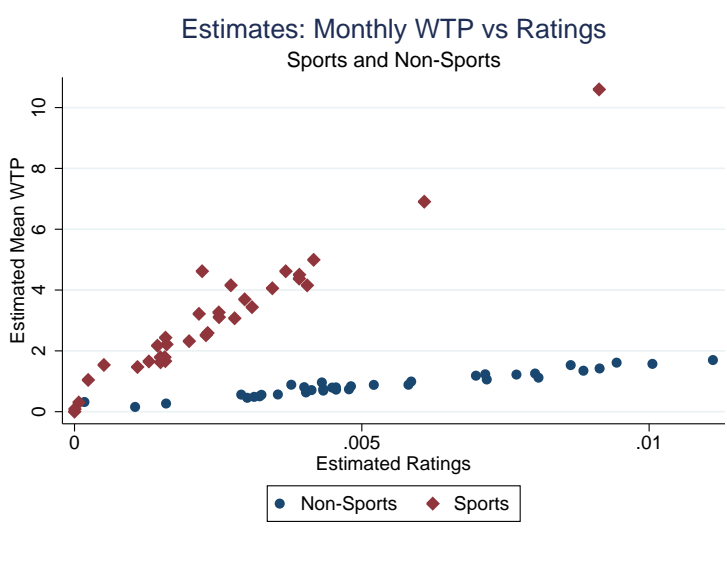

(c) Estimated monthly WTP versus estimated ratings for sports and non-sports channels

Figure 5: Predicted willingness-to-pay (WTP) for channels (2007 values).

that consumers' marginal utility from watching non-sports channels falls slower than for sports channels; in turn, this implies that consumers derive higher utility from sports channels than nonsports channels if they choose to spend the same amount of time spent watching each. Our model thus predicts that sports channels receive higher negotiated affiliate fees for the same viewership ratings, as depicted in Figure 5c for the year 2007. 
Table 3: Elasticities and Margins

\begin{tabular}{|c|c|c|c|}
\hline Elasticity of row with respect to price of column: & Cable & DirecTV & Dish \\
\hline Cable & -1.69 & 0.30 & 0.19 \\
\hline DirecTV & 2.16 & -2.90 & 0.13 \\
\hline Dish & 3.18 & 0.22 & -4.15 \\
\hline Outside Option & 5.52 & 0.26 & 0.16 \\
\hline \multicolumn{4}{|l|}{ Predicted Margins } \\
\hline Mean Comcast Margin & 0.66 & & \\
\hline Mean DirecTV Margin & 0.48 & & \\
\hline Mean Dish Margin & 0.45 & & \\
\hline \multicolumn{4}{|l|}{ Logit Demand Price Coefficients } \\
\hline OLS Logit Price Coefficient & $-0.004^{* *}$ & $(0.002)$ & \\
\hline IV Logit Price Coefficient & $-0.080 * * *$ & $(0.025)$ & \\
\hline $\begin{array}{l}\text { Semi-Elasticity of row with respect to } \\
\text { removal of ESPN from column: }\end{array}$ & Cable & DirecTV & Dish \\
\hline Cable & -18.90 & 3.86 & 2.36 \\
\hline DirecTV & 54.82 & -19.52 & 1.11 \\
\hline Dish & 51.16 & 1.85 & -19.67 \\
\hline Outside Option & 17.27 & 0.22 & 0.14 \\
\hline
\end{tabular}

Notes: This table reports predicted mean price elasticities, predicted margins for Comcast and the two satellite distributors, the estimated price coefficient from a logit demand regression without (OLS) and with (IV) the use of price instruments (where standard errors clustered at market level), and semi-elasticities from dropping the national channel ESPN. For logit demand estimates, $* *, * * *$ represent significance at the $5 \%$ and $1 \%$ levels.

\subsection{Distributor Choice Parameters}

All reported coefficients in $\boldsymbol{\theta}_{2}$ are statistically significant at the $5 \%$ level, and have the expected sign: consumers negatively respond to price $(\alpha)$, and positively respond to the indirect utility they receive from a bundle's channels $\left(\beta^{v}\right)$.

At the top of Table 3 we report the average own- and cross-price elasticities that are predicted by our model. Demand for the average cable system is more inelastic (-1.7) than for satellite $(-2.9$ and -4.2), which is consistent with higher cable market shares and margins that are both observed in the data and predicted by our model. ${ }^{54}$ Estimated values of $\rho_{\text {DirecTV }}^{\text {sat }}$ and $\rho_{\text {Dish }}^{\text {sat }}$ indicate consumers have substantial heterogeneity in their valuation for satellite bundles (a standard deviation of approximately $\$ 40$ per month); as discussed earlier, such heterogeneity assists the model in matching observed Comcast, DirecTV, and Dish price-cost margins. The implied average predicted margins are given in the second panel of Table 3 .

To illustrate the efficacy of instruments described in the previous section (which include satellite

\footnotetext{
${ }^{54}$ Our estimates can be compared to Goolsbee and Petrin (2004), who estimate household demand for satellite, basic cable, premium cable, and local antenna using 2001 data; they obtain an expanded basic cable own-price elasticity of -1.5 , and an overall satellite own-price elasticity of -2.5. They do not observe cross-sectional variation in prices for satellite distributors, and rely on Slutsky symmetry to identify satellite price elasticities. Our estimated own-price elasticity for cable is similar, and the overall satellite own-price elasticity implied by our own- and cross-price elasticity estimates for DirecTV and Dish, computed at average market shares during our sample period (see Table A.1), is -3.2 .
} 
taxes), the third panel of Table 3 reports the results from a logit demand regression. ${ }^{55}$ Instrumenting for price yields a 22 times larger estimated price coefficient, consistent with the presence of a positive correlation between prices and unobservable bundle characteristics.

The bottom panel of Table 3 reports the semi-elasticity for MVPDs and the outside option given the removal of ESPN from each type of distributor (cable or either of the two satellite providers). For example, the removal of ESPN from DirecTV's bundles implies that its own market share would fall by $19.5 \%$, while those for cable and Dish would increase by $3.9 \%$ and $1.8 \%$ respectively. This implies that for every 1000 households that would leave DirecTV if it lost access to ESPN, 920 would substitute to cable, 67 would substitute to Dish, and 13 would go to the outside option. These types of diversion figures, and in particular those to cable, play a central role in the incentives of an integrated cable provider to deny access to a rival satellite distributor.

\subsection{Pricing, Bargaining, Carriage and Foreclosure Parameters}

We now discuss the parameters contained in $\boldsymbol{\theta}_{3}$ which govern a firm's pricing, bargaining and carriage decisions, as well as our rival foreclosure parameter $\lambda_{R}$.

First, we estimate that the variance of firms' bundle-market-time specific profit shocks $\left(\hat{\sigma}_{\omega}^{2}\right)$ is neither economically nor statistically significant. We estimate that channels capture more than half of the gains from trade when bargaining, although less with integrated distributors $\left(\hat{\zeta}^{I}=0.38\right)$ than non-integrated distributors $\left(\hat{\zeta}^{E}=0.28\right)$.

Our estimated value of $\mu$ indicates that firms internalize a substantial fraction, but not all, of the profits of other integrated units when making decisions. Only $\$ 0.79$ of each dollar of profit realized by its integrated partner is internalized when an integrated MVPD makes pricing and carriage decisions, or when integrated MVPDs and RSNs bargain with each other. (We reject the hypothesis of full internalization at standard confidence levels.)

Our estimated lower bounds for $\mu \times \underline{\lambda}_{R}^{\text {Phil }}$ and $\mu \times \underline{\lambda}_{R}^{S D}$ are 1.11 and 0.94 ; these indicate that integrated channels' supply decisions vis-à-vis non-integrated rival distributors are significantly affected by foreclosure incentives, and these weights placed on the benefits of rival foreclosure for the channel's integrated distributors are not stastically significantly different from $1 .{ }^{56}$ Figure 6

\footnotetext{
${ }^{55}$ For 20,784 firm-market-year bundles, the dependent variable is the log of the ratio of market shares for the bundle to the outside option, and the OLS regressors are firm-state and year fixed effects, channel fixed effects for all channels contained in the bundle, and price. The excluded instruments for price in the IV regression are the satellite tax within the market interacted with an indicator for whether the bundle is offered by a satellite or cable distributor, and the maximum fraction of teams not blacked out within the market across all RSNs for which the market is relevant. The F-statistic on the excluded instruments in the first stage regression of price is 425.4 ; the t-statistic for satellite taxes interacted with a satellite distributor indicator in the first stage price regression is 35; and the $R^{2}$ from the regression is 0.52 . Additionally, an important input into distributor demand elasticities with respect to carriage is the coefficient on mean viewership utility in the distributor choice utility equation in (2). The first stage regression of $v_{f m t}^{*}$ on the same set of instruments for price results in an F-statistic on the excluded instruments of 389.4; the t-statistic for the maximum fraction of teams not blacked out is 33 ; and the $R^{2}$ from the regression is 0.56 .

${ }^{56}$ Given $\hat{\mu}=0.79$, these estimates imply that $\underline{\lambda}_{R}^{\text {Phil }}$ is at least 1.4 and $\underline{\lambda}_{R}^{S D}$ at least 1.3 , which corresponds to the integrated channel placing more weight on its integrated distributor's benefits from foreclosure than the channel and distributor place on each other's profits when pricing, making carriage decisions, and bargaining with each other. However, we cannot reject the hypothesis that either of these values differ from 1: the $95 \%$ confidence interval for
} 


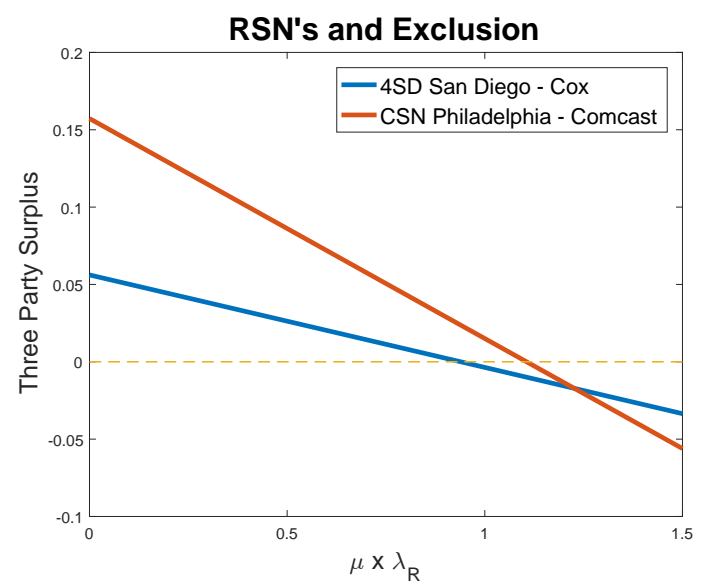

Figure 6: Three-party surplus between the integrated cable MVPD, DirecTV, and Dish as a function of $\mu \times \lambda_{R}$ in Philadelphia and San Diego.

graphs the total three party surplus - given by the left-hand side of (16) - between the integrated channel and the two satellite distributors in the two loophole markets we examine (Philadelphia and San Diego). We see that for values of $\mu \times \lambda_{R}$ lower than 0.94 , it is not an equilibrium for either channel to exclude both satellite distributors as there would be a profitable deviation, for some negotiated set of affiliate fees, for the channel to be supplied. However, for values between approximately 0.94 and 1.11, we can rationalize exclusion in San Diego but not Philadelphia. Only for values of $\mu \times \lambda_{R} \geq 1.11$ does our model rationalize exclusion in both of these loophole markets.

\section{The Welfare Effects of Vertical Integration}

In this section we use our model's estimates to examine how vertical integration affects affiliate fee negotiations (including whether supply occurs at all), distributors' pricing and carriage decisions, and - ultimately - firm and consumer welfare. We focus on 26 RSNs that were active in 2007, 13 of which were (at least partially) integrated with a downstream distributor (10 with a cable MVPD, 3 with DirecTV). ${ }^{57}$ Of these integrated RSNs, two - CSN Philadelphia and 4SD — were owned by cable distributors in "loophole" markets, and were not provided to satellite.

For each of these RSNs we simulate market outcomes for the year 2007 that would have occurred in the RSN's relevant markets under the following three integration scenarios: (1) Non-integration, (2) Integration with PARs, and (3) Integration without PARs. ${ }^{58}$ More specifically:

(i) Non-Integration: In this scenario, we assume that $\mu=0$ and $\lambda_{R}=0$ so that all firms behave $\underline{\hat{\lambda}}_{R_{57}}^{P h i l}$ is $[0.97,1.93]$ and $\underline{\hat{\lambda}}_{R}^{S D}$ is $[0.82,1.70]$.

${ }^{57}$ We exclude from our analysis 3 cable-integrated RSNs (CSN Northwest, Comcast/Charter Sports Southeast, and Cox Sports TV) and one independent RSN (YES) that did not supply satellite providers in markets where PARs were in effect, as our model does not explain this exclusion.

${ }^{58}$ We simulate the equilibrium under all three scenarios for each RSN, including whichever scenario occurred in the data for the RSN. 
as if they are non-integrated (i.e., no MVPD or channel internalizes the profits of any other unit).

(ii) Integration and PARs: In this scenario, if the RSN being examined is non-integrated in the data, we assign full ownership of the channel to the largest cable MVPD in that RSN's relevant markets; if the RSN is integrated, we do not change its ownership structure. We then assume that $\mu$ is equal to our estimated value $\hat{\mu}=0.79$, but that $\lambda_{R}=0$ : i.e., we assume that integrated distributors and channels partially internalize each other's profits when bargaining with each other over affilliate fees, and when the integrated distributor is pricing and making carriage decisions, but that program access rules prevent the channels from considering the benefits of foreclosure to its integrated distributor when bargaining with rival distributors.

(iii) Integration and no-PARs: In our final scenario, we follow the same setup as in the "Integration and PARs" scenario, but assume that $\lambda_{R}=\hat{\lambda}_{R}^{P h i l}$, the larger of our two recovered lower bounds. ${ }^{59}$ The RSN therefore internalizes the profits of its downstream integrated units when bargaining with other downstream distributors, and thus may find it unprofitable to supply downstream rivals.

For each integration scenario and each RSN, we solve for a set of bundle prices, carriage decisions, and negotiated affiliate fees that satisfy the necessary equilibrium conditions given by equations (5), (6), and (11). Under non-integration and integration with PARs (scenarios (i) and (ii)), we assume that all RSNs are supplied to all distributors. ${ }^{60}$ Under scenario (iii), where channels are integrated but PARs are not in effect, we also solve for the RSN's equilibrium supply decision. To determine whether or not each rival distributor is supplied with the channel, we test which supply outcomes (e.g., if a cable integrated RSN supplies both, neither, or either one of the two satellite distributors) are consistent with equilibrium. ${ }^{61}$

\footnotetext{
${ }^{59}$ The value of $\lambda_{R}$ must be at least $\underline{\hat{\lambda}}_{R}^{\text {Phil }}$ to rationalize the non-supply of the satellite distributors that we observe in both Philadelphia and San Diego. If $\lambda_{R}>\underline{\hat{\lambda}}_{R}^{\text {Phil }}$, foreclosure incentives would be larger than those considered here; we explore the effects of larger values of $\lambda_{R}$ in Section 6.3 .

${ }^{60}$ Aside from the two loophole RSNs, all other RSNs in our counterfactuals were provided to all distributors in 2007.

${ }^{61}$ At the set of affiliate fees, prices, and carriage decisions that satisfy the necessary equilibrium conditions under each potential supply outcome, for each cable-owned channel in scenario (iii) we test: (a) whether supplying both satellite providers is an equilibrium by examining if there are positive bilateral gains from trade between the RSN and each satellite provider given that the other satellite provider is supplied; (b) whether supplying only one satellite distributor is an equilibrium by examining if there are positive gains from trade between the RSN and the supplied satellite distributor given that the other satellite provider is not supplied, and if there are no bilateral gains from trade between the RSN and the non-supplied satellite distributor given that the other satellite provider is supplied; and (c) whether supplying neither satellite distributor is an equilibrium by examining if the three-party-surplus given by the left-hand side of (16) is negative. For all RSNs but two, exactly one supply outcome was robust to these tests. For two RSNs, exactly two supply outcomes satisfied these tests: for CSN Philadelphia, they were the non-supply of both satellite distributors and the supply of only DirecTV; for NESN, they were the non-supply of both or supply of both. We report results assuming that the outcome with the least supply is chosen (as this outcome maximized the integrated firms' profits given our parameter estimates). For each of the three RSNs owned by DirecTV, we determine supply by verifying that the bilateral surplus generated by the RSN's supply of each cable MVPD in the RSN's relevant markets as well as Dish Network is positive (where surpluses are computed at updated levels of affiliate fees, prices, and carriage decisions).
} 
In our main counterfactual results, we assume that a change in ownership for a single RSN does not cause national satellite prices to adjust, and we thus hold satellite prices fixed at observed levels. In Section 6.3, we also examine counterfactuals under the alternative assumption that satellite prices are determined at the DMA level, and may adjust across our integration scenarios. Further computational and implementation details are provided in Appendix C.4.

\subsection{Potential Effects}

Before proceeding, it is instructive to highlight the effects of vertical integration that are captured by our model and that we attempt to quantify. Our model emphasizes three main supply-side decisions: (i) negotiations over supply and affiliate fees between channels and distributors, and both (ii) channel carriage (conditional on supply) and (iii) bundle pricing by distributors.

Suppose, then, that MVPD $f$ integrates with RSN $c$, and that there is a rival MVPD $g$. The following effects of vertical integration are admitted in our setting:

1. Bargaining Effects and Foreclosure: When integration occurs there are effects on both internal and external bargaining. As discussed in Section 3.4.3, we expect the effective internal affiliate fee paid by the integrated distributor $f$ to fall when integration occurs: i.e., our 0.79 estimated value of $\mu$ indicates that the RSN and distributor $f$ internalize (most of) each others' payoffs. Of course, in equilibrium, the internal affiliate fee is also affected by any changes in carriage and pricing as these can change both $f$ and $c$ 's gains from trade.

The effects of integration on external bargaining will depend on whether PARs are in effect or not. When PARs are in effect, RSN $c$ ignores any benefits to its integrated distributor $f$ 's profits from denying access to rival distributor $g$, just as if $c$ was not integrated. However, the negotiated affiliate fee to $g$ may still be affected by changes in $f$ 's carriage and prices, which can affect $g$ 's benefit from getting access to $c$, and by any change in the internal affiliate fee that $c$ receives from $f$ (which would alter how supply of $g$ affects $c$ 's profit).

Finally, when $c$ bargains with the rival MVPD $g$ and PARs are not in effect, $c$ internalizes the lost profit of its integrated downstream distributor $f$ if $g$ is supplied (since $\lambda_{R}>0$ ). As a result, the gains-from-trade that accrue to $c$ by supplying $g$ are reduced from what they would be under either non-integration or in the presence of PARs, potentially leading to a higher negotiated affiliate fee $\tau_{g c t}$ or - if gains-from-trade are eliminated altogether-nonsupply. Again, however, any induced changes in carriage and pricing can also affect negotiated input fees.

2. Carriage Effects: When vertical integration occurs, the fact that $\mu$ is positive makes the integrated $f$ internalize the effects of its carriage of $\operatorname{RSN} c$ on $c$ 's profit. As carriage is likely to increase $c$ 's profit due to the increase in affiliate fees earned from $f$ (although an offsetting effect is that f's carriage of $c$ may lower $c$ 's affiliate fee revenues earned from $g$, by reducing

$g$ 's market share), integration may lead $f$ to increase carriage of RSN $c$. The net impact on 
carriage will also depend on equilibrium price adjustments and whether rival distributors are still supplied with the channel.

3. Pricing Effects: As with the carriage decision, an integrated $f$ will internalize effects of its pricing on RSN c's profit. This is likely to push $f$ toward charging a lower bundle price-i.e., reducing double marginalization - as a lower price will increase $f$ 's market share, and hence the affiliate fees that $c$ collects from $f$ (although, as described above, there is a potentially offsetting effect from any reduced affiliate fees earned from $g$, as in Chen 2001). In addition, changes in carriage will have a separate effect on f's pricing; for example, if carriage increases, the resulting increased bundle quality is likely to push $f$ to increase prices in those markets where $c$ is added to its bundle relative to what its prices would have been absent the carriage change.

Thus, while we expect integration to increase carriage and reduce double marginalization by integrated distributors, and the absence of PARs to increase foreclosure of and affiliate fees paid by rival distributors of integrated firms, confounding effects are present that may upset these expectations. Moreover, even if the directions of these effects are as expected, their magnitudes, and their overall impacts on consumer and aggregate welfare remain empirical questions that our counterfactual simulations aim to address.

\subsection{Results}

\subsubsection{Individual RSN Results}

As an illustration of the kinds of effects we see for individual RSNs, Table 4 reports market shares, channel carriage, cable prices, firm profits, and consumer and total welfare across our three different integration scenarios for three RSNs: CSN Philadelphia, a cable-integrated RSN located in a terrestrial loophole market; MSG, a cable-integrated RSN located in a non-loophole market; and NESN, a non-integrated RSN. Below each RSN name is the MVPD that owns the channel (or is assigned ownership under integration scenarios (ii) and (iii) if the RSN is non-integrated, denoted by a * next to the assigned owner's name), the number of households and the MVPD owner's "footprint" (the percentage of these households that the MVPD "passes" or plausibly could serve) in the RSN's relevant markets, and the estimated mean consumer willingness-to-pay (WTP) for the RSN.

The values shown for scenario (i), corresponding to no vertical integration ("No VI"), are household weighted average levels across an RSN's relevant markets; with the exception of market shares and cable carriage, reported numbers are in dollars per household per month. For integration scenarios (ii) and (iii), "VI PARs" and "VI No PARs" respectively, we report changes from non-

integration scenario (i) either as a percentage of non-integration levels (denoted $\% \Delta_{l v l}$ ) or as a percentage of the mean WTP for the RSN (denoted $\% \Delta_{W T P}$ ). A missing value for "Aff Fees to Sat" indicates that the RSN is predicted to be withheld from the two satellite distributors. Confidence 
Table 4: Simulated Market Outcomes for Selected RSNs

\begin{tabular}{|c|c|c|c|c|c|c|}
\hline & & \multirow[t]{2}{*}{ (i) No VI } & \multicolumn{2}{|c|}{ (ii) VI PARs } & \multicolumn{2}{|c|}{ (iii) VI No PARs } \\
\hline & & & \multicolumn{2}{|c|}{ (vs. No VI) } & \multicolumn{2}{|c|}{ (vs. No VI) } \\
\hline & & Level & $\% \Delta_{l v l}$ & $\% \Delta_{W T P}$ & $\% \Delta_{l v l}$ & $\% \Delta_{W T P}$ \\
\hline \multicolumn{7}{|c|}{ CABLE INTEGRATED RSNS } \\
\hline CSN PHIL & Cable Mkt Share & 0.64 & $0.8 \%$ & & $1.8 \%$ & \\
\hline Comcast & & {$[0.62,0.65]$} & {$[0.2 \%, 2.4 \%]$} & & {$[0.6 \%, 4.0 \%]$} & \\
\hline Pop $4.25 \mathrm{M}$ & Sat Mkt Share & 0.18 & $-0.5 \%$ & & $-10.4 \%$ & \\
\hline Footprint $90 \%$ & & {$[0.17,0.19]$} & {$[-3.3 \%,-0.2 \%]$} & & {$[-14.8 \%,-0.5 \%]$} & \\
\hline WTP $\$ 4.99$ & Cable Carriage & 0.95 & $1.6 \%$ & & $0.4 \%$ & \\
\hline \multirow{13}{*}{ Foreclose: $85 \%$} & & {$[0.62,0.97]$} & {$[0.0 \%, 53.8 \%]$} & & {$[-6.2 \%, 52.9 \%]$} & \\
\hline & Cable Prices & 54.31 & $-0.5 \%$ & & $0.9 \%$ & \\
\hline & & {$[53.28,55.42]$} & {$[-1.5 \%, 0.9 \%]$} & & {$[-1.4 \%, 1.8 \%]$} & \\
\hline & Aff Fees to Sat & 2.26 & $3.6 \%$ & & - & \\
\hline & & {$[1.00,2.64]$} & {$[-9.4 \%, 7.0 \%]$} & & - & \\
\hline & Cable + RSN Surplus & 30.19 & $0.2 \%$ & $0.9 \%$ & $1.1 \%$ & $6.5 \%$ \\
\hline & & {$[14.57,32.67]$} & {$[0.0 \%, 2.4 \%]$} & {$[0.3 \%, 13.7 \%]$} & {$[0.4 \%, 3.3 \%]$} & {$[3.0 \%, 20.5 \%]$} \\
\hline & Satellite Surplus & 4.29 & $-0.9 \%$ & $-0.8 \%$ & $-2.1 \%$ & $-1.8 \%$ \\
\hline & & {$[1.26,4.70]$} & {$[-3.4 \%,-0.4 \%]$} & {$[-2.4 \%,-0.5 \%]$} & {$[-4.8 \%,-1.1 \%]$} & {$[-4.5 \%,-0.9 \%]$} \\
\hline & Consumer Welfare & 31.21 & $0.6 \%$ & $3.9 \%$ & $-2.9 \%$ & $-18.1 \%$ \\
\hline & & {$[16.82,34.81]$} & {$[0.2 \%, 2.0 \%]$} & {$[1.4 \%, 12.7 \%]$} & {$[-3.3 \%, 1.5 \%]$} & {$[-21.8 \%, 9.9 \%]$} \\
\hline & Total Welfare & 65.69 & $0.3 \%$ & $4.0 \%$ & $-1.0 \%$ & $-13.4 \%$ \\
\hline & & {$[31.14,71.73]$} & {$[0.1 \%, 1.9 \%]$} & {$[2.0 \%, 25.2 \%]$} & {$[-1.1 \%, 1.1 \%]$} & {$[-15.6 \%, 14.7 \%]$} \\
\hline MSG & Cable Mkt Share & 0.63 & $3.3 \%$ & & $3.3 \%$ & \\
\hline Cablevision & & {$[0.62,0.67]$} & {$[0.3 \%, 4.8 \%]$} & & {$[0.2 \%, 4.7 \%]$} & \\
\hline Pop $11.7 \mathrm{M}$ & Sat Mkt Share & 0.18 & $-4.3 \%$ & & $-4.3 \%$ & \\
\hline Footprint $42 \%$ & & {$[0.17,0.18]$} & {$[-7.1 \%,-0.4 \%]$} & & {$[-8.1 \%,-0.4 \%]$} & \\
\hline \multirow[t]{4}{*}{ Pred WTP $\$ 2.32$} & Cable Carriage & 0.68 & $10.5 \%$ & & $10.5 \%$ & \\
\hline & & {$[0.67,0.87]$} & {$[-2.5 \%, 18.5 \%]$} & & {$[-3.1 \%, 18.5 \%]$} & \\
\hline & Cable Prices & 59.40 & $-2.4 \%$ & & $-2.4 \%$ & \\
\hline & & {$[56.80,60.81]$} & {$[-3.5 \%, 0.0 \%]$} & & {$[-3.5 \%, 0.2 \%]$} & \\
\hline \multirow{10}{*}{ Foreclose: $1 \%$} & Aff Fees to Sat & 1.22 & $-3.3 \%$ & & $22.4 \%$ & \\
\hline & & {$[0.42,1.28]$} & {$[-5.9 \%, 10.4 \%]$} & & {$[17.1 \%, 53.4 \%]$} & \\
\hline & Cable + RSN Surplus & 30.64 & $0.3 \%$ & $4.4 \%$ & $0.5 \%$ & $6.8 \%$ \\
\hline & & {$[14.61,34.12]$} & {$[-0.1 \%, 0.6 \%]$} & {$[-1.6 \%, 7.4 \%]$} & {$[0.0 \%, 1.3 \%]$} & {$[0.4 \%, 14.6 \%]$} \\
\hline & Satellite Surplus & 4.16 & $-4.2 \%$ & $-7.5 \%$ & $-5.5 \%$ & $-9.9 \%$ \\
\hline & & {$[1.24,4.48]$} & {$[-7.2 \%,-0.5 \%]$} & {$[-12.1 \%,-0.9 \%]$} & {$[-8.5 \%,-1.2 \%]$} & {$[-14.3 \%,-2.4 \%]$} \\
\hline & Consumer Welfare & 33.80 & $3.1 \%$ & $44.6 \%$ & $3.0 \%$ & $44.3 \%$ \\
\hline & & {$[18.38,38.14]$} & {$[0.3 \%, 4.3 \%]$} & {$[4.4 \%, 66.3 \%]$} & {$[-0.4 \%, 4.3 \%]$} & {$[-6.3 \%, 66.0 \%]$} \\
\hline & Total Welfare & 68.60 & $1.4 \%$ & $41.4 \%$ & $1.4 \%$ & $41.2 \%$ \\
\hline & & {$[32.06,76.01]$} & {$[0.1 \%, 1.9 \%]$} & {$[3.4 \%, 60.9 \%]$} & {$[0.1 \%, 1.9 \%]$} & {$[2.5 \%, 60.7 \%]$} \\
\hline \multicolumn{7}{|c|}{ NON-INTEGRATED RSN } \\
\hline NESN & Cable Mkt Share & 0.61 & $7.6 \%$ & & $9.4 \%$ & \\
\hline${ }^{*}$ Comcast & & {$[0.59,0.65]$} & {$[1.6 \%, 11.2 \%]$} & & {$[2.7 \%, 12.5 \%]$} & \\
\hline Pop 5.20M & Sat Mkt Share & 0.13 & $-7.8 \%$ & & $-22.3 \%$ & \\
\hline Footprint $85 \%$ & & {$[0.12,0.14]$} & {$[-12.6 \%,-1.8 \%]$} & & {$[-26.5 \%,-7.2 \%]$} & \\
\hline \multirow[t]{4}{*}{ WTP $\$ 6.91$} & Cable Carriage & 0.92 & $6.2 \%$ & & $3.6 \%$ & \\
\hline & & {$[0.68,0.98]$} & {$[0.0 \%, 33.1 \%]$} & & {$[-0.5 \%, 38.1 \%]$} & \\
\hline & Cable Prices & 56.73 & $-4.7 \%$ & & $-3.9 \%$ & \\
\hline & & {$[54.24,57.88]$} & {$[-6.6 \%,-0.5 \%]$} & & {$[-6.0 \%, 0.6 \%]$} & \\
\hline \multirow[t]{10}{*}{ Foreclose: $96 \%$} & Aff Fees to Sat & 3.32 & $3.1 \%$ & & - & \\
\hline & & {$[1.23,3.79]$} & {$[-12.6 \%, 16.9 \%]$} & & - & \\
\hline & Cable + RSN Surplus & 28.38 & $0.9 \%$ & $3.6 \%$ & $2.0 \%$ & $8.2 \%$ \\
\hline & & {$[13.68,31.36]$} & {$[0.1 \%, 2.4 \%]$} & {$[0.9 \%, 10.6 \%]$} & {$[0.7 \%, 4.0 \%]$} & {$[5.4 \%, 16.7 \%]$} \\
\hline & Satellite Surplus & 2.96 & $-8.3 \%$ & $-3.5 \%$ & $-10.9 \%$ & $-4.7 \%$ \\
\hline & & {$[0.84,3.24]$} & {$[-13.2 \%,-1.8 \%]$} & {$[-5.5 \%,-1.3 \%]$} & {$[-13.9 \%,-3.0 \%]$} & {$[-6.3 \%,-1.7 \%]$} \\
\hline & Consumer Welfare & 28.36 & $6.4 \%$ & $26.5 \%$ & $3.3 \%$ & $13.5 \%$ \\
\hline & & {$[15.54,31.97]$} & {$[1.4 \%, 10.0 \%]$} & {$[8.2 \%, 40.8 \%]$} & {$[-1.7 \%, 7.1 \%]$} & {$[-9.0 \%, 29.2 \%]$} \\
\hline & Total Welfare & 59.70 & $3.1 \%$ & $26.5 \%$ & $2.0 \%$ & $17.0 \%$ \\
\hline & & {$[29.79,65.84]$} & {$[0.5 \%, 5.1 \%]$} & {$[7.8 \%, 43.7 \%]$} & {$[-0.2 \%, 4.5 \%]$} & {$[-2.5 \%, 37.5 \%]$} \\
\hline
\end{tabular}

Notes: Scenarios (i)-(iii) correspond to the integration scenarios described at the beginning of Section 6 . Beneath the RSN name is either the name of the RSN's owner (observed or, if non-integrated, assigned, which is denoted by ${ }^{*}$ ), the number of television households in the RSN's relevant markets, the MVPD owner's footprint (\% of households passed) in the RSN's relevant markets, and the estimated mean consumer WTP for the RSN. Scenario (i) reports household weighted averages over all relevant markets for each RSN, where all levels except for market shares and cable carriage are in $\$ /$ household $/$ month. Scenarios (ii) and (iii) report changes from scenario (i), where $\% \Delta_{l v l}$ (respectively, $\% \Delta_{W T P}$ ) represent changes from scenario (i) expressed as a percentage of changes in household weighted averages of levels (respectively, estimated mean consumer WTP for the channel). Affiliate fees to satellite are reported conditional on supply; missing values indicate foreclosure. 95\% confidence intervals, constructed from 150 simulations, are reported below each figure; the fraction of simulations in which the RSN is predicted to foreclose at least one rival distributor under scenario (iii) is reported last under each RSN's name ("Foreclose: \%"). Results for all other RSNs are contained in Appendix Tables A.5-A.10. 
Table 5: Average Simulated Market Outcomes Across All RSNs

\begin{tabular}{|c|c|c|c|c|c|c|c|}
\hline & \multirow[t]{2}{*}{ (i) No VI } & \multicolumn{2}{|c|}{ (ii) VI PARs } & \multicolumn{4}{|c|}{ (iii) VI No PARs } \\
\hline & & \multicolumn{2}{|c|}{ (vs. No VI) } & \multicolumn{2}{|c|}{ (vs. No VI) } & \multicolumn{2}{|c|}{ (vs. VI PARs) } \\
\hline & Level & $\% \Delta_{l v l}$ & $\% \Delta_{W T P}$ & $\% \Delta_{l v l}$ & $\% \Delta_{W T P}$ & $\% \Delta_{l v l}$ & $\% \Delta_{W T P}$ \\
\hline \multicolumn{8}{|l|}{ ALL RSNS } \\
\hline \multirow[t]{2}{*}{ Cable Mkt Share } & 0.60 & $2.1 \%$ & & $2.2 \%$ & & $0.1 \%$ & \\
\hline & {$[0.59,0.62]$} & {$[0.4 \%, 2.6 \%]$} & & {$[0.4 \%, 2.6 \%]$} & & {$[-0.2 \%, 0.3 \%]$} & \\
\hline \multirow{2}{*}{ Sat Mkt Share } & 0.20 & $-2.0 \%$ & & $-2.7 \%$ & & $-0.8 \%$ & \\
\hline & {$[0.20,0.21]$} & {$[-2.6 \%,-0.4 \%]$} & & {$[-4.1 \%,-0.8 \%]$} & & {$[-2.6 \%,-0.0 \%]$} & \\
\hline \multirow[t]{2}{*}{ Cable Carriage } & 0.72 & $9.4 \%$ & & $8.6 \%$ & & $-0.7 \%$ & \\
\hline & {$[0.66,0.80]$} & {$[3.1 \%, 21.5 \%]$} & & {$[0.8 \%, 19.1 \%]$} & & {$[-4.4 \%, 0.9 \%]$} & \\
\hline \multirow[t]{2}{*}{ Cable Prices } & 55.10 & $-1.2 \%$ & & $-1.1 \%$ & & $0.1 \%$ & \\
\hline & {$[54.25,55.90]$} & {$[-1.5 \%,-0.1 \%]$} & & {$[-1.4 \%,-0.0 \%]$} & & {$[0.0 \%, 0.3 \%]$} & \\
\hline Aff Fees to Rivals $(a)$ & $\begin{array}{r}1.36 \\
{[0.54 .1 .45]}\end{array}$ & $\begin{array}{r}-0.7 \% \\
{[-3.2 \% .4 \%]}\end{array}$ & & $\begin{array}{r}17.1 \% \\
{[11.0 \% .28 .5 \%]}\end{array}$ & & $\begin{array}{r}18.0 \% \\
{[12.1 \% .28 .6 \%]}\end{array}$ & \\
\hline \multirow[t]{2}{*}{ Cable + RSN Surplus } & 23.04 & $0.3 \%$ & $3.1 \%$ & $0.6 \%$ & $5.0 \%$ & $0.2 \%$ & $1.9 \%$ \\
\hline & {$[11.13,25.17]$} & {$[0.1 \%, 0.6 \%]$} & {$[1.4 \%, 5.4 \%]$} & {$[0.2 \%, 0.9 \%]$} & {$[2.6 \%, 8.1 \%]$} & {$[0.1 \%, 0.5 \%]$} & {$[0.5 \%, 4.2 \%]$} \\
\hline \multirow[t]{2}{*}{ Satellite Surplus } & 5.10 & $-2.2 \%$ & $-4.3 \%$ & $-3.2 \%$ & $-6.0 \%$ & $-1.0 \%$ & $-1.7 \%$ \\
\hline & {$[1.56,5.51]$} & {$[-3.1 \%,-0.5 \%]$} & {$[-6.7 \%,-0.3 \%]$} & {$[-3.9 \%,-1.0 \%]$} & {$[-8.4 \%,-1.1 \%]$} & {$[-1.3 \%,-0.4 \%]$} & {$[-2.5 \%,-0.6 \%]$} \\
\hline \multirow[t]{2}{*}{ Consumer Welfare } & 30.99 & $1.5 \%$ & $18.0 \%$ & $1.3 \%$ & $16.2 \%$ & $-0.2 \%$ & $-1.7 \%$ \\
\hline & {$[16.15,34.47]$} & {$[0.3 \%, 1.8 \%]$} & {$[5.5 \%, 23.8 \%]$} & {$[-0.1 \%, 1.5 \%]$} & {$[-1.3 \%, 20.4 \%]$} & {$[-0.7 \%,-0.1 \%]$} & {$[-8.4 \%, 0.0 \%]$} \\
\hline \multirow[t]{2}{*}{ Total Welfare } & 59.13 & $0.7 \%$ & $16.8 \%$ & $0.6 \%$ & $15.3 \%$ & $-0.1 \%$ & $-1.5 \%$ \\
\hline & {$[27.59,64.41]$} & {$[0.1 \%, 0.9 \%]$} & {$[5.4 \%, 22.2 \%]$} & {$[0.0 \%, 0.8 \%]$} & {$[0.8 \%, 18.7 \%]$} & {$[-0.2 \%,-0.0 \%]$} & {$[-6.4 \%, 0.1 \%]$} \\
\hline \# Foreclosed: & & & & $4 / 26[0,9]$ & & & \\
\hline
\end{tabular}

Notes: Average simulated market outcomes across the 26 RSNs in our analysis, weighted by the number of households in each RSN's relevant markets. Percentages are the averages of percentage changes across RSNs, weighted by the number of households in each RSN's relevant markets. "\# Foreclosed" reports the number of RSNs that are not provided to rival distributors under (iii) VI No PARs. See Table 4 and main text for additional details.

(a) "Fees to Rival" represents average affilliate fees (to the satellite MVPDs for cable-integrated RSNs, and to cable MVPDs and the rival satellite distributor for satellite-integrated RSNs) conditional on supply in each relevant scenario.

intervals are constructed by taking 150 draws from the joint distribution of the estimated coefficients and re-computing the equilibrium for each draw. The fraction of the scenario (iii) draws for which the RSN is predicted to foreclose and not supply at least one rival distributor is also shown last under each RSN name ("Foreclose: \%").

For each of the three RSNs shown in Table 4 vertical integration with PARs in effect leads the integrated distributor to increase carriage and reduce its bundle price at our point estimates, and in each case we can reject a zero effect for at least one of the carriage or price effects. Cable's market share increases in each case, and satellite's share decreases; as well, satellite surplus falls, consumer welfare increases, and total welfare rises (all such effects are statistically significant).

When integration occurs without PARs in effect, two of these three RSNs - CSN Philadelphia and NESN - deny access to both rival satellite producers. Despite this exclusion, only for CSN Philadelphia are the point estimates for the effects of vertical integration on consumer and total welfare negative, and for neither of these two RSNs can we reject zero net consumer and total welfare effects. For the third RSN, MSG, the two satellite distributors continue to have access to the RSN, although paying higher affiliate fees $(22.4 \%$ higher according to our point estimates, and statistically significantly different from zero). For all three RSNs, vertical integration without PARs in effect lowers the satellite distributors' profits by between 2-11\% (statistically significant in each case).

Results for all other RSNs are contained in Appendix Tables A.5-A.10. Overall, the outcomes for different RSNs display considerable heterogeneity. 


\subsubsection{Average Results}

We now turn to the average effects of vertical integration. Table 5 reports market outcomes for each of the three vertical integration scenarios, averaged across RSNs and weighted by the number of households in each RSN's relevant markets. The structure is the same as in Table 4, with the following adjustments. First, "Aff Fees to Rivals" represents the affiliate fees charged to the integrated MVPD's rival distributors, conditional on the channel being supplied to those distributors. These rival distributors are the two satellite distributors if the channel is cableintegrated; if instead the channel is DirecTV-integrated, these rivals are the cable distributors in the RSN's relevant markets and Dish. Second, "\# Foreclosed" represents the number of RSNs that are not provided to at least one rival distributor for the case of integration without PARs in effect (integration scenario (iii)). Third, in the rightmost two columns, we report the weighted average change in predicted outcomes between scenarios (ii) and (iii); these changes are expressed both as percentages of scenario (ii) levels and of an RSN's estimated mean WTP, and isolate the impact of program access rules given integration. Finally, percentages are the averages of percentage changes across RSNs, weighted by the number of households in each RSN's relevant markets.

Efficiency Effects: Reduction of Double Marginalization and Increased Carriage. We first focus on the potential efficiency gains from vertical integration. These are highlighted by the differences between integration scenario (ii) with PARs and non-integration scenario (i), reported in the second and third columns of Table 5 .

Across all RSNs, we predict that integration of a single RSN when PARs are in effect yields on average a (statistically significant) $9.4 \%$ increase in carriage of the RSN by cable distributors. ${ }^{62}$ It also results on average in a (statistically significant) $1.2 \%$ decrease in cable prices (corresponding to an average $\$ 0.67$ reduction in the price consumers pay). ${ }^{63,64}$ As discussed in Section 6.1 , pricing reductions arise primarily from the reduction of double marginalization. However, there are offsetting effects that may mitigate downward pricing incentives: integrated distributors now internalize affiliate fees paid by rival MVPDs, and (as we have noted) carriage of the RSN by cable providers increases when the channel is integrated (thereby increasing the utility delivered by bundles in certain markets). Even so, cable prices fall on average. ${ }^{65}$

We find that joint RSN and integrated cable surplus increases on average when moving from non-integration to integration with PARs: when a cable MVPD is integrated (and since $\hat{\mu}$ is greater than 0), its pricing decisions will partially internalize RSN profits (even if, under PARs, the channel

\footnotetext{
${ }^{62}$ This average includes carriage changes by cable operators for the three satellite-owned RSNs.

${ }^{63}$ The values reported for scenarios (ii) and (iii) in Tables 4-5 are the (household weighted) averages of percentage changes, not the percentage change in the average levels. Thus, the average $\$ 0.67$ decrease in price that we describe here does not equal the product of the values in scenarios (i) and (ii) in Table 5.

${ }^{64}$ Though integration of most RSNs yields less than a $1 \%$ decrease in cable prices, there are several cases where price decreases are much larger: e.g., integrating NESN with Comcast, reported in the bottom panel of Table 4, results in average cable prices falling by nearly $5 \%$ (corresponding to an average reduction in the price consumers pay of $\$ 2.67$ ) due to NESN's high estimated affiliate fees to Comcast (predicted to be approximately $\$ 4.70$ per month).

${ }^{65} \mathrm{In}$ fact, in our individual RSN results average cable prices do not increase for any RSN in the presence of PARs.
} 
Table 6: Welfare Changes From Foreclosure

\begin{tabular}{lll}
\hline \hline \multicolumn{3}{c}{ Percentage change in levels between (ii) VI PARs and (iii) VI No PARs } \\
\hline & $\% \Delta$ Consumer Welfare & $\% \Delta$ Total Welfare \\
\hline Are Rival Distributors Excluded & $-1.95^{* * *}$ & $-0.72^{* * *}$ \\
& $(0.53)$ & $(0.22)$ \\
\hline $\mathrm{N}$ & 3900 & 3900 \\
$R^{2}$ & 0.52 & 0.52 \\
\hline
\end{tabular}

Notes: Regression where the dependent variable is the percentage change in either consumer or total welfare in levels between integration scenarios (ii) and (iii) (with and without PARs in effect). Each observation is an RSNcounterfactual simulation $(26 \times 150)$. Specifications include RSN fixed effects. Standard errors clustered at RSN level, and ${ }^{* * *}$ represents significance at the $1 \%$ level.

does not act upon rival foreclosure incentives). Satellite surplus, on average across all RSNs, falls by $2.2 \%$ when RSNs are integrated with PARs in effect. ${ }^{6}$ Consumer welfare and total welfare increase by, on average, $1.5 \%$ and $0.7 \%$ respectively ( $18.0 \%$ and $16.8 \%$, respectively, as percentage gains of WTP for the RSN). The change in total welfare represents an average increase of $\$ 0.43$ per household per month. Each of these changes is statistically significant.

Foreclosure Effects: Raising Rivals' Costs and Exclusion. The comparison of scenarios (ii) and (iii), shown in the last two columns of Table 5 provides the impact of removing PARs given that RSNs are integrated, and isolates the impact of foreclosure incentives on market outcomes. Allowing for foreclosure is predicted to reduce both consumer and total welfare from the integration scenario with PARs by $0.2 \%$ and $0.1 \%$, respectively; both are statistically significant, and represent average changes of $1.7 \%$ and $1.5 \%$ as a percentage of the WTP for the RSN.

The reduction in welfare from the absence of PARs stems primarily from two effects. The first occurs when an RSN is completely withheld from rival MVPDs. Though we predict that none of the three DirecTV-owned RSNs would choose to exclude cable providers, we predict that 3 out of the 14 RSNs integrated with a cable provider in the data (the two loophole RSNs and CSN New England) and one previously non-integrated RSN (NESN) would exclude both satellite distributors. ${ }^{67}$ Conditional on integration occurring, exclusion of a rival distributor is associated with a negative change in welfare: Table 6 reports results from a regression of the change in consumer and total welfare between VI scenarios with and without PARs on whether or not rival distributors are denied access to the RSN. Results indicate that the exclusion of rival distributors is associated with a $1.9 \%$ and $0.7 \%$ reduction in consumer and total welfare, which roughly equals the predicted average welfare gains from integration with PARs $\left(\% \Delta_{l v l}\right.$ in scenario (ii)).

To examine when exclusion is more likely to occur, Table 7 reports results from a linear probability regression of whether rival distributors are denied access to an RSN when PARs are not in effect; the footprint, or percentage of households in the RSN's relevant markets that the integrated distributor can serve, is positive and statistically significant. This is consistent with the discussion

\footnotetext{
${ }^{66}$ This percentage includes both cable and satellite integration of RSNs, although it is primarily reflecting cable ownership of RSNs. In Appendix Table A.7, we report market outcomes for the three satellite integrated RSNs.

${ }^{67}$ The $95 \%$ confidence interval for the number of RSNs that exclude is $[0,9]$.
} 
Table 7: Probability of Exclusion

\begin{tabular}{|c|c|}
\hline \multicolumn{2}{|c|}{ Exclusion of Rival Distributors by Integrated RSN (Without PARs) } \\
\hline Footprint of Integrated Owner & $\begin{array}{l}0.67^{* *} \\
(0.25)\end{array}$ \\
\hline WTP for RSN & $\begin{array}{l}0.07 \\
(0.05)\end{array}$ \\
\hline $\mathrm{N}$ & 26 \\
\hline$R^{2}$ & 0.29 \\
\hline
\end{tabular}

Notes: Linear probability regression where the dependent variable is whether rival distributors are denied access to an RSN under integration scenario (iii) without PARs. Each observation is an RSN. Specification includes a fixed effect for whether the RSN owner is a cable operator. ${ }^{* *}$ represents significance at the $5 \%$ level.

in Section 4.2 (i.e., larger cable footprints increase the potential losses incurred by an integrated cable provider from supplying the RSN to rival satellite distributors), and reflects the fact that the cable owners for the four RSNs that are predicted to foreclose satellite distributors all have greater than an $85 \%$ footprint.

The second effect that reduces welfare arises when an integrated RSN still supplies rival distributors but raises their affiliate fees, which in turn affects downstream distributor pricing and carriage. Table 5 indicates that affiliate fees for integrated RSNs charged to rivals, conditional on supply, increase on average by a statistically significant $18.0 \%$ from the levels predicted when PARs are in effect. ${ }^{68}$ Even though we have assumed that satellite distributors do not adjust their prices in our counterfactuals, higher satellite affiliate fees can negatively harm consumer welfare by inducing the integrated cable owner to increase its own downstream prices or reduce its carriage. Intuitively, if a cable-integrated RSN increases its affiliate fees with satellite distributors, then the RSN's downstream cable MVPD sees a greater benefit to its integrated RSN from raising its price or reducing its carriage, which will move subscribers to the now higher-paying satellite distributors. Indeed, we find that cable prices increase, on average, by a small, but statistically significant $0.1 \%$; carriage falls in our point estimates by $0.7 \%$, although this change is not statistically significant. ${ }^{69}$ These changes also further reduce satellite firms' surplus by a statistically significant $1.0 \%$ beyond the reduction caused by integration when PARs are in effect.

Net Effects. The comparison of scenarios (iii) and (i), shown in the fourth and fifth columns of Table 5, gives the overall net impact of integration of RSNs when PARs are not in effect. On average across all RSNs, the efficiency effects dominate the foreclosure effects when examining consumer and total welfare - both increase by approximately $1.3 \%$ and $0.6 \%$, representing $15-16 \%$ of the total WTP generated by an RSN. ${ }^{70}$ This is driven by an increase in RSN carriage (9\% on average) and a reduction in cable prices (1.1\%). However, satellite market shares and profits are

\footnotetext{
${ }^{68}$ In some cases this increase represents an increase of nearly $\$ 0.50$ per month per subscriber, as with CSN MidAtlantic (see Table A.6).

${ }^{69}$ This effect is discussed in Chen (2001). As a specific example, Comcast, the owner of CSN Mid-Atlantic, increases its own price of a bundle by $\$ 0.11$ between scenarios (ii) and (iii) as a result of negotiating a significantly higher affiliate fee for CSN Mid-Atlantic from satellite distributors.

${ }^{70}$ On average, consumer welfare rises by $\$ 0.39$ and total welfare by $\$ 0.38$ per household per month.
} 
predicted to fall by $2.7 \%$ and $3.2 \%$, respectively, when RSNs are integrated. ${ }^{71}$

Looking separately at the 4 markets in which exclusion occurs at our point estimates, consumer and total welfare increase on average by small amounts ( $0.4 \%$ and $0.3 \%$ respectively) and we cannot reject zero effects. In contrast, consumer and total welfare rise on average by a statistically significant $1.4 \%$ and $0.7 \%$ respectively in the 22 markets in which exclusion does not occur.

\subsection{Robustness}

Internalization and Rival Foreclosure Parameters. We conducted our counterfactuals under the assumption that our rival foreclosure parameter $\lambda_{R}$ equals the largest estimated lower bound across the two terrestrial loophole RSNs. However, it may be that this bound is not binding, and the value of $\lambda_{R}$ is much larger. To understand the sensitivity of our results to this assumption, we have compared the average percentage change in consumer and total welfare between integration without PARs and no integration (scenarios (iii) and (i)) for different values of $\lambda_{R}$ at our estimated parameter values. Under our main specification when $\lambda_{R}=\underline{\hat{\lambda}}_{R}^{\text {Phil }}=1.4$, the predicted average changes in consumer and total welfare are $1.3 \%$ and $0.6 \%$; at $\lambda_{R}=2$, they are $0.4 \%$ and $0.3 \%$, and at $\lambda_{R}=3$, they are $-0.2 \%$ and $-0.03 \%$. Thus, our rival foreclosure parameter would need to be significantly larger than our estimated lower bounds for the predicted overall average welfare effects from vertical integration (without PARs) to be reversed.

We also have examined the sensitivity of our results to the assumption that internalization is imperfect, and recomputed our counterfactual simulations when $\mu=\lambda_{R}=1 .^{72}$ Our findings are broadly similar: again, examining average changes between integration without PARs and no integration (scenarios (iii) and (i)), we find that consumer and total welfare gains are slightly larger $(1.5 \%$ and $0.7 \%)$; this is attributable to larger increases in carriage $(11.5 \%)$ and reductions in cable prices $(-1.3 \%)$. These differences are not statistically significant.

Satellite Re-Pricing. Our consideration of one vertical merger or divestiture at a time motivated our holding satellite distributors' prices fixed in our primary counterfactual specification-i.e., as each RSN is only active in a subset of markets, changes brought on by adjustments in its ownership might not warrant a change in either satellite distributor's national prices. However, were integration to increase nationally and lead to foreclosure or higher affiliate fees charged to satellite distributors in many markets, we may expect satellite prices to adjust, thereby altering our predicted welfare effects. To address this concern, we repeat our counterfactual simulations under the alternative assumption that satellite prices for both DirecTV and Dish are chosen at the

\footnotetext{
${ }^{71}$ These average net welfare changes mask considerable heterogeneity in the point estimates for individual RSNs. For instance, we find that foreclosure effects dominate for consumer welfare in the terrestrial loophole markets, where 4SD and CSN Philadelphia are predicted to exclude both satellite distributors, and reduce consumer welfare by $8-18 \%$ (\$0.13-0.90) of the average WTP generated by the channel. On the other hand, average net consumer welfare gains from integration for some RSNs reach approximately $\$ 1$ per household per month (e.g., $\$ 1.03$ for MSG and $\$ 0.94$ for NESN).

${ }^{72}$ We conduct these exercises at our estimated parameters, only varying $\mu$ and $\lambda_{R}$; we do not re-estimate our model under the assumption that $\mu=1$. For these exercises, we also assume that $\mu=0.99$ so that internal negotiated affiliate fees are not indeterminate when $O_{f c t}=1$ (see footnote 36 ).
} 
DMA (as opposed to national) level, and can adjust across integration scenarios (see Appendix C.4 for implementation details).

In Appendix Table A.11, we report market outcomes averaged across all RSNs from these additional simulations. When satellite prices are allowed adjust, they fall on average by approximately $1.0 \%$ when RSNs are integrated and PARs are not in effect; compared to the predicted changes when satellite prices are held fixed (Table 5), the negative impact on satellite market shares and surplus is slightly mitigated, as is the increase in negotiated affiliate fees (e.g., when moving from non-integration to VI without PARs, affiliate fees to rivals increase by $16.5 \%$ on average as opposed to by $17.1 \%$ when satellite prices are held fixed). Nevertheless, none of the predicted levels or changes are statistically different from the case in which satellite prices are held fixed. In addition, our main findings also do not change.

\section{Concluding Remarks}

In this paper, we have developed a framework for the analysis of vertical integration and mergers, and applied it to examine the welfare effects of - and regulatory policy regarding-vertical integration of high value sports content in the U.S. cable and satellite television industry. The framework accounts for consumer viewership and subscription decisions, distributor pricing and carriage decisions, and channel-distributor bargaining over affiliate fees. Most importantly, it allows for vertical integration to reduce double marginalization and increase carriage as well as result in foreclosure of rivals from integrated content or raise their costs of carriage. We also allow for imperfect internalization of incentives across integrated divisions within a firm.

Our main results are as follows: (i) vertical integration leads to welfare gains when program access rules are effectively enforced; (ii) failure to effectively enforce program access rules for integrated RSNs leads to consumer and total welfare losses; (iii) in the absence of program access rules, predicted efficiency effects of vertical integration outweigh foreclosure effects on average, resulting in net consumer and total welfare increases compared to non-integration; (iv) welfare gains from vertical integration in the absence of program access rules appear to be largely absent in cases in which exclusion of rival distributors occurs, but positive when rivals continue to have access to the RSN; and (v) rival distributors are harmed when an RSN becomes integrated.

As we have noted previously, our analysis is partial and can be extended in a number of directions. First, our model has focused on comparing the efficiency effects of vertical integration to potential foreclosure of downstream distributors by integrated channels, and does not examine the foreclosure of "upstream" rival channels by an integrated distributor. ${ }^{73}$ Second, investment effects - both on the part of content providers and distributors in channel, programming, and distribution service quality - may change upon integration (Grossman and Hart, 1986; Bolton and

\footnotetext{
${ }^{73}$ See, e.g., Waterman and Weiss (1996) who provide reduced form evidence that integration reduces carriage of rival channels. This finding could be due to foreclosure, but could also arise simply because the increased carriage of the integrated channel makes carriage of the rival channel less attractive. Our model does include the possibility of the latter effect, but does not incorporate any mechanism that might cause foreclosure of rival channels, such as an impact on the integrated channel's advertising revenues.
} 
Whinston, 1991; Hart, 1995), and are absent from the current study. As noted in the introduction, the impact of such investment effects on welfare is ambiguous, and is the subject of future work. Our estimates and counterfactual exercises also relied on a number of modeling assumptions regarding bargaining, the form of affiliate fee pricing, and the effects of program access rule enforcement that could usefully be further examined. Finally, incorporating additional responses to vertical integration, examining how predictions might be impacted by weakened information sharing or misalignment of incentives within the firm, and documenting and measuring the strength of these vertical integration effects in other industries remain promising areas for future research. 


\section{References}

Ackerberg, Daniel A. (2009). 'A New Use of Importance Sampling to Reduce Computational Burden in Simulation Estimation', Quantitative Marketing and Economics 7(4), 343-376.

Asker, John. (2016). 'Diagnosing Foreclosure due to Exclusive Dealing', Journal of Industrial Economics 64(3), 375-410.

Azar, Jose, Schmalz, Martin C. and Tecu, Isabel. (2016), Anti-Competitive Effects of Common Ownership. Unpublished.

Berry, Steven, Levinsohn, James and Pakes, Ariel. (1995). 'Automobile Prices in Market Equilibrium', Econometrica 63(4), 841-890.

Bolton, Patrick and Whinston, Michael D. (1991). 'The "Foreclosure" Effects of Vertical Mergers', Journal of Institutional and Theoretical Economics 147, 207-226.

Bresnahan, Timothy F. and Salop, Steven C. (1986). 'Quantifying the Competitive Effects of Production Joint Ventures', International Journal of Industrial Organization 4, 155-175.

Bresnahan, Timothy and Levin, Jonathan. (2013), Vertical Integration and Market Structure, in Robert Gibbons and John Roberts., eds, 'Handbook of Organizational Economics', Princeton University Press, Princeton, NJ.

Caves, Kevin W., Holt, Chris C. and Singer, Hal J. (2013). 'Vertical Integration in Multichannel Television Markets: A Study of Regional Sports Networks', Review of Network Economics 12(1), 61-92.

Chen, Dong and Waterman, David. (2007). 'Vertical Ownership, Program Network Carriage, and Tier Positioning in Cable Television: An Empirical Study', Review of Industrial Organization $30(3), 227-251$.

Chen, Yongmin. (2001). 'On vertical mergers and their competitive effects', RAND Journal of Economics 32(4), 667-685.

Chipty, Tasneem. (2001). 'Vertical Integration, Market Foreclosure, and Consumer Welfare in the Cable Television Industry', American Economic Review 91(3), 428-453.

Collard-Wexler, Allan, Gowrisankaran, Gautam and Lee, Robin S. (2015), "Nash-inNash" Bargaining: A Microfoundation for Applied Work. NBER Working Paper 20641.

Conlon, Christopher T. and Mortimer, Julie H. (2015), All Units Discount: Experimental Evidence from the Vending Industry. Unpublished.

Crawford, Gregory S. and Yurukoglu, Ali. (2012). 'The Welfare Effects of Bundling in Multichannel Television Markets', American Economic Review 102(2), 643-685. 
Draganska, Michaela, Klapper, Daniel and Villas-Boas, Sofia B. (2010). 'A Larger Slice or a Larger Pie? An Empirical Investigation of Bargaining Power in the Distribution Channel', Marketing Science 29(1), 57-74.

Dubin, Jeffrey A. and McFadden, Daniel L. (1984). 'An Econometric Analysis of Residential Electric Appliance Holdings and Consumption', Econometrica 52(2), 345-362.

FCC. (2012), Report and Order in MB Docket Nos. 12-68, 07-18, 05-192; Further Notice of Proposed Rulemaking in MB Docket No. 12-68; Order on Reconsideration in MB Docket No. 07-29, Technical report. FCC 12-123, released October 5, 2012.

FCC. (2013), Fifteenth Report: Annual Assessment of the Status of Competition in the Market for the Delivery of Video Programming, Technical report. FCC 13-99, released July 22, 2013.

Goolsbee, Austan and Petrin, Amil. (2004). 'Consumer Gains from Direct Broadcast Satellites and the Competition with Cable TV', Econometrica 72(2), 351-81.

Gouriéroux, Christian and Monfort, Alain. (1996), Simulation-Based Econometric Methods, Oxford University Press, Oxford.

Gowrisankaran, Gautam, Nevo, Aviv and Town, Robert. (2015). 'Mergers When Prices Are Negotiated: Evidence from the Hospital Industry', American Economic Review 105(1), 172-203.

Grennan, Matthew. (2013). 'Price Discrimination and Bargaining: Empirical Evidence from Medical Devices', American Economic Review 103(1), 147-177.

Grossman, S. and Hart, O. (1986). 'The Costs and Benefits of Ownership: A Theory of Vertical and Lateral Integration', Journal of Political Economy 94, 691-796.

Hanemann, W. Michael. (1984). 'Discrete/Continuous Models of Consumer Demand', Econometrica 52(3), 541-561.

Hart, Oliver. (1995), Firms, Contracts, and Financial Structure, Oxford University Press, New York.

Hart, Oliver and Tirole, Jean. (1990). 'Vertical Integration and Market Foreclosure', Brookings Papers on Economic Activity. Microeconomics 1990, 205-286.

Hastings, Justine S. (2004). 'Vertical Relationships and Competition in Retail Gasoline Markets: Empirical Evidence from Contract Changes in Southern California', American Economic Review $94,317-328$.

Hastings, Justine S. and Gilbert, Richard J. (2005). 'Market Power, Vertical Integration and the Wholesale Price of Gasoline', Journal of Industrial Economics 53(4), 469-492.

Ho, Kate and Lee, Robin S. (2017). 'Insurer Competition in Health Care Markets', Econometrica 85(2), 379-417. 
Horn, Hendrick and Wolinsky, Asher. (1988). 'Bilateral Monopoly and Incentives for Merger', The RAND Journal of Economics 19, 408-419.

Hortacsu, Ali and Syverson, Chad. (2007). 'Cementing Relationships: Vertical Integration, Foreclosure, Productivity, and Prices', Journal of Political Economy 115(2), 250-301.

Houde, Jean-Francois. (2012). 'Spatial Differentiation and Vertical Mergers in Retail Markets for Gasoline', American Economic Review 102(5), 2147-2182.

Krattenmaker, Thomas G. and Salop, Steven C. (1986). 'Anticompetitive Exclusion: Raising Rivals' Costs to Achieve Power over Price', The Yale Law Journal 2, 209-293.

Lee, Robin S. (2013). 'Vertical Integration and Exclusivity in Platform and Two-Sided Markets', American Economic Review 103(7), 2960-3000.

Michel, Christian. (2013), Identification and Estimation of Intra-Firm and Industry Competition via Ownership Change. Unpublished.

Mortimer, Julie H. (2008). 'Vertical Contracts in the Video Rental Industry', Review of Economic Studies 75, 165-199.

Nocke, Volker and White, Lucy. (2007). 'Do Vertical Mergers Facilitate Upstream Collusion', American Economic Review 97(4), 1321-1339.

O'Brien, Daniel P. and Salop, Steven C. (2000). 'Competitive Effects of Partial Ownership: Financial Interest and Corporate Control', Antitrust Law Journal 67, 559-614.

Ordover, J., Saloner, G. and Salop, S. (1990). 'Equilibrium Vertical Foreclosure', American Economic Review 80, 127-142.

Perry, Martin K. (1990), Vertical Integration: Determinants and Effects, in Richard Schmalensee and Robert Willig., eds, 'Handbook of Industrial Organization', Vol. 1, North Holland, Amsterdam.

Rey, Patrick and Tirole, Jean. (2007), A Primer on Foreclosure, in Mark Armstrong and Rob Porter., eds, 'Handbook of Industrial Organization', Vol. 3, North-Holland Press, Amsterdam.

Riordan, Michael H. (2008), Competitive Effects of Vertical Integration, in Paolo Buccirossi., ed., 'Handbook of Antitrust Economics', MIT Press, Cambridge, MA.

Rogerson, William P. (2014), Vertical Mergers in the Video Programming and Distribution Industry: The Case of Comcast-NBCU, in Jr. John E. Kwoka and Lawrence J. White., eds, 'The Antitrust Revolution, 6th Edition', Oxford University Press, New York.

Salop, Steven C. and Scheffman, David T. (1983). 'Raising Rivals' Costs', American Economic Review 73(2), 267-271. 
Shepard, Andrea. (1993). 'Contractual Form, Retail Price, and Asset Characteristics in Gasoline Retailing', RAND Journal of Economics 24(1), 58-77.

Spengler, Joseph J. (1950). 'Vertical integration and antitrust policy', The Journal of Political Economy pp. 347-352.

Suzuki, Ayako. (2009). 'Market foreclosure and vertical merger: A case study of the vertical merger between Turner Broadcasting and Time Warner', International Journal of Industrial Organization 27(4), 523-543.

Villas-Boas, Sofia B. (2007). 'Vertical Relationships between Manufacturers and Retailers: Inference with Limited Data', Review of Economic Studies 74(2), 625-652.

Waterman, David and Weiss, Andrew A. (1996). 'The Effects of Vertical Integration between Cable Television Systems and Pay Cable Networks', Journal of Econometrics 72(1-2), 357-95.

Willamson, Oliver. (1985), The Economic Institutions of Capitalism, New York, NY. 


\section{A Appendix Tables}

Table A.1: Sample Statistics - Prices, Market Shares, and Channels

\begin{tabular}{|c|c|c|c|c|c|c|c|c|c|}
\hline & \multirow[b]{2}{*}{ \# Obs } & \multicolumn{4}{|c|}{ Unweighted } & \multicolumn{4}{|c|}{ Weighted by HHs } \\
\hline & & Mean & StdDev & Min & $\operatorname{Max}$ & Mean & StdDev & Min & Max \\
\hline Total Markets & 6,928 & 6,928 & & & & & & & \\
\hline Average Households (millions) & 6,928 & & & & & 39.7 & & & \\
\hline \multicolumn{10}{|l|}{ Cable } \\
\hline Year & 6,928 & 2004 & 2.9 & 2000 & 2010 & 2004 & 2.8 & 2000 & 2010 \\
\hline Price & 6,928 & $\$ 50.67$ & $\$ 10.31$ & $\$ 8.67$ & $\$ 130.96$ & $\$ 52.20$ & $\$ 8.86$ & $\$ 8.67$ & $\$ 130.96$ \\
\hline Market Share & 6,928 & 0.628 & 0.162 & 0.001 & 0.965 & 0.639 & 0.135 & 0.001 & 0.965 \\
\hline Cable Networks & 6,928 & 67.0 & 18.1 & 0 & 101 & 72.1 & 14.8 & 0 & 101 \\
\hline RSNs & 6,928 & 1.6 & 0.8 & 0 & 5 & 1.8 & 0.9 & 0 & 5 \\
\hline Total Channels & 6,928 & 68.6 & 18.4 & 0 & 103 & 73.8 & 15.0 & 0 & 103 \\
\hline \multicolumn{10}{|l|}{ DirecTV } \\
\hline Year & 6,928 & 2004 & 2.9 & 2000 & 2010 & 2004 & 2.8 & 2000 & 2010 \\
\hline Price & 6,928 & $\$ 52.76$ & $\$ 6.36$ & $\$ 46.05$ & $\$ 76.73$ & $\$ 52.66$ & $\$ 6.08$ & $\$ 46.05$ & $\$ 76.73$ \\
\hline Market Share & 6,928 & 0.090 & 0.060 & 0.002 & 0.499 & 0.091 & 0.063 & 0.002 & 0.499 \\
\hline Cable Networks & 6,928 & 79.4 & 10.4 & 66 & 97 & 79.8 & 10.2 & 66 & 97 \\
\hline RSNs & 6,928 & 1.9 & 1.0 & 0 & 7 & 1.9 & 1.0 & 0 & 7 \\
\hline Total Channels & 6,928 & 84.3 & 10.9 & 69 & 107 & 84.7 & 10.7 & 69 & 107 \\
\hline \multicolumn{10}{|l|}{ Dish } \\
\hline Year & 6,928 & 2004 & 2.9 & 2000 & 2010 & 2004 & 2.8 & 2000 & 2010 \\
\hline Price & 6,928 & $\$ 53.43$ & $\$ 4.81$ & $\$ 44.28$ & $\$ 68.33$ & $\$ 53.47$ & $\$ 4.63$ & $\$ 44.28$ & $\$ 68.33$ \\
\hline Market Share & 6,928 & 0.062 & 0.054 & 0.000 & 0.472 & 0.058 & 0.052 & 0.000 & 0.472 \\
\hline Cable Networks & 6,928 & 69.4 & 13.3 & 54 & 91 & 70.0 & 13.1 & 54 & 91 \\
\hline RSNs & 6,928 & 1.8 & 0.9 & 0 & 6 & 1.7 & 0.8 & 0 & 6 \\
\hline Total Channels & 6,928 & 73.7 & 13.9 & 56 & 99 & 74.4 & 13.6 & 56 & 99 \\
\hline
\end{tabular}

Notes: Reported are the average price, market share, and number of cable networks, Regional Sport Networks (RSNs), and total channels for the local cable operators as well as the two national satellite providers serving each of our markets. Markets are defined as the set of continuous zip codes within a cable system facing the same portfolio of competitors. We exclude (the relatively few) markets facing competition between cable operators. All the data cover the years 2000-2010. To be included, we required information on each of price, market share, and channels. Cable system subscriber and channel information is from the Nielsen FOCUS dataset. Cable system price information is drawn from the Internet Archive, newspaper reports, and the TNS Bill Harvesting database. Satellite system channel and price information is drawn from the Internet Archive. Cable and satellite subscriber market shares are estimated from the MRI (2000-2007) and Simmons (2008-2010) household surveys and Nielsen FOCUS dataset. See the text for more details. 
Table A.2: National Cable Channels: Affiliate Fees and Viewership

\begin{tabular}{|c|c|c|c|c|c|c|c|c|c|c|c|}
\hline & \multicolumn{5}{|c|}{ "Affiliate Fees } & \multicolumn{6}{|c|}{ Viewership } \\
\hline & \multicolumn{5}{|c|}{ Kagan } & \multicolumn{2}{|c|}{ Nielsen Ratings } & \multicolumn{4}{|c|}{ Combined MRI / Simmons } \\
\hline & Years & Mean & StDev & Min & $\operatorname{Max}$ & Obs & Mean & Obs & Mean & SDev & Frac $>0$ \\
\hline ABC Family Channel & 11 & $\$ 0.19$ & $\$ 0.02$ & $\$ 0.16$ & $\$ 0.22$ & 747 & 0.418 & 277,535 & 0.344 & 1.149 & 0.176 \\
\hline $\mathrm{AMC}$ & 11 & $\$ 0.22$ & $\$ 0.02$ & $\$ 0.20$ & $\$ 0.25$ & 747 & 0.491 & 277,535 & 0.351 & 1.183 & 0.156 \\
\hline Animal Planet & 11 & $\$ 0.07$ & $\$ 0.01$ & $\$ 0.06$ & $\$ 0.09$ & 747 & 0.275 & 277,535 & 0.344 & 1.108 & 0.203 \\
\hline $\mathrm{A} \& \mathrm{E}$ & 11 & $\$ 0.21$ & $\$ 0.03$ & $\$ 0.16$ & $\$ 0.26$ & 747 & 0.664 & 277,535 & 0.472 & 1.373 & 0.230 \\
\hline BET & 11 & $\$ 0.14$ & $\$ 0.02$ & $\$ 0.11$ & $\$ 0.17$ & 747 & 0.382 & 277,535 & 0.184 & 1.017 & 0.070 \\
\hline Bravo & 11 & $\$ 0.15$ & $\$ 0.03$ & $\$ 0.11$ & $\$ 0.20$ & 747 & 0.277 & 277,535 & 0.169 & 0.804 & 0.092 \\
\hline Cartoon Network & 11 & $\$ 0.14$ & $\$ 0.03$ & $\$ 0.08$ & $\$ 0.18$ & 747 & 0.989 & 277,535 & 0.231 & 1.098 & 0.106 \\
\hline CMT & 11 & $\$ 0.06$ & $\$ 0.02$ & $\$ 0.01$ & $\$ 0.08$ & 747 & 0.142 & 277,535 & 0.120 & 0.732 & 0.067 \\
\hline CNBC & 11 & $\$ 0.24$ & $\$ 0.04$ & $\$ 0.16$ & $\$ 0.30$ & 747 & 0.217 & 277,535 & 0.313 & 1.185 & 0.170 \\
\hline $\mathrm{CNN}$ & 11 & $\$ 0.43$ & $\$ 0.05$ & $\$ 0.35$ & $\$ 0.52$ & 747 & 0.550 & 277,535 & 0.701 & 1.744 & 0.319 \\
\hline Comedy Central & 11 & $\$ 0.11$ & $\$ 0.02$ & $\$ 0.08$ & $\$ 0.14$ & 747 & 0.449 & 277,535 & 0.280 & 0.997 & 0.162 \\
\hline Discovery Channel & 11 & $\$ 0.27$ & $\$ 0.04$ & $\$ 0.22$ & $\$ 0.35$ & 747 & 0.535 & 277,535 & 0.628 & 1.462 & 0.327 \\
\hline Disney Channel & 11 & $\$ 0.81$ & $\$ 0.06$ & $\$ 0.75$ & $\$ 0.91$ & 747 & 1.171 & 277,535 & 0.246 & 1.074 & 0.116 \\
\hline E! Entertainment TV & 11 & $\$ 0.19$ & $\$ 0.02$ & $\$ 0.15$ & $\$ 0.21$ & 747 & 0.315 & 277,535 & 0.201 & 0.788 & 0.137 \\
\hline ESPN & 11 & $\$ 2.81$ & $\$ 1.12$ & $\$ 1.14$ & $\$ 4.34$ & 747 & 0.836 & 277,535 & 0.675 & 1.767 & 0.257 \\
\hline ESPN 2 & 11 & $\$ 0.37$ & $\$ 0.14$ & $\$ 0.17$ & $\$ 0.58$ & 747 & 0.262 & 277,535 & 0.334 & 1.220 & 0.151 \\
\hline ESPN Classic Sports & 11 & $\$ 0.14$ & $\$ 0.03$ & $\$ 0.10$ & $\$ 0.18$ & 636 & 0.037 & 277,535 & 0.072 & 0.521 & 0.047 \\
\hline Food Network & 11 & $\$ 0.06$ & $\$ 0.03$ & $\$ 0.03$ & $\$ 0.14$ & 747 & 0.411 & 277,535 & 0.396 & 1.364 & 0.175 \\
\hline Fox News Channel & 11 & $\$ 0.32$ & $\$ 0.18$ & $\$ 0.17$ & $\$ 0.70$ & 747 & 0.785 & 277,535 & 0.697 & 1.961 & 0.267 \\
\hline $\mathrm{FX}$ & 11 & $\$ 0.34$ & $\$ 0.06$ & $\$ 0.27$ & $\$ 0.43$ & 747 & 0.463 & 277,535 & 0.258 & 0.976 & 0.137 \\
\hline Golf Channel & 11 & $\$ 0.20$ & $\$ 0.05$ & $\$ 0.13$ & $\$ 0.26$ & 580 & 0.065 & 277,535 & 0.084 & 0.633 & 0.041 \\
\hline Hallmark Channel & 11 & $\$ 0.04$ & $\$ 0.02$ & $\$ 0.01$ & $\$ 0.06$ & 699 & 0.307 & 225,618 & 0.301 & 1.268 & 0.088 \\
\hline Headline News & - & - & - & - & - & 747 & 0.214 & 277,535 & 0.278 & 0.983 & 0.173 \\
\hline HGTV & 11 & $\$ 0.08$ & $\$ 0.04$ & $\$ 0.03$ & $\$ 0.14$ & 747 & 0.500 & 277,535 & 0.397 & 1.446 & 0.162 \\
\hline History Channel & 11 & $\$ 0.18$ & $\$ 0.04$ & $\$ 0.13$ & $\$ 0.23$ & 747 & 0.531 & 277,535 & 0.531 & 1.462 & 0.251 \\
\hline Lifetime & 11 & $\$ 0.21$ & $\$ 0.06$ & $\$ 0.13$ & $\$ 0.29$ & 747 & 0.679 & 277,535 & 0.554 & 1.650 & 0.199 \\
\hline MSNBC & 11 & $\$ 0.14$ & $\$ 0.02$ & $\$ 0.12$ & $\$ 0.17$ & 747 & 0.343 & 277,535 & 0.330 & 1.181 & 0.182 \\
\hline MTV & 11 & $\$ 0.27$ & $\$ 0.05$ & $\$ 0.20$ & $\$ 0.35$ & 747 & 0.568 & 277,535 & 0.235 & 0.983 & 0.127 \\
\hline Nickelodeon & 11 & $\$ 0.37$ & $\$ 0.05$ & $\$ 0.29$ & $\$ 0.47$ & 747 & 1.555 & 277,535 & 0.200 & 0.991 & 0.096 \\
\hline SyFy & 11 & $\$ 0.17$ & $\$ 0.04$ & $\$ 0.12$ & $\$ 0.22$ & 747 & 0.427 & 277,535 & 0.301 & 1.207 & 0.126 \\
\hline TBS & 11 & $\$ 0.37$ & $\$ 0.12$ & $\$ 0.19$ & $\$ 0.54$ & 747 & 0.905 & 277,535 & 0.497 & 1.345 & 0.243 \\
\hline TLC & 11 & $\$ 0.16$ & $\$ 0.01$ & $\$ 0.14$ & $\$ 0.17$ & 747 & 0.422 & 277,535 & 0.342 & 1.151 & 0.173 \\
\hline truTV & 11 & $\$ 0.09$ & $\$ 0.01$ & $\$ 0.08$ & $\$ 0.10$ & 747 & 0.384 & 277,535 & 0.233 & 1.081 & 0.101 \\
\hline Turner Classic Movies & 11 & $\$ 0.22$ & $\$ 0.03$ & $\$ 0.16$ & $\$ 0.27$ & 580 & 0.286 & 277,535 & 0.268 & 1.142 & 0.105 \\
\hline TNT & 11 & $\$ 0.83$ & $\$ 0.16$ & $\$ 0.55$ & $\$ 1.10$ & 747 & 1.219 & 277,535 & 0.592 & 1.553 & 0.263 \\
\hline USA & 11 & $\$ 0.46$ & $\$ 0.07$ & $\$ 0.36$ & $\$ 0.57$ & 747 & 1.081 & 277,535 & 0.503 & 1.442 & 0.230 \\
\hline VH1 & 11 & $\$ 0.12$ & $\$ 0.02$ & $\$ 0.09$ & $\$ 0.16$ & 747 & 0.336 & 277,535 & 0.151 & 0.717 & 0.101 \\
\hline Weather Channel & 11 & $\$ 0.10$ & $\$ 0.01$ & $\$ 0.08$ & $\$ 0.12$ & 747 & 0.234 & 277,535 & 0.380 & 1.046 & 0.326 \\
\hline
\end{tabular}

Notes: Reported are average affiliate fees and viewership of the 38 non-RSN cable television networks included in our demand system. The averages are over the years 2000-2010 for SNL Kagan affiliate fees, over DMA-years for the Nielsen (DMA-level) viewership data, and over households and years for the MRI (2000-2007) and Simmons (2008-2010) household-level viewership data. Affiliate fees are the monthly per subscriber fees paid by cable and satellite distributors to television networks for the right to distribute the network's programming to subscribers. The Nielsen "rating" is the percentage of US households watching a given program on a given channel at a given time. We average program-level ratings across programs within a channel-DMA-year, and report the across-DMA-year average here. MRI/Simmons viewership is reported as the average number of hours watching that channel in a typical week. It is converted to a Nielsen-equivalent "rating" by dividing by the number of hours in a week and rescaling it to lie between 0 and 100. The average fraction of households viewing a channel at all ("Frac $>0$ ") is the average percentage of households that reported positive viewing of a channel in a typical week. 


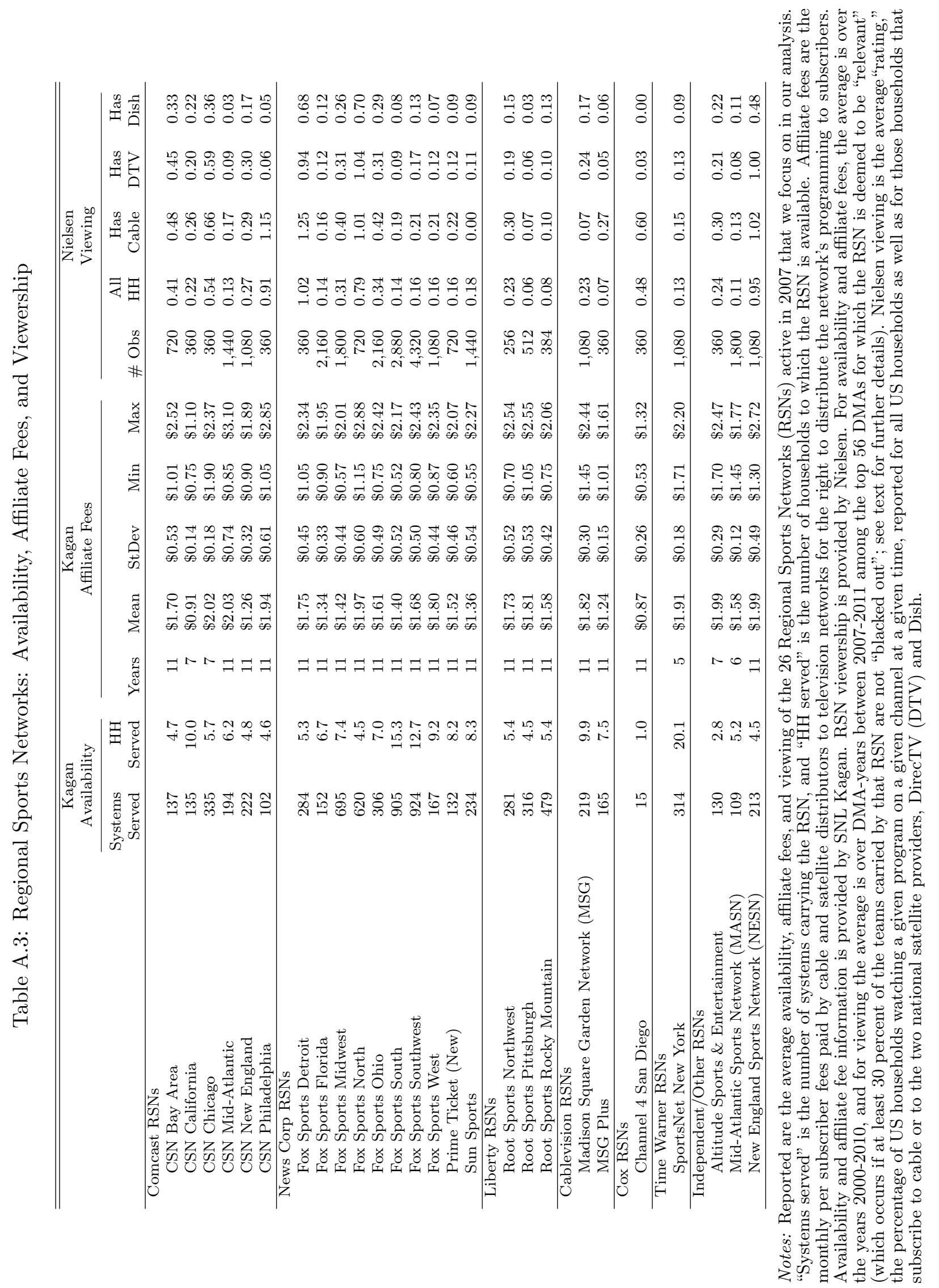




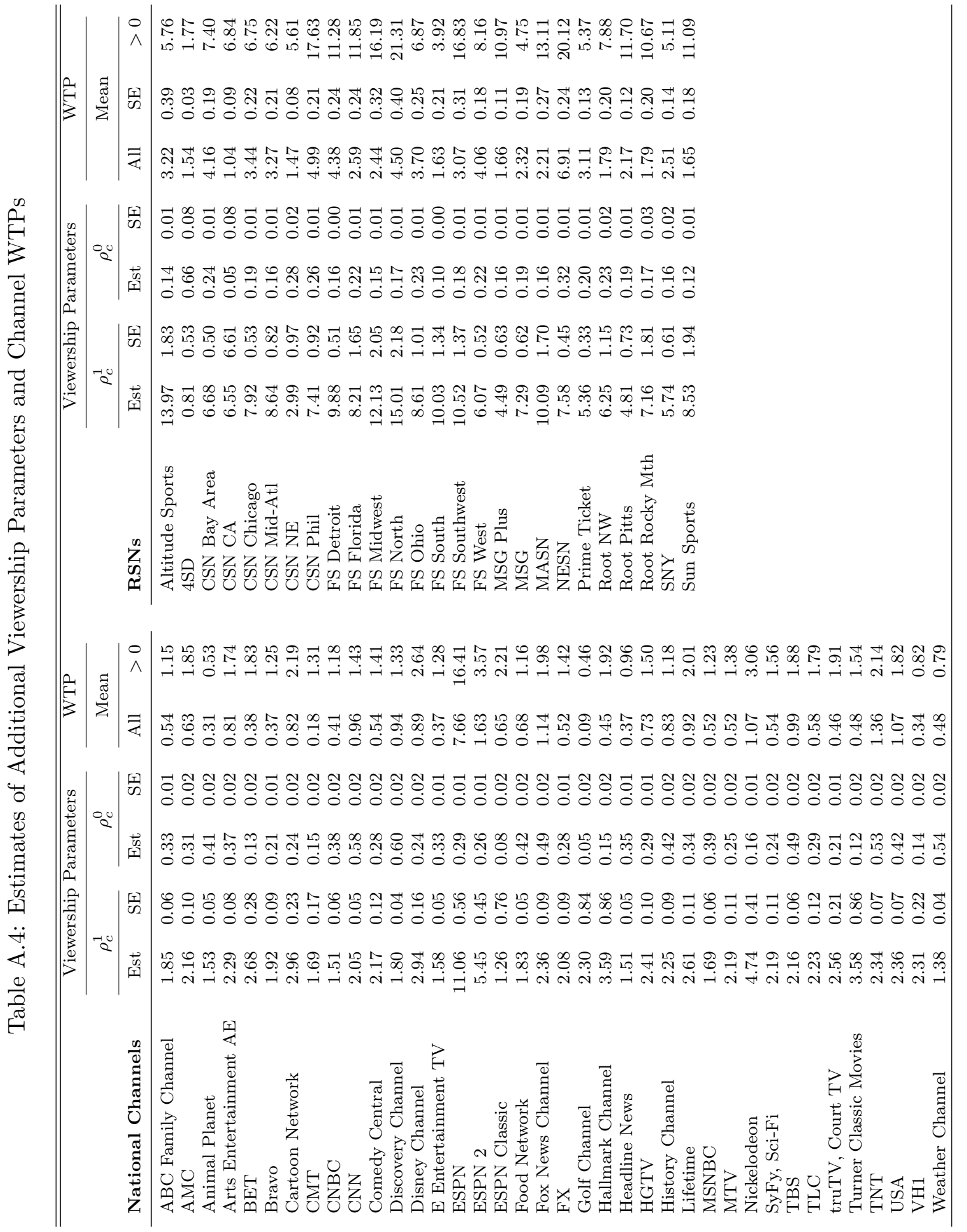

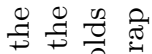
응 $\therefore 2$ दิ

능

घี ซ్రై

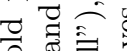

论

可

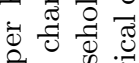

o

๘ี

궁

$\Xi$ 恶串

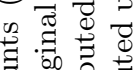

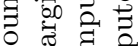

द् घี ठे

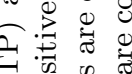

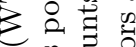

路 윰

ซึ च

잉

w

다웡

园䨔

5 .

풀

0.

영

긍

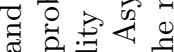

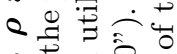

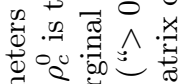

要

䂧

द्व

霑

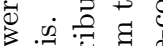

$5 \geq$.

ช

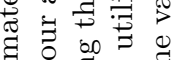

g.

के

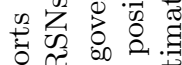

论

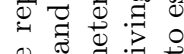

品

๙

胥

즈 ฮี 융

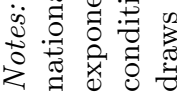


Table A.5: Simulated Market Outcomes for RSNs (1/6)

\begin{tabular}{|c|c|c|c|c|c|c|}
\hline & & \multirow[t]{2}{*}{ (i) No VI } & \multicolumn{2}{|c|}{ (ii) VI PARs } & \multicolumn{2}{|c|}{ (iii) VI No PARs } \\
\hline & & & \multicolumn{2}{|c|}{ (vs. No VI) } & \multicolumn{2}{|c|}{ (vs. No VI) } \\
\hline & & Level & $\% \Delta_{l v l}$ & $\% \Delta_{W T P}$ & $\% \Delta_{l v l}$ & $\% \Delta_{W T P}$ \\
\hline \multicolumn{7}{|c|}{ CABLE OWNED RSNs (1/2) } \\
\hline $4 \mathrm{SD}$ & Cable Mkt Share & 0.74 & $0.1 \%$ & & $1.4 \%$ & \\
\hline Cox & & {$[0.66,0.77]$} & {$[-0.7 \%, 3.3 \%]$} & & {$[-0.1 \%, 7.9 \%]$} & \\
\hline Pop $2.81 \mathrm{M}$ & Sat Mkt Share & 0.16 & $0.0 \%$ & & $-5.6 \%$ & \\
\hline Footprint $100 \%$ & & {$[0.15,0.19]$} & {$[-3.6 \%, 2.6 \%]$} & & {$[-13.4 \%, 0.5 \%]$} & \\
\hline \multirow[t]{4}{*}{ WTP $\$ 1.54$} & Cable Carriage & 0.49 & $0.0 \%$ & & $0.0 \%$ & \\
\hline & & {$[0.49,0.49]$} & {$[0.0 \%, 105.5 \%]$} & & {$[0.0 \%, 105.5 \%]$} & \\
\hline & Cable Prices & 51.05 & $0.0 \%$ & & $-0.2 \%$ & \\
\hline & & {$[50.00,55.39]$} & {$[-2.3 \%, 0.4 \%]$} & & {$[-4.5 \%, 0.6 \%]$} & \\
\hline \multirow[t]{10}{*}{ Foreclose: $69 \%$} & Aff Fees to Sat & 0.78 & $1.2 \%$ & & - & \\
\hline & & {$[-0.07,1.45]$} & {$[-13.4 \%, 18.9 \%]$} & & & \\
\hline & Cable + RSN Surplus & 28.87 & $0.0 \%$ & $0.1 \%$ & $0.3 \%$ & $5.9 \%$ \\
\hline & & {$[16.84,34.38]$} & {$[0.0 \%, 2.2 \%]$} & {$[0.0 \%, 87.9 \%]$} & {$[0.0 \%, 3.1 \%]$} & {$[1.3 \%, 93.5 \%]$} \\
\hline & Satellite Surplus & 3.71 & $-0.1 \%$ & $-0.2 \%$ & $-2.3 \%$ & $-5.4 \%$ \\
\hline & & {$[1.08,4.37]$} & {$[-3.9 \%, 2.5 \%]$} & {$[-12.5 \%, 14.6 \%]$} & {$[-11.2 \%, 0.0 \%]$} & {$[-27.9 \%, 0.0 \%]$} \\
\hline & Consumer Welfare & 34.43 & $0.0 \%$ & $0.7 \%$ & $-0.4 \%$ & $-8.3 \%$ \\
\hline & & {$[18.90,38.61]$} & {$[-0.3 \%, 2.1 \%]$} & {$[-18.9 \%, 72.8 \%]$} & {$[-1.8 \%, 5.0 \%]$} & {$[-32.8 \%, 112.3 \%]$} \\
\hline & Total Welfare & 67.01 & $0.0 \%$ & $0.6 \%$ & $-0.2 \%$ & $-7.8 \%$ \\
\hline & & {$[34.21,73.77]$} & {$[-0.1 \%, 1.6 \%]$} & {$[-5.2 \%, 148.2 \%]$} & {$[-0.7 \%, 2.3 \%]$} & {$[-27.1 \%, 165.7 \%]$} \\
\hline CSN BAY AREA & Cable Mkt Share & 0.61 & $2.3 \%$ & & $2.2 \%$ & \\
\hline Comcast & & {$[0.57,0.65]$} & {$[0.8 \%, 7.5 \%]$} & & {$[0.8 \%, 7.5 \%]$} & \\
\hline Pop $6.03 \mathrm{M}$ & Sat Mkt Share & 0.22 & $-1.9 \%$ & & $-1.8 \%$ & \\
\hline Footprint $54 \%$ & New & {$[0.21,0.24]$} & {$[-7.3 \%,-0.8 \%]$} & & {$[-7.3 \%,-0.7 \%]$} & \\
\hline WTP $\$ 4.16$ & Cable Carriage & 0.57 & $0.0 \%$ & & $0.0 \%$ & \\
\hline & & {$[0.48,0.62]$} & {$[-8.1 \%, 9.8 \%]$} & & {$[-8.1 \%, 9.8 \%]$} & \\
\hline & Cable Prices & 55.64 & $-1.7 \%$ & & $-1.7 \%$ & \\
\hline & & {$[53.77,58.92]$} & {$[-4.6 \%,-0.2 \%]$} & & {$[-4.6 \%,-0.2 \%]$} & \\
\hline Foreclose: $0 \%$ & Aff Fees to Sat & 1.69 & $0.5 \%$ & & $17.7 \%$ & \\
\hline & & {$[0.76,2.01]$} & {$[-3.8 \%, 9.3 \%]$} & & {$[8.6 \%, 32.7 \%]$} & \\
\hline & Cable + RSN Surplus & 23.96 & $0.1 \%$ & $0.4 \%$ & $0.3 \%$ & $1.9 \%$ \\
\hline & & {$[13.04,26.89]$} & {$[-0.4 \%, 0.5 \%]$} & {$[-3.6 \%, 2.8 \%]$} & {$[-0.2 \%, 0.8 \%]$} & {$[-1.6 \%, 4.4 \%]$} \\
\hline & Satellite Surplus & 5.62 & $-2.0 \%$ & $-2.7 \%$ & $-3.1 \%$ & $-4.2 \%$ \\
\hline & & {$[1.69,6.16]$} & {$[-8.2 \%,-0.9 \%]$} & {$[-10.8 \%,-1.5 \%]$} & {$[-9.5 \%,-1.6 \%]$} & {$[-12.8 \%,-2.9 \%]$} \\
\hline & Consumer Welfare & 33.25 & $1.3 \%$ & $10.6 \%$ & $1.3 \%$ & $10.5 \%$ \\
\hline & & {$[17.78,37.30]$} & {$[0.5 \%, 4.4 \%]$} & {$[4.4 \%, 37.6 \%]$} & {$[0.5 \%, 4.4 \%]$} & {$[4.1 \%, 37.5 \%]$} \\
\hline & Total Welfare & 62.83 & $0.5 \%$ & $8.3 \%$ & $0.5 \%$ & $8.2 \%$ \\
\hline & & {$[29.93,68.46]$} & {$[0.0 \%, 1.8 \%]$} & {$[1.3 \%, 30.6 \%]$} & {$[0.0 \%, 1.8 \%]$} & {$[1.2 \%, 30.6 \%]$} \\
\hline CSN CA & Cable Mkt Share & 0.61 & $0.4 \%$ & & $0.4 \%$ & \\
\hline Comcast & & {$[0.55,0.65]$} & {$[-4.8 \%, 4.8 \%]$} & & {$[-4.8 \%, 4.8 \%]$} & \\
\hline Pop $3.86 \mathrm{M}$ & Sat Mkt Share & 0.26 & $-0.2 \%$ & & $-0.2 \%$ & \\
\hline Footprint $10 \%$ & & {$[0.23,0.29]$} & {$[-3.5 \%, 6.4 \%]$} & & {$[-3.5 \%, 6.4 \%]$} & \\
\hline WTP \$1.04 & Cable Carriage & 0.36 & $0.0 \%$ & & $0.0 \%$ & \\
\hline & & {$[0.32,0.36]$} & {$[0.0 \%, 64.4 \%]$} & & {$[0.0 \%, 64.4 \%]$} & \\
\hline & Cable Prices & 53.88 & $-0.2 \%$ & & $-0.2 \%$ & \\
\hline & & {$[51.89,57.25]$} & {$[-3.0 \%, 3.3 \%]$} & & {$[-3.0 \%, 3.4 \%]$} & \\
\hline Foreclose: $0 \%$ & Aff Fees to Sat & 0.14 & $0.2 \%$ & & $5.9 \%$ & \\
\hline & & {$[-0.08,0.61]$} & {$[-26.0 \%, 15.1 \%]$} & & {$[-8.4 \%, 45.7 \%]$} & \\
\hline & Cable + RSN Surplus & 23.13 & $0.0 \%$ & $-0.1 \%$ & $0.0 \%$ & $0.1 \%$ \\
\hline & & {$[12.36,26.22]$} & {$[0.0 \%, 0.4 \%]$} & {$[-0.2 \%, 14.8 \%]$} & {$[0.0 \%, 0.5 \%]$} & {$[0.0 \%, 16.9 \%]$} \\
\hline & Satellite Surplus & 6.32 & $-0.2 \%$ & $-1.1 \%$ & $-0.2 \%$ & $-1.3 \%$ \\
\hline & & {$[1.92,6.81]$} & {$[-3.6 \%, 6.7 \%]$} & {$[-44.3 \%, 24.5 \%]$} & {$[-3.8 \%, 6.6 \%]$} & {$[-46.3 \%, 24.1 \%]$} \\
\hline & Consumer Welfare & 35.17 & $0.2 \%$ & $6.6 \%$ & $0.2 \%$ & $6.6 \%$ \\
\hline & & {$[18.63,39.77]$} & {$[-2.2 \%, 2.2 \%]$} & {$[-54.6 \%, 135.7 \%]$} & {$[-2.2 \%, 2.2 \%]$} & {$[-54.7 \%, 135.7 \%]$} \\
\hline & Total Welfare & 64.62 & $0.1 \%$ & $5.4 \%$ & $0.1 \%$ & $5.4 \%$ \\
\hline & & {$[30.68,71.11]$} & {$[-0.6 \%, 1.0 \%]$} & {$[-26.1 \%, 109.5 \%]$} & {$[-0.6 \%, 1.0 \%]$} & {$[-26.1 \%, 109.4 \%]$} \\
\hline CSN CHICAGO & Cable Mkt Share & 0.58 & $1.2 \%$ & & $0.8 \%$ & \\
\hline Comcast & & {$[0.57,0.62]$} & {$[-0.1 \%, 1.5 \%]$} & & {$[-0.1 \%, 1.4 \%]$} & \\
\hline Pop $9.62 \mathrm{M}$ & Sat Mkt Share & 0.23 & $-0.8 \%$ & & $-0.6 \%$ & \\
\hline Footprint $76 \%$ & & {$[0.22,0.23]$} & {$[-1.5 \%, 0.2 \%]$} & & {$[-1.4 \%, 0.2 \%]$} & \\
\hline WTP $\$ 3.44$ & Cable Carriage & 0.78 & $-1.3 \%$ & & $-1.3 \%$ & \\
\hline & & {$[0.70,0.79]$} & {$[-0.4 \%, 19.4 \%]$} & & {$[-0.5 \%, 18.8 \%]$} & \\
\hline & Cable Prices & 58.98 & $-0.7 \%$ & & $-0.4 \%$ & \\
\hline & & {$[56.78,59.57]$} & {$[-0.8 \%, 0.3 \%]$} & & {$[-0.8 \%, 0.3 \%]$} & \\
\hline Foreclose: $0 \%$ & Aff Fees to Sat & 1.59 & $0.2 \%$ & & $12.5 \%$ & \\
\hline & & {$[0.65,1.75]$} & {$[-6.1 \%, 1.1 \%]$} & & {$[4.4 \%, 19.4 \%]$} & \\
\hline & Cable + RSN Surplus & 22.55 & $0.0 \%$ & $0.0 \%$ & $0.2 \%$ & $1.2 \%$ \\
\hline & & {$[10.47,25.54]$} & {$[0.0 \%, 0.8 \%]$} & {$[-0.2 \%, 5.5 \%]$} & {$[0.1 \%, 1.0 \%]$} & {$[0.9 \%, 6.6 \%]$} \\
\hline & Satellite Surplus & 5.78 & $-0.8 \%$ & $-1.4 \%$ & $-1.3 \%$ & $-2.2 \%$ \\
\hline & & {$[1.81,6.21]$} & {$[-1.4 \%, 0.2 \%]$} & {$[-2.0 \%, 0.3 \%]$} & {$[-2.4 \%,-0.4 \%]$} & {$[-3.1 \%,-0.9 \%]$} \\
\hline & Consumer Welfare & 31.69 & $0.6 \%$ & $5.9 \%$ & $0.4 \%$ & $3.9 \%$ \\
\hline & & {$[16.88,35.79]$} & {$[-0.1 \%, 1.1 \%]$} & {$[-0.8 \%, 10.5 \%]$} & {$[-0.1 \%, 1.0 \%]$} & {$[-0.9 \%, 9.1 \%]$} \\
\hline & Total Welfare & 60.01 & $0.3 \%$ & $4.5 \%$ & $0.2 \%$ & $2.9 \%$ \\
\hline & & {$[27.90,66.79]$} & {$[0.0 \%, 0.7 \%]$} & {$[-0.7 \%, 11.8 \%]$} & {$[0.0 \%, 0.7 \%]$} & {$[-0.7 \%, 11.6 \%]$} \\
\hline
\end{tabular}

Notes: Simulated market outcomes across three integration scenarios. See Table 4 for details. 
Table A.6: Simulated Market Outcomes for RSNs (2/6)

\begin{tabular}{|c|c|c|c|c|c|c|}
\hline & & \multirow[t]{2}{*}{ (i) No VI } & \multicolumn{2}{|c|}{ (ii) VI PARs } & \multicolumn{2}{|c|}{ (iii) VI No PARs } \\
\hline & & & \multicolumn{2}{|c|}{ (vs. No VI) } & \multicolumn{2}{|c|}{ (vs. No VI) } \\
\hline & & Level & $\% \Delta_{l v l}$ & $\% \Delta_{W T P}$ & $\% \Delta_{l v l}$ & $\% \Delta_{W T P}$ \\
\hline \multicolumn{7}{|c|}{ CABLE OWNED RSNs (2/2) } \\
\hline CSN MID-ATL & Cable Mkt Share & 0.65 & $0.6 \%$ & & $0.3 \%$ & \\
\hline Comcast & & {$[0.62,0.66]$} & {$[0.1 \%, 3.4 \%]$} & & {$[-0.5 \%, 3.7 \%]$} & \\
\hline Pop $6.55 \mathrm{M}$ & Sat Mkt Share & 0.18 & $-0.4 \%$ & & $-0.1 \%$ & \\
\hline Footprint $70 \%$ & & {$[0.18,0.19]$} & {$[-2.8 \%, 0.0 \%]$} & & {$[-9.3 \%, 0.2 \%]$} & \\
\hline \multirow[t]{4}{*}{ Pred WTP $\$ 3.27$} & Cable Carriage & 0.59 & $2.9 \%$ & & $5.5 \%$ & \\
\hline & & {$[0.42,0.82]$} & {$[-17.6 \%, 67.1 \%]$} & & {$[-26.2 \%, 80.4 \%]$} & \\
\hline & Cable Prices & 55.63 & $-0.4 \%$ & & $-0.1 \%$ & \\
\hline & & {$[55.08,57.75]$} & {$[-1.7 \%, 0.3 \%]$} & & {$[-1.5 \%, 1.6 \%]$} & \\
\hline \multirow[t]{10}{*}{ Foreclose: $20 \%$} & Aff Fees to Sat & 1.35 & $3.0 \%$ & & $38.2 \%$ & \\
\hline & & {$[0.49,1.59]$} & {$[-10.3 \%, 9.0 \%]$} & & {$[13.5 \%, 53.3 \%]$} & \\
\hline & Cable + RSN Surplus & 23.95 & $0.1 \%$ & $0.8 \%$ & $0.5 \%$ & $3.9 \%$ \\
\hline & & {$[11.54,26.29]$} & {$[-0.3 \%, 1.6 \%]$} & {$[-2.7 \%, 11.0 \%]$} & {$[-0.3 \%, 2.0 \%]$} & {$[-2.5 \%, 14.0 \%]$} \\
\hline & Satellite Surplus & 4.53 & $-0.6 \%$ & $-0.8 \%$ & $-2.3 \%$ & $-3.1 \%$ \\
\hline & & {$[1.35,5.00]$} & {$[-2.6 \%, 0.1 \%]$} & {$[-4.0 \%, 0.1 \%]$} & {$[-4.5 \%,-0.1 \%]$} & {$[-6.1 \%,-0.2 \%]$} \\
\hline & Consumer Welfare & 30.76 & $0.5 \%$ & $4.5 \%$ & $0.3 \%$ & $2.5 \%$ \\
\hline & & {$[15.74,33.88]$} & {$[0.1 \%, 2.4 \%]$} & {$[1.7 \%, 22.8 \%]$} & {$[-3.1 \%, 2.0 \%]$} & {$[-26.8 \%, 20.6 \%]$} \\
\hline & Total Welfare & 59.24 & $0.2 \%$ & $4.5 \%$ & $0.2 \%$ & $3.3 \%$ \\
\hline & & {$[27.92,65.09]$} & {$[0.2 \%, 1.4 \%]$} & {$[2.8 \%, 24.1 \%]$} & {$[-1.2 \%, 1.3 \%]$} & {$[-19.3 \%, 23.8 \%]$} \\
\hline CSN NE & Cable Mkt Share & 0.63 & $1.0 \%$ & & $1.7 \%$ & \\
\hline Comcast & & {$[0.61,0.66]$} & {$[0.0 \%, 1.6 \%]$} & & {$[0.1 \%, 2.7 \%]$} & \\
\hline Pop $5.2 \mathrm{M}$ & Sat Mkt Share & 0.13 & $-1.1 \%$ & & $-5.4 \%$ & \\
\hline Footprint $85 \%$ & & {$[0.12,0.13]$} & {$[-1.8 \%, 0.0 \%]$} & & {$[-7.2 \%,-0.2 \%]$} & \\
\hline WTP $\$ 1.47$ & Cable Carriage & 0.77 & $12.2 \%$ & & $9.7 \%$ & \\
\hline & & {$[0.62,0.83]$} & {$[3.3 \%, 33.8 \%]$} & & {$[3.3 \%, 35.5 \%]$} & \\
\hline & Cable Prices & 56.17 & $-0.5 \%$ & & $-0.4 \%$ & \\
\hline & & {$[54.37,57.34]$} & {$[-1.0 \%, 0.2 \%]$} & & {$[-1.3 \%, 0.5 \%]$} & \\
\hline Foreclose: $89 \%$ & Aff Fees to Sat & 0.78 & $-4.7 \%$ & & - & \\
\hline & & {$[0.20,0.91]$} & {$[-3.8 \%, 9.6 \%]$} & & - & \\
\hline & Cable + RSN Surplus & 26.78 & $0.2 \%$ & $3.6 \%$ & $0.6 \%$ & $10.2 \%$ \\
\hline & & {$[12.90,29.35]$} & {$[0.0 \%, 0.5 \%]$} & {$[1.4 \%, 9.7 \%]$} & {$[0.2 \%, 1.1 \%]$} & {$[4.8 \%, 18.7 \%]$} \\
\hline & Satellite Surplus & 2.98 & $-1.0 \%$ & $-2.1 \%$ & $-2.4 \%$ & $-4.8 \%$ \\
\hline & & {$[0.85,3.21]$} & {$[-1.8 \%, 0.0 \%]$} & {$[-3.4 \%,-0.1 \%]$} & {$[-3.7 \%,-0.5 \%]$} & {$[-8.2 \%,-0.9 \%]$} \\
\hline & Consumer Welfare & 29.21 & $0.8 \%$ & $15.0 \%$ & $0.1 \%$ & $2.7 \%$ \\
\hline & & {$[15.79,33.11]$} & {$[0.1 \%, 1.3 \%]$} & {$[1.7 \%, 28.7 \%]$} & {$[-0.9 \%, 1.4 \%]$} & {$[-20.0 \%, 31.8 \%]$} \\
\hline & Total Welfare & 58.97 & $0.4 \%$ & $16.5 \%$ & $0.2 \%$ & $8.1 \%$ \\
\hline & & {$[28.89,64.91]$} & {$[0.1 \%, 0.7 \%]$} & {$[4.5 \%, 30.2 \%]$} & {$[-0.2 \%, 0.8 \%]$} & {$[-6.9 \%, 34.1 \%]$} \\
\hline MSG PLUS & Cable Mkt Share & 0.66 & $3.1 \%$ & & $3.1 \%$ & \\
\hline Cablevision & & {$[0.65,0.70]$} & {$[0.1 \%, 5.1 \%]$} & & {$[0.1 \%, 4.9 \%]$} & \\
\hline Pop $9.46 \mathrm{M}$ & Sat Mkt Share & 0.19 & $-4.6 \%$ & & $-4.6 \%$ & \\
\hline Footprint $49 \%$ & & {$[0.17,0.19]$} & {$[-7.5 \%,-0.4 \%]$} & & {$[-9.4 \%,-0.7 \%]$} & \\
\hline WTP $\$ 1.66$ & Cable Carriage & 0.42 & $54.6 \%$ & & $54.6 \%$ & \\
\hline & & {$[0.41,0.89]$} & {$[-0.3 \%, 85.8 \%]$} & & {$[-23.0 \%, 85.8 \%]$} & \\
\hline & Cable Prices & 59.59 & $-2.2 \%$ & & $-2.2 \%$ & \\
\hline & & {$[57.46,61.09]$} & {$[-4.0 \%, 0.0 \%]$} & & {$[-3.8 \%, 0.8 \%]$} & \\
\hline Foreclose: $6 \%$ & Aff Fees to Sat & 0.75 & $0.9 \%$ & & $31.1 \%$ & \\
\hline & & {$[0.32,0.89]$} & {$[-17.8 \%, 17.0 \%]$} & & {$[2.6 \%, 64.0 \%]$} & \\
\hline & Cable + RSN Surplus & 32.81 & $0.6 \%$ & $11.7 \%$ & $0.7 \%$ & $14.3 \%$ \\
\hline & & {$[15.57,36.92]$} & {$[-0.1 \%, 0.9 \%]$} & {$[-2.2 \%, 17.0 \%]$} & {$[-0.1 \%, 1.3 \%]$} & {$[-1.4 \%, 24.0 \%]$} \\
\hline & Satellite Surplus & 4.39 & $-4.7 \%$ & $-12.3 \%$ & $-5.6 \%$ & $-14.8 \%$ \\
\hline & & {$[1.32,4.71]$} & {$[-7.5 \%,-0.3 \%]$} & {$[-19.7 \%,-0.9 \%]$} & {$[-7.8 \%, 0.0 \%]$} & {$[-21.6 \%,-0.1 \%]$} \\
\hline & Consumer Welfare & 35.59 & $3.2 \%$ & $67.6 \%$ & $3.1 \%$ & $67.2 \%$ \\
\hline & & {$[19.34,39.95]$} & {$[0.4 \%, 4.8 \%]$} & {$[8.2 \%, 110.9 \%]$} & {$[-1.0 \%, 4.5 \%]$} & {$[-21.2 \%, 108.9 \%]$} \\
\hline & Total Welfare & 72.78 & $1.5 \%$ & $67.0 \%$ & $1.5 \%$ & $66.7 \%$ \\
\hline & & {$[33.33,80.75]$} & {$[0.2 \%, 2.1 \%]$} & {$[10.0 \%, 96.8 \%]$} & {$[-0.2 \%, 2.0 \%]$} & {$[-9.4 \%, 95.8 \%]$} \\
\hline SNY & Cable Mkt Share & 0.63 & $3.8 \%$ & & $3.8 \%$ & \\
\hline Comcast, TWC & & {$[0.62,0.67]$} & {$[0.5 \%, 6.1 \%]$} & & {$[0.5 \%, 6.1 \%]$} & \\
\hline Pop $11.7 \mathrm{M}$ & Sat Mkt Share & 0.18 & $-4.5 \%$ & & $-4.5 \%$ & \\
\hline Footprint $35 \%$ & & {$[0.17,0.18]$} & {$[-8.2 \%,-0.7 \%]$} & & {$[-8.2 \%,-0.7 \%]$} & \\
\hline WTP $\$ 2.51$ & Cable Carriage & 0.79 & $1.9 \%$ & & $1.9 \%$ & \\
\hline & & {$[0.75,0.89]$} & {$[-10.3 \%, 8.3 \%]$} & & {$[-10.3 \%, 7.7 \%]$} & \\
\hline & Cable Prices & 59.80 & $-2.9 \%$ & & $-2.9 \%$ & \\
\hline & & {$[56.97,61.32]$} & {$[-4.5 \%,-0.3 \%]$} & & {$[-4.5 \%,-0.3 \%]$} & \\
\hline Foreclose: $0 \%$ & Aff Fees to Sat & 1.40 & $-4.0 \%$ & & $-3.9 \%$ & \\
\hline & & {$[0.45,1.54]$} & {$[-6.2 \%, 6.3 \%]$} & & {$[-6.0 \%, 6.3 \%]$} & \\
\hline & Cable + RSN Surplus & 30.12 & $0.0 \%$ & $-0.6 \%$ & $0.0 \%$ & $-0.6 \%$ \\
\hline & & {$[14.34,33.64]$} & {$[-0.6 \%, 0.2 \%]$} & {$[-9.6 \%, 2.7 \%]$} & {$[-0.6 \%, 0.2 \%]$} & {$[-9.6 \%, 2.3 \%]$} \\
\hline & Satellite Surplus & 4.16 & $-4.3 \%$ & $-7.2 \%$ & $-4.3 \%$ & $-7.2 \%$ \\
\hline & & {$[1.26,4.49]$} & {$[-8.4 \%,-0.7 \%]$} & {$[-13.8 \%,-1.1 \%]$} & {$[-8.4 \%,-0.7 \%]$} & {$[-13.8 \%,-1.1 \%]$} \\
\hline & Consumer Welfare & 33.69 & $3.3 \%$ & $43.9 \%$ & $3.3 \%$ & $43.9 \%$ \\
\hline & & {$[18.22,37.96]$} & {$[0.4 \%, 5.2 \%]$} & {$[5.9 \%, 72.8 \%]$} & {$[0.4 \%, 5.2 \%]$} & {$[5.9 \%, 72.8 \%]$} \\
\hline & Total Welfare & 67.96 & $1.3 \%$ & $36.1 \%$ & $1.3 \%$ & $36.1 \%$ \\
\hline & & {$[31.47,75.26]$} & {$[0.2 \%, 2.0 \%]$} & {$[4.2 \%, 58.3 \%]$} & {$[0.2 \%, 2.0 \%]$} & {$[4.2 \%, 58.3 \%]$} \\
\hline
\end{tabular}

Notes: Simulated market outcomes across three integration scenarios. See Table 4 for details. 
Table A.7: Simulated Market Outcomes for RSNs (3/6)

\begin{tabular}{|c|c|c|c|c|c|c|}
\hline & & \multirow[t]{2}{*}{ (i) No VI } & \multicolumn{2}{|c|}{ (ii) VI PARs } & \multicolumn{2}{|c|}{ (iii) VI No PARs } \\
\hline & & & \multicolumn{2}{|c|}{ (vs. No VI) } & \multicolumn{2}{|c|}{ (vs. No VI) } \\
\hline & & $\overline{\text { Level }}$ & $\% \Delta_{l v l}$ & $\% \Delta_{W T P}$ & $\% \Delta_{l v l}$ & $\% \Delta_{W T P}$ \\
\hline \multicolumn{7}{|c|}{ SATELLITE OWNED RSNS } \\
\hline ROOT NW & Cable Mkt Share & 0.61 & $0.7 \%$ & & $0.6 \%$ & \\
\hline DirecTV & & {$[0.58,0.62]$} & {$[-0.4 \%, 2.8 \%]$} & & {$[-0.6 \%, 2.6 \%]$} & \\
\hline Pop $4.15 \mathrm{M}$ & Sat Mkt Share & 0.23 & $-0.5 \%$ & & $-0.4 \%$ & \\
\hline \multirow[t]{5}{*}{ WTP $\$ 1.79$} & & {$[0.22,0.23]$} & {$[-2.3 \%, 0.4 \%]$} & & {$[-2.1 \%, 1.1 \%]$} & \\
\hline & Cable Carriage & 0.78 & $0.0 \%$ & & $0.0 \%$ & \\
\hline & & {$[0.52,1.00]$} & {$[-13.4 \%, 14.2 \%]$} & & {$[-31.3 \%, 4.9 \%]$} & \\
\hline & Cable Prices & 52.90 & $-0.4 \%$ & & $-0.4 \%$ & \\
\hline & & {$[51.94,54.61]$} & {$[-1.9 \%, 0.3 \%]$} & & {$[-2.0 \%, 0.4 \%]$} & \\
\hline Foreclose: $0 \%$ & Aff Fees to Rivals & 0.75 & $1.1 \%$ & & $6.4 \%$ & \\
\hline & & {$[0.26,0.98]$} & {$[-7.8 \%, 3.1 \%]$} & & {$[-3.4 \%, 11.3 \%]$} & \\
\hline & Cable Surplus & 21.94 & $0.0 \%$ & $-0.3 \%$ & $-0.2 \%$ & $-2.4 \%$ \\
\hline & & {$[9.99,25.20]$} & {$[-0.2 \%, 0.2 \%]$} & {$[-2.1 \%, 2.2 \%]$} & {$[-0.6 \%, 0.2 \%]$} & {$[-5.8 \%, 2.3 \%]$} \\
\hline & Satellite + RSN Surplus & 6.69 & $-0.4 \%$ & $-1.4 \%$ & $0.2 \%$ & $0.9 \%$ \\
\hline & & {$[2.25,7.14]$} & {$[-2.3 \%, 1.1 \%]$} & {$[-7.7 \%, 3.3 \%]$} & {$[-3.1 \%, 1.0 \%]$} & {$[-10.9 \%, 3.0 \%]$} \\
\hline & Consumer Welfare & 33.05 & $0.4 \%$ & $7.6 \%$ & $0.4 \%$ & $6.7 \%$ \\
\hline & & {$[17.39,36.63]$} & {$[-0.2 \%, 1.7 \%]$} & {$[-4.8 \%, 31.3 \%]$} & {$[-0.9 \%, 1.6 \%]$} & {$[-15.6 \%, 28.9 \%]$} \\
\hline & Total Welfare & 61.68 & $0.2 \%$ & $5.9 \%$ & $0.2 \%$ & $5.2 \%$ \\
\hline & & {$[29.41,67.77]$} & {$[-0.3 \%, 0.8 \%]$} & {$[-9.1 \%, 27.5 \%]$} & {$[-0.7 \%, 0.7 \%]$} & {$[-23.7 \%, 24.9 \%]$} \\
\hline ROOT PITT & Cable Mkt Share & 0.62 & $1.6 \%$ & & $1.1 \%$ & \\
\hline DirecTV & & {$[0.60,0.63]$} & {$[0.3 \%, 2.4 \%]$} & & {$[-0.1 \%, 2.1 \%]$} & \\
\hline Pop $5.09 \mathrm{M}$ & Sat Mkt Share & 0.16 & $-1.9 \%$ & & $-1.2 \%$ & \\
\hline WTP $\$ 2.17$ & & {$[0.16,0.16]$} & {$[-2.2 \%,-0.2 \%]$} & & {$[-1.8 \%, 0.7 \%]$} & \\
\hline & Cable Carriage & 0.62 & $0.0 \%$ & & $-4.3 \%$ & \\
\hline & & {$[0.51,0.69]$} & {$[-22.1 \%, 5.0 \%]$} & & {$[-29.4 \%, 0.0 \%]$} & \\
\hline & Cable Prices & 55.64 & $-1.2 \%$ & & $-1.0 \%$ & \\
\hline & & {$[54.70,56.76]$} & {$[-1.8 \%,-0.2 \%]$} & & {$[-1.9 \%,-0.1 \%]$} & \\
\hline Foreclose: $0 \%$ & Aff Fees to Rivals & 1.24 & $-0.1 \%$ & & $8.4 \%$ & \\
\hline & & {$[0.53,1.51]$} & {$[-2.2 \%, 6.2 \%]$} & & {$[5.1 \%, 19.7 \%]$} & \\
\hline & Cable Surplus & 23.54 & $0.0 \%$ & $-0.1 \%$ & $-0.3 \%$ & $-3.0 \%$ \\
\hline & & {$[10.87,25.69]$} & {$[-0.4 \%, 0.0 \%]$} & {$[-4.2 \%, 0.4 \%]$} & {$[-0.7 \%,-0.1 \%]$} & {$[-7.2 \%,-1.1 \%]$} \\
\hline & Satellite + RSN Surplus & 5.04 & $-1.4 \%$ & $-3.3 \%$ & $-0.1 \%$ & $-0.1 \%$ \\
\hline & & {$[1.51,5.39]$} & {$[-4.5 \%, 0.0 \%]$} & {$[-9.4 \%, 0.1 \%]$} & {$[-5.1 \%, 1.0 \%]$} & {$[-8.4 \%, 2.3 \%]$} \\
\hline & Consumer Welfare & 28.01 & $1.1 \%$ & $14.5 \%$ & $0.8 \%$ & $10.7 \%$ \\
\hline & & {$[14.67,31.16]$} & {$[0.2 \%, 1.8 \%]$} & {$[2.5 \%, 25.4 \%]$} & {$[-0.1 \%, 1.6 \%]$} & {$[-1.0 \%, 22.2 \%]$} \\
\hline & Total Welfare & 56.59 & $0.4 \%$ & $11.1 \%$ & $0.3 \%$ & $7.6 \%$ \\
\hline & & {$[27.15,61.63]$} & {$[-0.2 \%, 0.6 \%]$} & {$[-4.5 \%, 16.5 \%]$} & {$[-0.4 \%, 0.5 \%]$} & {$[-9.8 \%, 13.2 \%]$} \\
\hline ROOT ROCKY MTN & Cable Mkt Share & 0.50 & $0.0 \%$ & & $1.0 \%$ & \\
\hline DirecTV & & {$[0.44,0.53]$} & {$[-5.1 \%, 0.0 \%]$} & & {$[-7.0 \%, 2.9 \%]$} & \\
\hline Pop $4.19 \mathrm{M}$ & Sat Mkt Share & 0.30 & $0.0 \%$ & & $-0.3 \%$ & \\
\hline WTP $\$ 1.79$ & & {$[0.29,0.33]$} & {$[0.0 \%, 4.5 \%]$} & & {$[-1.0 \%, 5.6 \%]$} & \\
\hline & Cable Carriage & 0.59 & $0.0 \%$ & & $-17.3 \%$ & \\
\hline & & {$[0.48,0.84]$} & {$[-27.5 \%, 0.0 \%]$} & & {$[-37.0 \%, 0.0 \%]$} & \\
\hline & Cable Prices & 55.84 & $0.0 \%$ & & $-0.7 \%$ & \\
\hline & & {$[54.03,59.28]$} & {$[-0.2 \%, 2.4 \%]$} & & {$[-1.5 \%, 3.1 \%]$} & \\
\hline Foreclose: $0 \%$ & Aff Fees to Rivals & 0.96 & $0.3 \%$ & & $7.9 \%$ & \\
\hline & & {$[0.26,1.29]$} & {$[-0.5 \%, 15.5 \%]$} & & {$[4.6 \%, 28.7 \%]$} & \\
\hline & Cable Surplus & 13.89 & $0.0 \%$ & $-0.1 \%$ & $-0.4 \%$ & $-3.1 \%$ \\
\hline & & {$[6.45,15.15]$} & {$[-0.8 \%, 0.0 \%]$} & {$[-5.6 \%, 0.2 \%]$} & {$[-1.4 \%,-0.2 \%]$} & {$[-10.2 \%,-0.7 \%]$} \\
\hline & Satellite + RSN Surplus & 8.90 & $0.0 \%$ & $0.2 \%$ & $-0.9 \%$ & $-4.3 \%$ \\
\hline & & {$[3.10,9.44]$} & {$[-1.2 \%, 3.6 \%]$} & {$[-4.0 \%, 16.9 \%]$} & {$[-2.7 \%, 4.8 \%]$} & {$[-13.9 \%, 22.8 \%]$} \\
\hline & Consumer Welfare & 32.59 & $0.0 \%$ & $-0.4 \%$ & $0.3 \%$ & $4.9 \%$ \\
\hline & & {$[16.28,35.90]$} & {$[-2.5 \%, 0.0 \%]$} & {$[-55.1 \%, 0.3 \%]$} & {$[-2.7 \%, 0.9 \%]$} & {$[-63.3 \%, 19.7 \%]$} \\
\hline & Total Welfare & $\begin{array}{r}55.38 \\
{[10.20,58}\end{array}$ & $0.0 \%$ & $-0.3 \%$ & $-0.1 \%$ & $-2.4 \%$ \\
\hline & & {$[25.12,60.28]$} & {$[-1.1 \%, 0.0 \%]$} & {$[-47.3 \%, 0.0 \%]$} & {$[-1.3 \%, 0.0 \%]$} & {$[-50.2 \%, 0.1 \%]$} \\
\hline NON-INTEGRATE & RSNs (1/4) & & & & & \\
\hline ALTITUDE & Cable Mkt Share & 0.54 & $0.2 \%$ & & $0.1 \%$ & \\
\hline *Comcast & & {$[0.48,0.57]$} & {$[-0.9 \%, 6.1 \%]$} & & {$[-1.0 \%, 5.2 \%]$} & \\
\hline Pop $7.12 \mathrm{M}$ & Sat Mkt Share & 0.28 & $-0.1 \%$ & & $-0.1 \%$ & \\
\hline Footprint $74 \%$ & & {$[0.26,0.30]$} & {$[-5.9 \%, 0.3 \%]$} & & {$[-5.2 \%, 0.4 \%]$} & \\
\hline WTP $\$ 3.22$ & Cable Carriage & 0.48 & $0.0 \%$ & & $0.0 \%$ & \\
\hline & & {$[0.23,0.60]$} & {$[0.0 \%, 213.0 \%]$} & & {$[0.0 \%, 213.0 \%]$} & \\
\hline & Cable Prices & 56.41 & $-0.1 \%$ & & $-0.1 \%$ & \\
\hline & & {$[54.26,59.69]$} & {$[-2.8 \%, 1.2 \%]$} & & {$[-2.6 \%, 1.2 \%]$} & \\
\hline Foreclose: $1 \%$ & Aff Fees to Sat & 1.20 & $5.0 \%$ & & $33.8 \%$ & \\
\hline & & {$[0.39,1.48]$} & {$[-21.2 \%, 10.8 \%]$} & & {$[2.0 \%, 52.8 \%]$} & \\
\hline & Cable + RSN Surplus & $\begin{array}{r}{[0.07,1.40]} \\
16.97\end{array}$ & $0.1 \%$ & $0.6 \%$ & $0.7 \%$ & $3.5 \%$ \\
\hline & & {$[8.43,18.54]$} & {$[0.1 \%, 2.4 \%]$} & {$[0.4 \%, 13.2 \%]$} & {$[0.5 \%, 2.9 \%]$} & {$[2.7 \%, 16.5 \%]$} \\
\hline & Satellite Surplus & 6.97 & $-0.4 \%$ & $-0.8 \%$ & $-1.7 \%$ & $-3.6 \%$ \\
\hline & & {$[2.12,7.54]$} & {$[-5.8 \%, 0.2 \%]$} & {$[-10.9 \%, 0.4 \%]$} & {$[-5.4 \%,-0.6 \%]$} & {$[-12.1 \%,-0.8 \%]$} \\
\hline & Consumer Welfare & 32.60 & $0.1 \%$ & $0.8 \%$ & $0.0 \%$ & $0.4 \%$ \\
\hline & & {$[16.47,36.56]$} & {$[-0.2 \%, 3.0 \%]$} & {$[-2.6 \%, 28.9 \%]$} & {$[-1.0 \%, 2.3 \%]$} & {$[-10.0 \%, 26.2 \%]$} \\
\hline & Total Welfare & 56.54 & $0.0 \%$ & $0.6 \%$ & $0.0 \%$ & $0.3 \%$ \\
\hline & & {$[26.28,62.37]$} & {$[-0.1 \%, 1.4 \%]$} & {$[-1.2 \%, 23.3 \%]$} & {$[-0.4 \%, 1.1 \%]$} & {$[-6.3 \%, 20.7 \%]$} \\
\hline
\end{tabular}

Notes: Simulated market outcomes across three integration scenarios. "Satellite + RSN Surplus" includes profits for both DirecTV and Dish. See Table 4 for details. 
Table A.8: Simulated Market Outcomes for RSNs (4/6)

\begin{tabular}{|c|c|c|c|c|c|c|}
\hline & & \multirow[t]{2}{*}{ (i) No VI } & \multicolumn{2}{|c|}{ (ii) VI PARs } & \multicolumn{2}{|c|}{ (iii) VI No PARs } \\
\hline & & & \multicolumn{2}{|c|}{ (vs. No VI) } & \multicolumn{2}{|c|}{ (vs. No VI) } \\
\hline & & Level & $\% \Delta_{l v l}$ & $\% \Delta_{W T P}$ & $\% \Delta_{l v l}$ & $\% \Delta_{W T P}$ \\
\hline \multicolumn{7}{|c|}{ NON-INTEGRATED RSNs (2/4) } \\
\hline FS DETROIT & Cable Mkt Share & 0.56 & $3.1 \%$ & & $3.0 \%$ & \\
\hline${ }^{*}$ Comcast & & {$[0.54,0.58]$} & {$[0.3 \%, 6.4 \%]$} & & {$[0.2 \%, 6.3 \%]$} & \\
\hline Pop $4.84 \mathrm{M}$ & Sat Mkt Share & 0.17 & $-3.7 \%$ & & $-3.6 \%$ & \\
\hline Footprint $82 \%$ & & {$[0.17,0.18]$} & {$[-7.1 \%,-0.4 \%]$} & & {$[-11.8 \%,-0.3 \%]$} & \\
\hline \multirow[t]{4}{*}{ WTP $\$ 4.38$} & Cable Carriage & 0.76 & $27.6 \%$ & & $27.6 \%$ & \\
\hline & & {$[0.55,0.87]$} & {$[6.7 \%, 77.4 \%]$} & & {$[6.7 \%, 77.4 \%]$} & \\
\hline & Cable Prices & 50.34 & $-1.0 \%$ & & $-1.0 \%$ & \\
\hline & & {$[49.71,51.44]$} & {$[-3.0 \%, 0.1 \%]$} & & {$[-2.8 \%, 0.5 \%]$} & \\
\hline \multirow{10}{*}{ Foreclose: $7 \%$} & Aff Fees to Sat & 2.10 & $-11.4 \%$ & & $12.7 \%$ & \\
\hline & & {$[0.76,2.69]$} & {$[-30.7 \%, 2.4 \%]$} & & {$[-14.9 \%, 33.3 \%]$} & \\
\hline & Cable + RSN Surplus & 20.37 & $1.2 \%$ & $5.6 \%$ & $1.6 \%$ & $7.5 \%$ \\
\hline & & {$[9.70,22.30]$} & {$[0.3 \%, 2.5 \%]$} & {$[1.8 \%, 11.5 \%]$} & {$[0.7 \%, 3.1 \%]$} & {$[3.7 \%, 13.0 \%]$} \\
\hline & Satellite Surplus & 4.38 & $-2.8 \%$ & $-2.8 \%$ & $-4.6 \%$ & $-4.6 \%$ \\
\hline & & {$[1.33,4.74]$} & {$[-4.9 \%,-0.3 \%]$} & {$[-4.8 \%,-0.4 \%]$} & {$[-6.8 \%,-1.4 \%]$} & {$[-6.3 \%,-1.8 \%]$} \\
\hline & Consumer Welfare & 26.64 & $2.4 \%$ & $14.4 \%$ & $2.3 \%$ & $13.9 \%$ \\
\hline & & {$[13.84,29.68]$} & {$[0.2 \%, 5.2 \%]$} & {$[1.4 \%, 30.8 \%]$} & {$[-1.5 \%, 5.0 \%]$} & {$[-9.2 \%, 30.2 \%]$} \\
\hline & Total Welfare & 51.38 & $1.5 \%$ & $17.2 \%$ & $1.4 \%$ & $16.8 \%$ \\
\hline & & {$[24.53,56.31]$} & {$[0.2 \%, 3.3 \%]$} & {$[3.7 \%, 38.5 \%]$} & {$[-0.2 \%, 3.2 \%]$} & {$[-2.7 \%, 36.6 \%]$} \\
\hline FS FLORIDA & Cable Mkt Share & 0.60 & $0.8 \%$ & & $0.7 \%$ & \\
\hline$*$ Comcast & & {$[0.59,0.61]$} & {$[0.1 \%, 3.4 \%]$} & & {$[-0.1 \%, 3.1 \%]$} & \\
\hline Pop 6.20M & Sat Mkt Share & 0.23 & $-0.8 \%$ & & $-0.7 \%$ & \\
\hline Footprint $67 \%$ & & {$[0.22,0.23]$} & {$[-3.7 \%,-0.1 \%]$} & & {$[-9.6 \%, 0.0 \%]$} & \\
\hline WTP $\$ 2.59$ & Cable Carriage & 0.90 & $2.2 \%$ & & $2.2 \%$ & \\
\hline & & {$[0.53,0.94]$} & {$[0.5 \%, 68.6 \%]$} & & {$[0.0 \%, 68.6 \%]$} & \\
\hline & Cable Prices & 54.19 & $-0.4 \%$ & & $-0.3 \%$ & \\
\hline & & {$[53.95,54.93]$} & {$[-1.4 \%, 0.2 \%]$} & & {$[-1.1 \%, 1.0 \%]$} & \\
\hline Foreclose: $9 \%$ & Aff Fees to Sat & 1.47 & $-0.7 \%$ & & $25.8 \%$ & \\
\hline & & {$[0.64,1.71]$} & {$[-14.1 \%, 5.5 \%]$} & & {$[3.3 \%, 40.1 \%]$} & \\
\hline & Cable + RSN Surplus & 19.84 & $0.1 \%$ & $0.9 \%$ & $0.6 \%$ & $4.4 \%$ \\
\hline & & {$[8.90,21.98]$} & {$[0.1 \%, 1.6 \%]$} & {$[0.5 \%, 13.0 \%]$} & {$[0.3 \%, 2.1 \%]$} & {$[3.5 \%, 16.5 \%]$} \\
\hline & Satellite Surplus & 6.08 & $-0.8 \%$ & $-1.9 \%$ & $-2.2 \%$ & $-5.2 \%$ \\
\hline & & {$[1.79,6.69]$} & {$[-3.3 \%,-0.2 \%]$} & {$[-9.7 \%,-0.5 \%]$} & {$[-4.5 \%,-0.6 \%]$} & {$[-12.8 \%,-1.3 \%]$} \\
\hline & Consumer Welfare & 31.20 & $0.5 \%$ & $5.5 \%$ & $0.4 \%$ & $4.6 \%$ \\
\hline & & {$[15.35,34.16]$} & {$[0.1 \%, 2.4 \%]$} & {$[1.2 \%, 35.4 \%]$} & {$[-2.7 \%, 2.2 \%]$} & {$[-33.2 \%, 32.5 \%]$} \\
\hline & Total Welfare & 57.11 & $0.2 \%$ & $4.5 \%$ & $0.2 \%$ & $3.9 \%$ \\
\hline & & {$[24.93,62.59]$} & {$[0.1 \%, 1.3 \%]$} & {$[1.3 \%, 37.2 \%]$} & {$[-1.2 \%, 1.3 \%]$} & {$[-25.1 \%, 35.6 \%]$} \\
\hline FS MIDWEST & Cable Mkt Share & 0.60 & $1.8 \%$ & & $1.7 \%$ & \\
\hline${ }^{*}$ Comcast & & {$[0.59,0.62]$} & {$[0.1 \%, 2.1 \%]$} & & {$[0.1 \%, 2.1 \%]$} & \\
\hline Pop 10.40M & Sat Mkt Share & 0.21 & $-1.2 \%$ & & $-1.2 \%$ & \\
\hline Footprint $26 \%$ & & {$[0.21,0.21]$} & {$[-1.5 \%, 0.0 \%]$} & & {$[-1.5 \%, 0.1 \%]$} & \\
\hline WTP $\$ 2.44$ & Cable Carriage & 0.43 & $29.5 \%$ & & $29.5 \%$ & \\
\hline & & {$[0.26,0.55]$} & {$[3.3 \%, 51.4 \%]$} & & {$[-13.9 \%, 50.4 \%]$} & \\
\hline & Cable Prices & 52.15 & $-0.8 \%$ & & $-0.7 \%$ & \\
\hline & & {$[51.19,52.45]$} & {$[-1.0 \%, 0.1 \%]$} & & {$[-1.0 \%, 0.1 \%]$} & \\
\hline Foreclose: $0 \%$ & Aff Fees to Sat & 1.18 & $-1.5 \%$ & & $17.1 \%$ & \\
\hline & & {$[0.55,1.31]$} & {$[-6.5 \%, 3.1 \%]$} & & {$[10.5 \%, 31.9 \%]$} & \\
\hline & Cable + RSN Surplus & 19.92 & $0.3 \%$ & $2.8 \%$ & $0.6 \%$ & $4.7 \%$ \\
\hline & & {$[9.44,21.77]$} & {$[0.0 \%, 0.6 \%]$} & {$[0.0 \%, 4.8 \%]$} & {$[-0.1 \%, 0.7 \%]$} & {$[-0.9 \%, 6.8 \%]$} \\
\hline & Satellite Surplus & 5.26 & $-1.2 \%$ & $-2.6 \%$ & $-2.0 \%$ & $-4.4 \%$ \\
\hline & & {$[1.69,5.66]$} & {$[-1.4 \%, 0.0 \%]$} & {$[-2.7 \%,-0.2 \%]$} & {$[-2.5 \%,-0.7 \%]$} & {$[-4.7 \%,-1.9 \%]$} \\
\hline & Consumer Welfare & 29.92 & $1.3 \%$ & $15.8 \%$ & $1.3 \%$ & $15.6 \%$ \\
\hline & & {$[15.57,33.40]$} & {$[0.1 \%, 1.4 \%]$} & {$[1.2 \%, 16.7 \%]$} & {$[0.0 \%, 1.4 \%]$} & {$[0.1 \%, 16.6 \%]$} \\
\hline & Total Welfare & 55.10 & $0.7 \%$ & $16.0 \%$ & $0.7 \%$ & $15.9 \%$ \\
\hline & & {$[26.21,60.16]$} & {$[0.1 \%, 0.8 \%]$} & {$[1.3 \%, 16.6 \%]$} & {$[-0.1 \%, 0.8 \%]$} & {$[-2.5 \%, 16.5 \%]$} \\
\hline FS NORTH & Cable Mkt Share & 0.61 & $0.1 \%$ & & $0.1 \%$ & \\
\hline$*$ Charter & & {$[0.59,0.64]$} & {$[0.0 \%, 0.7 \%]$} & & {$[0.0 \%, 0.7 \%]$} & \\
\hline Pop 5.77M & Sat Mkt Share & 0.15 & $-0.2 \%$ & & $-0.2 \%$ & \\
\hline Footprint $12 \%$ & & {$[0.14,0.16]$} & {$[-1.4 \%, 0.0 \%]$} & & {$[-1.4 \%, 0.0 \%]$} & \\
\hline WTP $\$ 4.50$ & Cable Carriage & 0.87 & $0.0 \%$ & & $0.0 \%$ & \\
\hline & & {$[0.62,0.90]$} & {$[0.0 \%, 7.4 \%]$} & & {$[0.0 \%, 7.1 \%]$} & \\
\hline & Cable Prices & 52.04 & $-0.1 \%$ & & $-0.1 \%$ & \\
\hline & & {$[50.73,53.33]$} & {$[-0.3 \%, 0.1 \%]$} & & {$[-0.3 \%, 0.1 \%]$} & \\
\hline Foreclose: $0 \%$ & Aff Fees to Sat & 2.42 & $0.5 \%$ & & $7.7 \%$ & \\
\hline & & {$[0.89,3.09]$} & {$[-7.1 \%, 1.7 \%]$} & & {$[-0.4 \%, 12.6 \%]$} & \\
\hline & Cable + RSN Surplus & 23.02 & $0.0 \%$ & $0.1 \%$ & $0.1 \%$ & $0.6 \%$ \\
\hline & & {$[10.75,25.23]$} & {$[0.0 \%, 0.4 \%]$} & {$[0.0 \%, 1.8 \%]$} & {$[0.1 \%, 0.4 \%]$} & {$[0.6 \%, 2.0 \%]$} \\
\hline & Satellite Surplus & 3.71 & $-0.2 \%$ & $-0.2 \%$ & $-0.9 \%$ & $-0.8 \%$ \\
\hline & & {$[1.22,4.00]$} & {$[-1.1 \%, 0.0 \%]$} & {$[-0.8 \%, 0.0 \%]$} & {$[-2.0 \%,-0.5 \%]$} & {$[-1.4 \%,-0.6 \%]$} \\
\hline & Consumer Welfare & 27.70 & $0.1 \%$ & $0.6 \%$ & $0.1 \%$ & $0.6 \%$ \\
\hline & & {$[14.35,31.04]$} & {$[0.0 \%, 0.6 \%]$} & {$[0.0 \%, 3.7 \%]$} & {$[0.0 \%, 0.6 \%]$} & {$[-0.1 \%, 3.6 \%]$} \\
\hline & Total Welfare & 54.43 & $0.0 \%$ & $0.5 \%$ & $0.0 \%$ & $0.5 \%$ \\
\hline & & {$[25.87,59.69]$} & {$[0.0 \%, 0.3 \%]$} & {$[0.0 \%, 4.5 \%]$} & {$[0.0 \%, 0.3 \%]$} & {$[-0.1 \%, 3.9 \%]$} \\
\hline
\end{tabular}

Notes: Simulated market outcomes across three integration scenarios. See Table 4 for details. 
Table A.9: Simulated Market Outcomes for RSNs (5/6)

\begin{tabular}{|c|c|c|c|c|c|c|}
\hline & & \multirow[t]{2}{*}{ (i) No VI } & \multicolumn{2}{|c|}{ (ii) VI PARs } & \multicolumn{2}{|c|}{ (iii) VI No PARs } \\
\hline & & & \multicolumn{2}{|c|}{ (vs. No VI) } & \multicolumn{2}{|c|}{ (vs. No VI) } \\
\hline & & Level & $\% \Delta_{l v l}$ & $\% \Delta_{W T P}$ & $\% \Delta_{l v l}$ & $\% \Delta_{W T P}$ \\
\hline \multicolumn{7}{|c|}{ NON-INTEGRATED RSNs (3/4) } \\
\hline FS OHIO & Cable Mkt Share & 0.60 & $2.0 \%$ & & $2.0 \%$ & \\
\hline${ }^{*} \mathrm{TWC}$ & & {$[0.60,0.62]$} & {$[0.1 \%, 2.4 \%]$} & & {$[0.1 \%, 2.4 \%]$} & \\
\hline Pop 8.16M & Sat Mkt Share & 0.18 & $-1.7 \%$ & & $-1.7 \%$ & \\
\hline Footprint $51 \%$ & & {$[0.18,0.18]$} & {$[-2.5 \%,-0.1 \%]$} & & {$[-2.6 \%, 0.0 \%]$} & \\
\hline \multirow[t]{4}{*}{ WTP $\$ 3.70$} & Cable Carriage & 0.72 & $5.3 \%$ & & $5.3 \%$ & \\
\hline & & {$[0.41,0.78]$} & {$[0.0 \%, 46.0 \%]$} & & {$[0.0 \%, 46.0 \%]$} & \\
\hline & Cable Prices & 52.43 & $-1.2 \%$ & & $-1.2 \%$ & \\
\hline & & {$[51.32,52.52]$} & {$[-1.3 \%, 0.2 \%]$} & & {$[-1.2 \%, 0.3 \%]$} & \\
\hline \multirow{10}{*}{ Foreclose: $1 \%$} & Aff Fees to Sat & 1.72 & $2.4 \%$ & & $20.1 \%$ & \\
\hline & & {$[0.75,1.94]$} & {$[-7.1 \%, 8.8 \%]$} & & {$[5.4 \%, 32.3 \%]$} & \\
\hline & Cable + RSN Surplus & 22.39 & $0.3 \%$ & $1.6 \%$ & $0.5 \%$ & $3.0 \%$ \\
\hline & & {$[10.30,25.14]$} & {$[0.0 \%, 1.4 \%]$} & {$[0.3 \%, 9.1 \%]$} & {$[0.3 \%, 1.7 \%]$} & {$[1.7 \%, 10.8 \%]$} \\
\hline & Satellite Surplus & 4.47 & $-1.9 \%$ & $-2.2 \%$ & $-3.1 \%$ & $-3.7 \%$ \\
\hline & & {$[1.39,4.76]$} & {$[-2.3 \%,-0.2 \%]$} & {$[-2.7 \%,-0.2 \%]$} & {$[-4.0 \%,-0.6 \%]$} & {$[-3.9 \%,-0.8 \%]$} \\
\hline & Consumer Welfare & 28.65 & $1.4 \%$ & $10.7 \%$ & $1.4 \%$ & $10.5 \%$ \\
\hline & & {$[15.23,32.22]$} & {$[0.1 \%, 2.2 \%]$} & {$[0.7 \%, 17.3 \%]$} & {$[0.0 \%, 2.1 \%]$} & {$[0.3 \%, 16.0 \%]$} \\
\hline & Total Welfare & 55.51 & $0.7 \%$ & $10.0 \%$ & $0.7 \%$ & $9.9 \%$ \\
\hline & & {$[26.41,61.52]$} & {$[0.1 \%, 1.4 \%]$} & {$[0.8 \%, 23.9 \%]$} & {$[0.0 \%, 1.4 \%]$} & {$[0.7 \%, 22.4 \%]$} \\
\hline FS SOUTH & Cable Mkt Share & 0.62 & $0.3 \%$ & & $0.3 \%$ & \\
\hline${ }^{*} \mathrm{TWC}$ & & {$[0.61,0.63]$} & {$[-0.1 \%, 1.0 \%]$} & & {$[-0.2 \%, 1.0 \%]$} & \\
\hline Pop $13.20 \mathrm{M}$ & Sat Mkt Share & 0.21 & $-0.7 \%$ & & $-0.7 \%$ & \\
\hline Footprint $33 \%$ & & {$[0.21,0.22]$} & {$[-1.3 \%, 0.2 \%]$} & & {$[-1.3 \%, 0.3 \%]$} & \\
\hline WTP $\$ 1.63$ & Cable Carriage & 0.83 & $12.1 \%$ & & $12.1 \%$ & \\
\hline & & {$[0.58,0.94]$} & {$[-7.0 \%, 37.3 \%]$} & & {$[-7.1 \%, 37.3 \%]$} & \\
\hline & Cable Prices & 55.63 & $0.0 \%$ & & $0.0 \%$ & \\
\hline & & {$[55.40,56.48]$} & {$[-0.6 \%, 0.3 \%]$} & & {$[-0.6 \%, 0.3 \%]$} & \\
\hline Foreclose: $0 \%$ & Aff Fees to Sat & 0.87 & $-7.2 \%$ & & $5.2 \%$ & \\
\hline & & {$[0.28,0.96]$} & {$[-7.1 \%, 5.6 \%]$} & & {$[-0.2 \%, 23.1 \%]$} & \\
\hline & Cable + RSN Surplus & 22.29 & $0.3 \%$ & $4.0 \%$ & $0.4 \%$ & $5.5 \%$ \\
\hline & & {$[10.89,24.52]$} & {$[-0.2 \%, 0.6 \%]$} & {$[-2.8 \%, 9.1 \%]$} & {$[-0.1 \%, 0.7 \%]$} & {$[-2.9 \%, 10.5 \%]$} \\
\hline & Satellite Surplus & 5.76 & $-0.4 \%$ & $-1.6 \%$ & $-0.8 \%$ & $-3.0 \%$ \\
\hline & & {$[1.80,6.25]$} & {$[-1.0 \%, 0.2 \%]$} & {$[-3.7 \%, 0.6 \%]$} & {$[-1.4 \%,-0.2 \%]$} & {$[-5.0 \%,-0.8 \%]$} \\
\hline & Consumer Welfare & 30.70 & $0.3 \%$ & $5.9 \%$ & $0.3 \%$ & $5.7 \%$ \\
\hline & & {$[15.81,33.88]$} & {$[0.0 \%, 0.8 \%]$} & {$[-0.6 \%, 15.9 \%]$} & {$[0.0 \%, 0.8 \%]$} & {$[-0.8 \%, 15.8 \%]$} \\
\hline & Total Welfare & 58.75 & $0.2 \%$ & $8.3 \%$ & $0.2 \%$ & $8.2 \%$ \\
\hline & & {$[26.82,64.29]$} & {$[-0.1 \%, 0.6 \%]$} & {$[-2.2 \%, 23.3 \%]$} & {$[-0.1 \%, 0.6 \%]$} & {$[-3.9 \%, 23.2 \%]$} \\
\hline FS SOUTHWEST & Cable Mkt Share & 0.57 & $2.5 \%$ & & $2.5 \%$ & \\
\hline${ }^{*} \mathrm{Cox}$ & & {$[0.56,0.59]$} & {$[0.3 \%, 4.1 \%]$} & & {$[0.3 \%, 4.1 \%]$} & \\
\hline Pop $12.70 \mathrm{M}$ & Sat Mkt Share & 0.23 & $-1.1 \%$ & & $-1.1 \%$ & \\
\hline Footprint $37 \%$ & & {$[0.22,0.23]$} & {$[-2.8 \%,-0.2 \%]$} & & {$[-2.6 \%,-0.1 \%]$} & \\
\hline WTP $\$ 3.07$ & Cable Carriage & 1.00 & $0.0 \%$ & & $0.0 \%$ & \\
\hline & & {$[0.71,1.00]$} & {$[-6.3 \%, 28.8 \%]$} & & {$[-16.7 \%, 22.3 \%]$} & \\
\hline & Cable Prices & 51.20 & $-1.3 \%$ & & $-1.3 \%$ & \\
\hline & & {$[49.89,51.38]$} & {$[-2.0 \%, 0.0 \%]$} & & {$[-2.4 \%,-0.1 \%]$} & \\
\hline Foreclose: $0 \%$ & Aff Fees to Sat & 1.56 & $0.1 \%$ & & $8.4 \%$ & \\
\hline & & {$[0.62,1.74]$} & {$[-10.4 \%, 3.7 \%]$} & & {$[0.1 \%, 21.4 \%]$} & \\
\hline & Cable + RSN Surplus & 18.87 & $0.1 \%$ & $0.5 \%$ & $0.2 \%$ & $1.4 \%$ \\
\hline & & {$[8.25,20.63]$} & {$[-0.2 \%, 1.2 \%]$} & {$[-1.5 \%, 7.5 \%]$} & {$[-0.8 \%, 1.0 \%]$} & {$[-5.0 \%, 5.9 \%]$} \\
\hline & Satellite Surplus & 5.98 & $-1.1 \%$ & $-2.2 \%$ & $-1.6 \%$ & $-3.2 \%$ \\
\hline & & {$[1.90,6.39]$} & {$[-2.2 \%,-0.2 \%]$} & {$[-4.5 \%,-0.4 \%]$} & {$[-2.9 \%,-0.5 \%]$} & {$[-5.1 \%,-1.2 \%]$} \\
\hline & Consumer Welfare & 29.80 & $1.3 \%$ & $12.3 \%$ & $1.3 \%$ & $12.2 \%$ \\
\hline & & {$[14.95,33.03]$} & {$[0.2 \%, 2.3 \%]$} & {$[1.7 \%, 21.4 \%]$} & {$[0.1 \%, 2.2 \%]$} & {$[1.4 \%, 21.1 \%]$} \\
\hline & Total Welfare & 54.64 & $0.6 \%$ & $10.6 \%$ & $0.6 \%$ & $10.5 \%$ \\
\hline & & {$[24.40,59.98]$} & {$[0.1 \%, 1.3 \%]$} & {$[1.3 \%, 23.7 \%]$} & {$[0.0 \%, 1.1 \%]$} & {$[-0.7 \%, 19.2 \%]$} \\
\hline FS WEST & Cable Mkt Share & 0.55 & $5.8 \%$ & & $5.7 \%$ & \\
\hline *TWC & & {$[0.51,0.56]$} & {$[0.5 \%, 7.7 \%]$} & & {$[0.5 \%, 7.6 \%]$} & \\
\hline Pop 8.43M & Sat Mkt Share & 0.25 & $-4.2 \%$ & & $-4.2 \%$ & \\
\hline Footprint $53 \%$ & & {$[0.24,0.26]$} & {$[-5.9 \%,-0.3 \%]$} & & {$[-6.2 \%,-0.2 \%]$} & \\
\hline WTP $\$ 4.06$ & Cable Carriage & 0.81 & $10.0 \%$ & & $10.0 \%$ & \\
\hline & & {$[0.63,0.99]$} & {$[-10.8 \%, 37.4 \%]$} & & {$[-10.8 \%, 37.4 \%]$} & \\
\hline & Cable Prices & 53.50 & $-2.9 \%$ & & $-2.9 \%$ & \\
\hline & & {$[52.57,55.80]$} & {$[-3.9 \%,-0.1 \%]$} & & {$[-3.8 \%,-0.1 \%]$} & \\
\hline Foreclose: $1 \%$ & Aff Fees to Sat & 1.82 & $-2.5 \%$ & & $15.3 \%$ & \\
\hline & & {$[0.67,1.99]$} & {$[-12.5 \%, 8.8 \%]$} & & {$[-1.7 \%, 36.4 \%]$} & \\
\hline & Cable + RSN Surplus & 19.56 & $1.0 \%$ & $4.6 \%$ & $1.3 \%$ & $6.5 \%$ \\
\hline & & {$[10.03,21.49]$} & {$[-0.7 \%, 1.8 \%]$} & {$[-3.6 \%, 9.4 \%]$} & {$[-0.3 \%, 2.2 \%]$} & {$[-1.5 \%, 11.1 \%]$} \\
\hline & Satellite Surplus & 6.17 & $-4.1 \%$ & $-6.3 \%$ & $-5.3 \%$ & $-8.1 \%$ \\
\hline & & {$[1.86,6.68]$} & {$[-5.5 \%,-0.5 \%]$} & {$[-7.9 \%,-1.0 \%]$} & {$[-6.7 \%,-1.0 \%]$} & {$[-9.7 \%,-3.0 \%]$} \\
\hline & Consumer Welfare & 32.01 & $2.6 \%$ & $20.8 \%$ & $2.6 \%$ & $20.5 \%$ \\
\hline & & {$[16.15,35.45]$} & {$[0.3 \%, 3.8 \%]$} & {$[2.6 \%, 30.3 \%]$} & {$[0.3 \%, 3.7 \%]$} & {$[2.2 \%, 30.0 \%]$} \\
\hline & Total Welfare & 57.75 & $1.3 \%$ & $19.1 \%$ & $1.3 \%$ & $18.9 \%$ \\
\hline & & {$[26.58,62.69]$} & {$[0.0 \%, 1.9 \%]$} & {$[-0.5 \%, 26.9 \%]$} & {$[-0.1 \%, 1.9 \%]$} & {$[-1.7 \%, 26.8 \%]$} \\
\hline
\end{tabular}

Notes: Simulated market outcomes across three integration scenarios. See Table 4 for details. 
Table A.10: Simulated Market Outcomes for RSNs (6/6)

\begin{tabular}{|c|c|c|c|c|c|c|}
\hline & & \multirow[t]{2}{*}{ (i) No VI } & \multicolumn{2}{|c|}{ (ii) VI PARs } & \multicolumn{2}{|c|}{ (iii) VI No PARs } \\
\hline & & & \multicolumn{2}{|c|}{ (vs. No VI) } & \multicolumn{2}{|c|}{ (vs. No VI) } \\
\hline & & Level & $\% \Delta_{l v l}$ & $\% \Delta_{W T P}$ & $\% \Delta_{l v l}$ & $\% \Delta_{W T P}$ \\
\hline \multicolumn{7}{|c|}{ NON-INTEGRATED RSNS (4/4) } \\
\hline MASN & Cable Mkt Share & 0.64 & $4.5 \%$ & & $4.4 \%$ & \\
\hline${ }^{*}$ Comcast & & {$[0.61,0.66]$} & {$[1.0 \%, 5.6 \%]$} & & {$[0.9 \%, 5.5 \%]$} & \\
\hline Pop $8.25 \mathrm{M}$ & Sat Mkt Share & 0.18 & $-4.1 \%$ & & $-3.9 \%$ & \\
\hline Footprint $52 \%$ & & {$[0.17,0.18]$} & {$[-7.1 \%,-1.0 \%]$} & & {$[-10.7 \%,-1.0 \%]$} & \\
\hline \multirow[t]{4}{*}{ WTP $\$ 2.21$} & Cable Carriage & 0.51 & $10.2 \%$ & & $6.1 \%$ & \\
\hline & & {$[0.41,0.71]$} & {$[2.6 \%, 39.0 \%]$} & & {$[2.2 \%, 39.0 \%]$} & \\
\hline & Cable Prices & 56.71 & $-2.5 \%$ & & $-2.6 \%$ & \\
\hline & & {$[55.59,58.38]$} & {$[-2.9 \%,-0.1 \%]$} & & {$[-2.8 \%,-0.1 \%]$} & \\
\hline \multirow[t]{10}{*}{ Foreclose: $3 \%$} & Aff Fees to Sat & 1.47 & $0.2 \%$ & & $27.2 \%$ & \\
\hline & & {$[0.53,1.70]$} & {$[-7.4 \%, 6.8 \%]$} & & {$[13.9 \%, 38.5 \%]$} & \\
\hline & Cable + RSN Surplus & 23.52 & $0.1 \%$ & $1.0 \%$ & $0.3 \%$ & $3.3 \%$ \\
\hline & & {$[11.47,26.15]$} & {$[-0.1 \%, 0.9 \%]$} & {$[-1.6 \%, 8.5 \%]$} & {$[0.1 \%, 1.5 \%]$} & {$[1.3 \%, 16.5 \%]$} \\
\hline & Satellite Surplus & 4.44 & $-4.2 \%$ & $-8.4 \%$ & $-5.7 \%$ & $-11.4 \%$ \\
\hline & & {$[1.34,4.87]$} & {$[-6.6 \%,-0.9 \%]$} & {$[-14.0 \%,-2.6 \%]$} & {$[-7.5 \%,-1.5 \%]$} & {$[-16.8 \%,-4.6 \%]$} \\
\hline & Consumer Welfare & 29.80 & $3.4 \%$ & $45.3 \%$ & $3.3 \%$ & $44.4 \%$ \\
\hline & & {$[15.53,32.88]$} & {$[0.8 \%, 4.2 \%]$} & {$[11.3 \%, 65.2 \%]$} & {$[-0.7 \%, 4.2 \%]$} & {$[-9.9 \%, 64.3 \%]$} \\
\hline & Total Welfare & 57.76 & $1.5 \%$ & $37.9 \%$ & $1.4 \%$ & $36.3 \%$ \\
\hline & & {$[27.68,63.28]$} & {$[0.4 \%, 1.9 \%]$} & {$[12.7 \%, 51.3 \%]$} & {$[-0.1 \%, 1.9 \%]$} & {$[-1.8 \%, 51.2 \%]$} \\
\hline $\begin{array}{l}\text { PRIME TICKET } \\
*_{\text {TWC }}\end{array}$ & Cable Mkt Share & $\begin{array}{r}0.55 \\
{[0.51,0.56]}\end{array}$ & $\begin{array}{r}3.2 \% \\
{[0.4 \%, 7.1 \%]}\end{array}$ & & $\begin{array}{r}3.2 \% \\
{[0.5 \%, 6.8 \%]}\end{array}$ & \\
\hline Pop $8.32 \mathrm{M}$ & Sat Mkt Share & 0.25 & $-2.2 \%$ & & $-2.2 \%$ & \\
\hline Footprint $53 \%$ & & {$[0.24,0.26]$} & {$[-4.7 \%,-0.3 \%]$} & & {$[-4.7 \%,-0.3 \%]$} & \\
\hline \multirow[t]{4}{*}{ WTP $\$ 3.11$} & Cable Carriage & 0.79 & $8.3 \%$ & & $8.3 \%$ & \\
\hline & & {$[0.60,0.87]$} & {$[-1.0 \%, 23.8 \%]$} & & {$[-1.0 \%, 23.8 \%]$} & \\
\hline & Cable Prices & 53.29 & $-1.7 \%$ & & $-1.7 \%$ & \\
\hline & & {$[52.73,55.97]$} & {$[-3.6 \%,-0.1 \%]$} & & {$[-3.3 \%,-0.1 \%]$} & \\
\hline \multirow{10}{*}{ Foreclose: $1 \%$} & Aff Fees to Sat & 1.48 & $0.2 \%$ & & $18.3 \%$ & \\
\hline & & {$[0.59,1.70]$} & {$[-8.3 \%, 6.1 \%]$} & & {$[3.5 \%, 34.2 \%]$} & \\
\hline & Cable + RSN Surplus & 19.19 & $0.5 \%$ & $3.0 \%$ & $0.8 \%$ & $5.0 \%$ \\
\hline & & {$[9.68,21.21]$} & {$[0.0 \%, 1.0 \%]$} & {$[-0.1 \%, 6.5 \%]$} & {$[0.3 \%, 1.5 \%]$} & {$[1.8 \%, 9.7 \%]$} \\
\hline & Satellite Surplus & 6.09 & $-2.2 \%$ & $-4.3 \%$ & $-3.2 \%$ & $-6.2 \%$ \\
\hline & & {$[1.86,6.69]$} & {$[-4.6 \%,-0.4 \%]$} & {$[-9.0 \%,-1.0 \%]$} & {$[-5.7 \%,-0.9 \%]$} & {$[-10.5 \%,-2.7 \%]$} \\
\hline & Consumer Welfare & 32.33 & $1.5 \%$ & $15.5 \%$ & $1.5 \%$ & $15.2 \%$ \\
\hline & & {$[16.15,35.63]$} & {$[0.3 \%, 3.5 \%]$} & {$[3.4 \%, 34.9 \%]$} & {$[0.3 \%, 3.4 \%]$} & {$[3.1 \%, 34.6 \%]$} \\
\hline & Total Welfare & 57.61 & $0.8 \%$ & $14.2 \%$ & $0.8 \%$ & $14.1 \%$ \\
\hline & & {$[26.19,62.28]$} & {$[0.2 \%, 1.7 \%]$} & {$[3.9 \%, 29.1 \%]$} & {$[0.1 \%, 1.6 \%]$} & {$[3.5 \%, 28.7 \%]$} \\
\hline SUN SPORTS & Cable Mkt Share & 0.60 & $1.4 \%$ & & $1.3 \%$ & \\
\hline$* \mathrm{TWC}$ & & {$[0.59,0.61]$} & {$[0.1 \%, 2.2 \%]$} & & {$[0.1 \%, 2.2 \%]$} & \\
\hline Pop $3.41 \mathrm{M}$ & Sat Mkt Share & 0.18 & $-1.2 \%$ & & $-1.2 \%$ & \\
\hline Footprint $65 \%$ & & {$[0.17,0.18]$} & {$[-1.8 \%, 0.0 \%]$} & & {$[-1.8 \%, 0.1 \%]$} & \\
\hline \multirow[t]{4}{*}{ WTP $\$ 1.65$} & Cable Carriage & 0.97 & $0.0 \%$ & & $0.0 \%$ & \\
\hline & & {$[0.84,0.97]$} & {$[-2.0 \%, 7.4 \%]$} & & {$[-2.0 \%, 7.4 \%]$} & \\
\hline & Cable Prices & 55.39 & $-0.7 \%$ & & $-0.7 \%$ & \\
\hline & & {$[54.84,56.06]$} & {$[-1.1 \%, 0.0 \%]$} & & {$[-1.1 \%, 0.0 \%]$} & \\
\hline \multirow[t]{10}{*}{ Foreclose: $0 \%$} & Aff Fees to Sat & 0.62 & $-0.2 \%$ & & $17.1 \%$ & \\
\hline & & {$[0.29,0.81]$} & {$[-5.3 \%, 6.1 \%]$} & & {$[8.8 \%, 33.3 \%]$} & \\
\hline & Cable + RSN Surplus & 18.85 & $0.0 \%$ & $0.3 \%$ & $0.1 \%$ & $1.5 \%$ \\
\hline & & {$[8.69,21.04]$} & {$[-0.1 \%, 0.5 \%]$} & {$[-1.3 \%, 5.7 \%]$} & {$[0.0 \%, 0.5 \%]$} & {$[-0.2 \%, 7.2 \%]$} \\
\hline & Satellite Surplus & 4.61 & $-1.3 \%$ & $-3.5 \%$ & $-1.7 \%$ & $-4.7 \%$ \\
\hline & & {$[1.36,4.94]$} & {$[-1.7 \%,-0.1 \%]$} & {$[-4.8 \%,-0.2 \%]$} & {$[-2.3 \%,-0.3 \%]$} & {$[-6.2 \%,-1.1 \%]$} \\
\hline & Consumer Welfare & 27.02 & $1.0 \%$ & $16.9 \%$ & $1.0 \%$ & $16.7 \%$ \\
\hline & & {$[13.49,29.81]$} & {$[0.1 \%, 1.7 \%]$} & {$[1.5 \%, 26.7 \%]$} & {$[0.0 \%, 1.7 \%]$} & {$[0.5 \%, 26.7 \%]$} \\
\hline & Total Welfare & 50.48 & $0.4 \%$ & $13.7 \%$ & $0.4 \%$ & $13.5 \%$ \\
\hline & & {$[22.93,55.42]$} & {$[0.0 \%, 0.9 \%]$} & {$[1.2 \%, 25.2 \%]$} & {$[0.0 \%, 0.9 \%]$} & {$[1.1 \%, 25.1 \%]$} \\
\hline
\end{tabular}

Notes: Simulated market outcomes across three integration scenarios. See Table 4 for details. 
Table A.11: Average Simulated Market Outcomes Across All RSNs (Satellite Adjusts Prices)

\begin{tabular}{|c|c|c|c|c|c|c|c|}
\hline & \multirow[t]{2}{*}{ (i) No VI } & \multicolumn{2}{|c|}{ (ii) VI PARs } & \multicolumn{4}{|c|}{ (iii) VI No PARs } \\
\hline & & \multicolumn{2}{|c|}{ (vs. No VI) } & \multicolumn{2}{|c|}{ (vs. No VI) } & \multicolumn{2}{|c|}{ (vs. VI PARs) } \\
\hline & Level & $\% \Delta_{l v l}$ & $\% \Delta_{W T P}$ & $\% \Delta_{l v l}$ & $\% \Delta_{W T P}$ & $\% \Delta_{l v l}$ & $\% \Delta_{W T P}$ \\
\hline \multicolumn{8}{|l|}{ ALL RSNs } \\
\hline \multirow[t]{2}{*}{ Cable Mkt Share } & 0.61 & $1.5 \%$ & & $1.5 \%$ & & $0.0 \%$ & \\
\hline & {$[0.61,0.62]$} & {$[0.2 \%, 2.1 \%]$} & & {$[0.4 \%, 2.1 \%]$} & & {$[-0.2 \%, 0.2 \%]$} & \\
\hline \multirow[t]{2}{*}{ Sat Mkt Share } & 0.19 & $0.0 \%$ & & $-0.5 \%$ & & $-0.5 \%$ & \\
\hline & {$[0.18,0.20]$} & {$[-1.3 \%, 1.1 \%]$} & & {$[-2.0 \%, 0.8 \%]$} & & {$[-1.2 \%, 0.3 \%]$} & \\
\hline \multirow[t]{2}{*}{ Cable Carriage } & 0.75 & $5.8 \%$ & & $5.4 \%$ & & $-0.4 \%$ & \\
\hline & {$[0.69,0.80]$} & {$[4.0 \%, 15.9 \%]$} & & {$[3.4 \%, 15.7 \%]$} & & {$[-2.4 \%, 1.1 \%]$} & \\
\hline \multirow[t]{2}{*}{ Cable Prices } & 55.06 & $-1.1 \%$ & & $-1.0 \%$ & & $0.1 \%$ & \\
\hline & {$[54.25,55.98]$} & {$[-1.5 \%,-0.1 \%]$} & & {$[-1.4 \%, 0.0 \%]$} & & {$[0.0 \%, 0.4 \%]$} & \\
\hline \multirow[t]{2}{*}{ Sat Prices } & 57.75 & $-0.8 \%$ & & $-1.0 \%$ & & $-0.1 \%$ & \\
\hline & {$[56.34,61.30]$} & {$[-1.5 \%,-0.1 \%]$} & & {$[-1.8 \%, 0.0 \%]$} & & {$[-0.5 \%, 0.3 \%]$} & \\
\hline \multirow[t]{2}{*}{ Aff Fees to Rivals } & 1.48 & $-1.8 \%$ & & $16.5 \%$ & & $18.2 \%$ & \\
\hline & {$[0.46,1.76]$} & {$[-5.2 \%, 1.7 \%]$} & & {$[1.9 \%, 43.0 \%]$} & & {$[8.0 \%, 50.3 \%]$} & \\
\hline \multirow[t]{2}{*}{ Cable + RSN Surplus } & 23.10 & $0.2 \%$ & $1.9 \%$ & $0.5 \%$ & $4.0 \%$ & $0.3 \%$ & $2.1 \%$ \\
\hline & {$[10.40,25.27]$} & {$[0.2 \%, 0.5 \%]$} & {$[0.9 \%, 7.5 \%]$} & {$[0.3 \%, 0.7 \%]$} & {$[1.7 \%, 14.2 \%]$} & {$[0.1 \%, 0.4 \%]$} & {$[0.4 \%, 5.5 \%]$} \\
\hline \multirow[t]{2}{*}{ Satellite Surplus } & 5.06 & $-1.9 \%$ & $-3.7 \%$ & $-2.8 \%$ & $-5.4 \%$ & $-1.0 \%$ & $-1.7 \%$ \\
\hline & {$[1.56,5.44]$} & {$[-2.8 \%,-0.5 \%]$} & {$[-9.4 \%,-0.3 \%]$} & {$[-3.6 \%,-1.2 \%]$} & {$[-12.1 \%,-0.9 \%]$} & {$[-1.3 \%,-0.5 \%]$} & {$[-3.5 \%,-0.4 \%]$} \\
\hline \multirow[t]{2}{*}{ Consumer Welfare } & 31.02 & $1.4 \%$ & $17.4 \%$ & $1.2 \%$ & $15.6 \%$ & $-0.2 \%$ & $-1.8 \%$ \\
\hline & {$[13.10,34.10]$} & {$[0.3 \%, 1.8 \%]$} & {$[2.4 \%, 33.4 \%]$} & {$[0.0 \%, 1.6 \%]$} & {$[-0.2 \%, 29.5 \%]$} & {$[-0.7 \%,-0.1 \%]$} & {$[-10.0 \%,-0.2 \%]$} \\
\hline \multirow[t]{2}{*}{ Total Welfare } & 59.17 & $0.7 \%$ & $15.7 \%$ & $0.6 \%$ & $14.2 \%$ & $-0.1 \%$ & $-1.5 \%$ \\
\hline & {$[24.85,64.45]$} & {$[0.2 \%, 0.8 \%]$} & {$[2.6 \%, 32.2 \%]$} & {$[0.1 \%, 0.8 \%]$} & {$[1.4 \%, 29.2 \%]$} & {$[-0.3 \%, 0.0 \%]$} & {$[-8.7 \%,-0.3 \%]$} \\
\hline
\end{tabular}

Notes: Average simulated market outcomes for all RSNs, weighted by the number of households in each RSN's relevant DMAs, where satellite prices are allowed to adjust. See Table 5 and Appendix C.4 for details. 


\section{B Necessary Equilibrium Conditions for Affiliate Fee Bargaining}

In this section, we describe an infinite-horizon extensive form bargaining game between channels and distributors that leads to the necessary equilibrium conditions that we employ in our analysis. This bargaining is conducted between the channel and a representative for each distributor; as noted in the main text, pricing and carriage decisions, possibly determined by other agents (e.g., local offices of each distributor), are taken as given by the bargaining agents.

Initially, assume that there are no agreements formed. In each bargaining period $t$, either the channel or the distributors make private offers (if it is the distributors, they make these offers simultaneously). An offer in bargaining period $t$ to form an agreement between the channel $c$ and distributor $f$ specifies the affiliate fee $\tau_{f c t}$. In each bargaining period, those receiving offers simultaneously announce whether they will accept or reject the offer made to them. At the end of each bargaining period, the set of agreements is observed by all players. Payoffs in a period depend on the set of agreements in force following that period's bargaining. Once an agreement is reached between $c$ and a distributor $g$, that agreement remains in force for the remainder of the game. The channel has discount factor $\delta_{c} \in(0,1)$ while each distributor has discount factor $\delta_{d} \in(0,1)$. We assume that when receiving off-equilibrium path offers the distributors have passive beliefs: that is, they continue to believe that the rival distributor has received their equilibrium offer (or no offer at all, if that is what happens on the equilibrium path).

This setup matches the structure studied by Collard-Wexler et al. (2015) and yields, when players have passive beliefs in a perfect Bayesian equilibrium, the necessary conditions that we employ in estimating our model in our cases of "No Integration" and "VI with PARs".

\section{B.1 Negative Three-Party-Surplus as a Necessary Condition for Non-Supply}

For the rest of this section, we consider a situation in which neither satellite distributor (labeled here as $g$ and $g^{\prime}$ ) is supplied with channel $c$ and we have equilibrium bundles $\mathcal{B}^{o}$, bundle prices $\boldsymbol{p}^{o}$, affiliate fees $\boldsymbol{\tau}^{o}$, and implied bundle marginal costs $\boldsymbol{m} \boldsymbol{c}^{o} .{ }^{74}$ We focus on stationary perfect Bayesian equilibria, in which continuation play depends only on the set of agreements already reached.

We now show that if three-party-surplus, given by the left-hand side of (16), is positive, then there cannot be a perfect Bayesian equilibrium with passive beliefs in the bargaining game described above in which, starting in any subgame with no deals yet reached (including at the start of the game), it is certain that no deals will be reached in the continuation game. To do so, we show that if that was the case, then at channel $c$ 's first opportunity to make an offer it could deviate and simultaneously make an affiliate fee offers $\tilde{\tau}_{g c}$ to distributor $g$ and $\tilde{\tau}_{g^{\prime} c}$ to distributor $g^{\prime}$ having the properties that:

(i) both satellite distributors anticipate greater expected profit by accepting their offer than if no agreements are reached, regardless of each satellite distributor's beliefs regarding whether the other satellite distributor will be supplied;

(ii) channel c's profits are greater if both offers are accepted than if no agreements are reached.

By hypothesis, if channel $c$ makes these offers, then - given passive beliefs - each distributor, say distributor $g$, believes that the rival distributor $g^{\prime}$ will not reach an agreement in this bargaining period. Thus, distributor $g$ believes that no deals will be reached in this period if it rejects the offer made to it, and hence no deals will occur in the continuation play either. On the other hand, if $g$ accepts, then while only $g$ will accept this period, once it has accepted channel $c$ and the rival distributor $g^{\prime}$ may reach a deal in the future. If $p_{t}$ denotes the probability that a deal is reached between channel $c$ and the rival distributor $g^{\prime}$ exactly $t$ periods after the deal with $g$ (where $p_{0} \equiv 0$ ), then $g$ 's expected payoff from acceptance is a weighted average of its payoffs when only it accepts offer $\tilde{\tau}_{g c}$ and when distributor $g$ is also immediately supplied (recall that $g$ 's payoff depends only on whether $g^{\prime}$ reaches an agreement with channel $c$, not on the level of the affilliate fee $c$ and $g^{\prime}$ agree to), where the weight on the latter payoff is $\phi_{g} \equiv \sum_{t=0} \delta_{d}^{t} p_{t}$. Thus, property (i) implies that distributor $g$ will prefer to accept channel $c$ 's offer regardless of its belief about $\phi_{g}$. Since this is true for both distributors, property (ii) implies that the deviation is profitable for channel $c$.

\footnotetext{
${ }^{74}$ For expositional convenience, we suppress the bargaining with the integrated distributor. The channel's deviation described below could be done once the channel has reached its internal agreement.
} 
In the remainder of this appendix we show that if three-party-surplus, given by the left-hand side of (16), is positive and a certain positive margin condition holds (which we verify in our empirical work), then there is a pair of affiliate fees $\left(\tilde{\tau}_{g c}, \tilde{\tau}_{g^{\prime} c}\right)$ at which properties (i) and (ii) hold. This motivates our use of negative three-party-surplus as a necessary condition for non-supply of both satellite distributors $g$ and $g^{\prime}$ to be an equilibrium, as otherwise $c$ would find it profitable to make such offers.

Notation. Define

$$
\begin{aligned}
D_{g}(\mathcal{A}) & =\sum_{m} D_{g m}\left(\mathcal{B}_{m}^{o} \cup \mathcal{A}, \boldsymbol{p}_{m}^{o}, \cdot\right), \\
\pi_{g}(\mathcal{A}) & =\sum_{m} D_{g m}\left(\mathcal{B}_{m}^{o} \cup \mathcal{A}, \boldsymbol{p}_{m}^{o}, \cdot\right) \times \underbrace{\left(p_{g m}^{o, \text { pre-tax }}-m c_{g m}^{o}\right)}_{m a r g_{g m}^{o}},
\end{aligned}
$$

to be distributor $g$ 's demand and profits when the distributor-channel pairs contained in $\mathcal{A}$ are added to all bundles; e.g., $D_{g}(g c, \emptyset)=\sum_{m} D_{g m}\left(\mathcal{B}_{m}^{o} \cup\{g c\}, \cdot\right)$ and $D_{g}\left(g c, g^{\prime} c\right)=\sum_{m} D_{g m}\left(\mathcal{B}_{m}^{o} \cup\left\{g c, g^{\prime} c\right\}, \cdot\right)$. Define

$$
\begin{aligned}
{\left[\Delta_{\mathcal{B}} D_{g}(\mathcal{A})\right] } & =\sum_{m} \underbrace{D_{g m}\left(\mathcal{B}_{m}^{o} \cup \mathcal{A}, \cdot\right)-D_{g m}\left(\mathcal{B}_{m}^{o} \cup\{\mathcal{A} \backslash \mathcal{B}\}, \cdot\right)}_{\Delta_{\mathcal{B}} D_{g m}(\mathcal{A})}, \\
{\left[\Delta_{\mathcal{B}} \pi_{g}(\mathcal{A})\right] } & =\sum_{m}\left(D_{g m}\left(\mathcal{B}_{m}^{o} \cup \mathcal{A}, \cdot\right)-D_{g m}\left(\mathcal{B}_{m}^{o} \cup\{\mathcal{A} \backslash \mathcal{B}\}, \cdot\right)\right) \times\left(p_{g m}^{o, \text { pre-tax }}-m c_{g m}^{o}\right),
\end{aligned}
$$

for $\mathcal{B} \subseteq \mathcal{A}$ to be distributor $g$ 's change in demand and profits when the distributor-channel pairs contained in $\mathcal{B}$ are removed from $\mathcal{A}$ : e.g., $\Delta_{g c} \pi_{g}\left(g c, g^{\prime} c\right)$ represents the difference in distributor $g$ 's profits from when both $g$ and $g^{\prime}$ carry channel $c$ versus when only $g^{\prime}$ carries $c$ (not including and affiliate fees paid to channel c). In terms of notation used in the main text,

$$
\begin{aligned}
\pi_{g}\left(g c, g^{\prime} c\right) & \equiv \sum_{m} \Pi_{g m}^{M}\left(\mathcal{B}_{m}^{o} \cup\left\{g c, g^{\prime} c\right\}, p_{m}^{o}, \boldsymbol{\tau}^{\prime}\right), \\
\Delta_{g c, g^{\prime} c} \pi_{g}\left(g c, g^{\prime} c\right) & \equiv \sum_{m} \Delta_{g c, g^{\prime} c} \Pi_{g m}^{M}\left(\mathcal{B}_{m}^{o} \cup\left\{g c, g^{\prime} c\right\}, p_{m}^{o}, \boldsymbol{\tau}^{\prime}\right),
\end{aligned}
$$

where $\boldsymbol{\tau}^{\prime} \equiv\left\{\boldsymbol{\tau}^{o} \cup\left(\tau_{g c}=0, \tau_{g^{\prime} c}=0\right)\right\}$

Acceptable Offers. Satellite distributor $g$ will accept an affiliate fee offer $\tilde{\tau}_{g c}$ from channel $c$ and carry the channel if its expected increase in profits from doing so exceeds the expected payments; i.e., if the following inequality holds:

$$
\left(\phi_{g} \times\left[\Delta_{g c} \pi_{g}\left(g c, g^{\prime} c\right)\right]+\left(1-\phi_{g}\right) \times\left[\Delta_{g c} \pi_{g}(g c, \emptyset)\right]\right)>\tilde{\tau}_{g c}\left(\phi_{g} \times D_{g}\left(g c, g^{\prime} c\right)+\left(1-\phi_{g}\right) \times D_{g}(g c, \emptyset)\right),
$$

where $\phi_{g} \in[0,1]$ represents distributor $g$ 's discounted probability that after accepting deviant offer $\tilde{\tau}_{g c}$ from channel $c$, the other distributor $g^{\prime}$ is also supplied. This condition is equivalent to:

$$
\tilde{\tau}_{g c}<\frac{\left(\phi_{g} \times\left[\Delta_{g c} \pi_{g}\left(g c, g^{\prime} c\right)\right]+\left(1-\phi_{g}\right) \times\left[\Delta_{g c} \pi_{g}(g c, \emptyset)\right]\right)}{\left(\phi_{g} \times D_{g}\left(g c, g^{\prime} c\right)+\left(1-\phi_{g}\right) \times D_{g}(g c, \emptyset)\right)} .
$$

Define

$$
A_{g} \equiv \frac{\left[\Delta_{g c} \pi_{g}\left(g c, g^{\prime} c\right)\right]}{D_{g}\left(g c, g^{\prime} c\right)}, \quad B_{g} \equiv \frac{\left[\Delta_{g c} \pi_{g}(g c, \emptyset)\right]}{D_{g}(g c, \emptyset)}
$$

Note that the numerators of both $A_{g}$ and $B_{g}$ are positive: i.e., the change in $g$ 's profits from carrying channel $c$ equals the increase in $g$ 's demand due to carrying channel $c$ multiplied by strictly positive margins in every market (which is the case in the data for both satellite distributors at estimated marginal costs). The derivative of the right-hand side of (17) with respect to $\phi_{g}$ is weakly positive if $A_{g} \geq B_{g}$, and strictly 
negative otherwise. Thus, if:

$$
\tilde{\tau}_{g c}(\varepsilon)=\min (\underbrace{\frac{\left[\Delta_{g c} \pi_{g}\left(g c, g^{\prime} c\right)\right]}{D_{g}\left(g c, g^{\prime} c\right)}}_{A_{g}}, \underbrace{\frac{\left[\Delta_{g c} \pi_{g}(g c, \emptyset)\right]}{D_{g}(g c, \emptyset)}}_{B_{g}})-\varepsilon,
$$

for $\varepsilon>0$, then (17) is satisfied for any $\phi_{g} \in[0,1]$, and $g$ will accept $\tilde{\tau}_{g c}(\varepsilon)$. Define $\tilde{\tau}_{g^{\prime} c}(\varepsilon)$ similarly.

Profitable for Channel $c$ to Make Offers. Consider now the decision by channel $c$ to offer both satellite distributors the set of affiliate fees $\left\{\tilde{\tau}_{g c}(\varepsilon), \tilde{\tau}_{g^{\prime} c}(\varepsilon)\right\}$ as defined in (18), where $\varepsilon>0$. Assume that the following positive margin condition holds:

$$
\left(p_{g m}^{o, \text { pre-tax }}-m c_{g m}^{o}-\tilde{\tau}_{g c}(0)\right)>0 \text { for all } m,
$$

a condition that we have verified holds for each satellite distributor in every market for every RSN when program access rules are not enforced.

We now establish that if three-party-surplus is positive, then $c$ wishes to make such offers; i.e.,.: ${ }^{75}$

$$
\begin{gathered}
\sum_{m}\left[\left[\Delta_{g c, g^{\prime} c} \Pi_{g m}^{M}\left(\left\{\mathcal{B}_{m}^{o} \cup\left\{g c, g^{\prime} c\right\}\right\}, \cdot\right)\right]+\left[\Delta_{g c, g^{\prime} c} \Pi_{g^{\prime} m}^{M}\left(\left\{\mathcal{B}_{m}^{o} \cup\left\{g c, g^{\prime} c\right\}\right\}, \cdot\right)\right] \ldots\right. \\
\left.+\left[\Delta_{g c, g^{\prime} c} \Pi_{c m}^{C}\left(\left\{\mathcal{B}_{m}^{o} \cup\left\{g c, g^{\prime} c\right\}\right\}, \cdot\right)\right]\right] \equiv E>0
\end{gathered}
$$

implies that, for sufficiently small $\varepsilon>0$,

$$
\underbrace{\sum_{m}\left[\Delta_{g c, g^{\prime} c} \Pi_{c m}^{C}\left(\left\{\mathcal{B}_{m}^{o} \cup\left\{g c, g^{\prime} c\right\}\right\}, \cdot\right)\right]+D_{g}\left(g c, g^{\prime} c\right) \tilde{\tau}_{g c}(\varepsilon)+D_{g^{\prime}}\left(g c, g^{\prime} c\right) \tilde{\tau}_{g^{\prime} c}(\varepsilon)>0}_{\tilde{\Pi}^{C}(\varepsilon)},
$$

where all profit changes are evaluated at prices $\boldsymbol{p}^{o}$ and affiliate fees $\boldsymbol{\tau}^{\prime}$. Using (19) and our previously defined notation, the left-hand side of the previous equation can be re-written as

$$
\begin{aligned}
\tilde{\Pi}^{C}(\varepsilon)=E & -\left(\left[\Delta_{g c, g^{\prime} c} \pi_{g}\left(g c, g^{\prime} c\right)\right]-D_{g}\left(g c, g^{\prime} c\right) \tilde{\tau}_{g c}(\varepsilon)\right) \\
& -\left(\left[\Delta_{g c, g^{\prime} c} \pi_{g^{\prime}}\left(g c, g^{\prime} c\right)\right]-D_{g^{\prime}}\left(g c, g^{\prime} c\right) \tilde{\tau}_{g c}(\varepsilon)\right)
\end{aligned}
$$

where the terms subtracted from $E$ on the right-hand side are the realized changes in either $g$ or $g^{\prime}$ s profits when both satellite distributors are supplied with $c$ at affiliate fees $\left\{\tilde{\tau}_{g c}(\varepsilon), \tilde{\tau}_{g^{\prime} c}(\varepsilon)\right\}$. Consider the following two cases:

- If $A_{g} \leq B_{g}$, then

$$
\begin{aligned}
& {\left[\Delta_{g c, g^{\prime} c} \pi_{g}\left(g c, g^{\prime} c\right)\right]-D_{g}\left(g c, g^{\prime} c\right) \tilde{\tau}_{g c}(\varepsilon)} \\
& =\left[\Delta_{g c} \pi_{g}\left(g c, g^{\prime} c\right)\right]+\left[\Delta_{g^{\prime} c} \pi_{g}\left(\emptyset, g^{\prime} c\right)\right]-D_{g}\left(g c, g^{\prime} c\right) \frac{\left[\Delta_{g c} \pi_{g}\left(g c, g^{\prime} c\right)\right]}{D_{g}\left(g c, g^{\prime} c\right)}+D_{g}\left(g c, g^{\prime} c\right) \varepsilon \\
& =\left[\Delta_{g^{\prime} c} \pi_{g}\left(\emptyset, g^{\prime} c\right)\right]+D_{g}\left(g c, g^{\prime} c\right) \varepsilon \\
& \leq D_{g}\left(g c, g^{\prime} c\right) \varepsilon \\
& \leq D_{g}(g c, \emptyset) \varepsilon
\end{aligned}
$$

where the third line follows because $\Delta_{g c, g^{\prime} c} \pi_{g}\left(g c, g^{\prime} c\right)=\Delta_{g c} \pi_{g}\left(g c, g^{\prime} c\right)+\Delta_{g^{\prime} c} \pi\left(\emptyset, g^{\prime} c\right)$ and from sub-

\footnotetext{
${ }^{75}$ For simplicity, we suppress the notation for the arguments of the profit functions; note, however, that, given bundle prices $\boldsymbol{p}^{o}$, the three party surplus is unaffected by the levels of $\left(\tau_{g c}, \tau_{g^{\prime} c}\right)$.
} 
stituting for $\tilde{\tau}_{g c}(\varepsilon)$ from (18), using the fact that $A_{g} \leq B_{g}$; and the final inequality follows from $g$ obtaining weakly more subscribers when $g^{\prime}$ doesn't carry $c$, which implies (under the positive margin condition) that $\left[\Delta_{g^{\prime} c} \pi_{g}\left(\emptyset, g^{\prime} c\right)\right] \leq 0$.

- If $A_{g}>B_{g}$, then:

$$
\begin{aligned}
& {\left[\Delta_{g c, g^{\prime} c} \pi_{g}\left(g c, g^{\prime} c\right)\right]-D_{g}\left(g c, g^{\prime} c\right) \tilde{\tau}_{g c}(\varepsilon)} \\
& =\left[\Delta_{g^{\prime} c} \pi_{g}\left(g c, g^{\prime} c\right)\right]+\left[\Delta_{g c} \pi_{g}(g c, \emptyset)\right]-\underbrace{\left(D_{g}\left(g c, g^{\prime} c\right)-D_{g}(g c, \emptyset)\right)}_{\left[\Delta_{g^{\prime} c} D_{g}\left(g c, g^{\prime} c\right)\right]} \tilde{\tau}_{g c}(\varepsilon)-D_{g}(g c, \emptyset) \tilde{\tau}_{g c}(\varepsilon) \\
& =\left[\Delta_{g^{\prime} c} \pi_{g}\left(g c, g^{\prime} c\right)\right]-\left[\Delta_{g^{\prime} c} D_{g}\left(g c, g^{\prime} c\right)\right] \tilde{\tau}_{g c}(\varepsilon)+\underbrace{\left[\Delta_{g c} \pi_{g}(g c, \emptyset)\right]-D_{g}(g c, \emptyset) \tilde{\tau}_{g c}(\varepsilon)}_{D_{g}(g c, \emptyset) \varepsilon} \\
& =\left[\sum_{m}\left[\Delta_{g^{\prime} c} D_{g m}\left(g c, g_{g m}^{\prime o, p r e-t a x}-m c_{g m}^{o}\right)\right]-\left[\Delta_{g^{\prime} c} D_{g}\left(g c, g^{\prime} c\right)\right] \tilde{\tau}_{g c}(\varepsilon)+D_{g}(g c, \emptyset) \varepsilon\right. \\
& =[\sum_{m} \underbrace{\left[\Delta_{g^{\prime} c} D_{g m}\left(g c, g^{\prime} c\right)\right]}_{\leq 0 \forall m} \times \underbrace{\left(p_{g m}^{o, p r e-t a x}-m c_{g m}^{o}-\tilde{\tau}_{g c}(\varepsilon)\right)}_{>0 \forall m}]+D_{g}(g c, \emptyset) \varepsilon \\
& \leq D_{g}(g c, \emptyset) \varepsilon
\end{aligned}
$$

where the fourth line follows from re-arranging terms, and the last inequality holds under the positive margin condition.

Similar conclusions apply for $g^{\prime}$ when $A_{g^{\prime}} \leq B_{g^{\prime}}$ and when $A_{g^{\prime}}>B_{g^{\prime}}$.

Substituting the inequalities in (21) and (23) for both $g$ and $g^{\prime}$ into (20) implies that:

$$
\tilde{\Pi}^{C}(\varepsilon) \geq E-\varepsilon \times\left(D_{g}(g c, \emptyset)+D_{g^{\prime}}\left(\emptyset, g^{\prime} c\right)\right) .
$$

Thus, if $\varepsilon>0, \tilde{\Pi}^{C}(\varepsilon)>0$ for any $\varepsilon \leq E /\left(D_{g}(g c, \emptyset)+D_{g^{\prime}}\left(\emptyset, g^{\prime} c\right)\right)$, and channel $c$ will find it profitable to make offers to $g$ and $g^{\prime}$ that will be accepted.

Remark. The idea behind this necessary condition is as follows. If a satellite distributor's willingness to pay for channel $c$ is lowest when the rival satellite distributor also has access to channel $c$, then the affiliate fee offers described above make each satellite distributor indifferent to accepting given that its rival will have access. But its resulting profit level is lower than when neither satellite distributor has access (granting access to just its rival lowers a satellite distributor's profit when margins are positive). As a result, if threeparty surplus from supply to both distributors is positive, channel $c$ 's profit would increase by making these offers. Suppose, instead, that a satellite distributor's' willingness to pay for channel $c$ is lowest when the rival satellite distributor does not have access to channel $c$. Then the above offers make the satellite distributor indifferent to accepting if its rival does not have access. But, when its rival does gain access (which happens with these simultaneous offers), each satellite distributor's profit falls below its level when neither has access (given positive margins) and, again, three-party surplus being positive implies that channel $c$ 's profit rises when both distributors accept.

\section{Modeling \& Estimation Details}

\section{C.1 Ownership and Control Shares}

We begin by defining the ownership variables $O_{f c t}^{M}, O_{f c t}^{C}$, and $O_{c d t}^{C C}$ that we use in our estimation, then discuss the motivation behind these choices, and finally calculate our measures in a few examples. 
Definitions. For any MVPD $f$ and channels $c$ and $d$, we define:

$$
\begin{aligned}
O_{f c t}^{M} & \equiv \frac{\sum_{j \in \mathcal{O}_{f}}\left(o_{j f t} \times o_{j c t}\right) /\left(o_{j f t}+o_{j c t}\right)}{\sum_{j \in \mathcal{O}_{f}}\left(o_{j f t}\right)^{2} /\left(o_{j f t}+o_{j c t}\right)}, \\
O_{f c t}^{C} & \equiv \frac{\sum_{j \in \mathcal{O}_{c}}\left(o_{j c t} \times o_{j f t}\right) /\left(o_{j c t}+o_{j f t}\right)}{\sum_{j \in \mathcal{O}_{c}}\left(o_{j c t}\right)^{2} /\left(o_{j c t}+o_{j f t}\right)}, \\
O_{c d t}^{C C} & \equiv \frac{\sum_{j \in \mathcal{O}_{c}}\left(o_{j c t} \times o_{j d t}\right) /\left(o_{j c t}+o_{j d t}\right)}{\sum_{j \in \mathcal{O}_{c}}\left(o_{j c t}\right)^{2} /\left(o_{j c t}+o_{j d t}\right)},
\end{aligned}
$$

where $\mathcal{O}_{g}$ represents the set of owners of firm $g$ (either MVPD or channel), o jgt represents the ownership share of firm $g$ by owner $j, O_{f c t}^{M}$ represents the ownership coefficent used by an MVPD $f$ when weighting an integrated channel $c$ 's profits, $O_{f c t}^{C}$ represents the ownership coefficent used by a channel $c$ when weighting an integrated MVPD $f$ 's profits, and $O_{c d t}^{C C}$ represents the ownership coefficent used by a channel $c$ when weighting the profits of a channel $d$ 's with which is shares a common owner.

Motivation. For the following discussion, assume that $\mu=1$.

If vertical integration always involved full ownership there would be no question of how to form firms' objective functions. The difficulty comes when there is partial ownerhsip, such as when an MVPD buys a partial stake in an RSN. In that case, the various owners of the channel may have differing preferences over the actions that the channel should take. While some papers have proposed partial ownership measures (e.g., Bresnahan and Salop, 1986; O'Brien and Salop, 2000), little is known empirically about how conflicting objectives among a firm's owners translates into the firm's behavior.

Our approach to this issue and resulting measures can be understood as follows: consider, as an example, a channel $c$ that is partially owned by an MVPD $f$ (and owned by no other entity with ownership stakes in another distributor or channel). Denote the channel-specific profits as $\pi_{c}$ and MVPD-specific distribution profits as $\pi_{f}$. We assume that (the manager of) channel $c$ maximizes an objective that is an ownership-share weighted average (with weights representing "shares of control") of its owners' "normalized" preferences over channel and MVPD distribution profits:

$$
\begin{aligned}
\tilde{\Pi}_{c t} & =\sum_{j} \underbrace{o_{j c t}}_{\text {Ownership Shares }}[\underbrace{\frac{o_{j c t}}{o_{j c t}+o_{j f t}} \pi_{c}+\frac{o_{j f t}}{o_{j c t}+o_{j f t}} \pi_{f}}_{\text {Relative "Cash Flows" }}] \\
& \propto \pi_{c}+O_{f c t}^{C} \times \pi_{f},
\end{aligned}
$$

where the normalization for each owner $j$ places weights on $\pi_{c}$ and $\pi_{f}$ that sum to 1 . Similar logic underlies the other ownership variables $\left(O_{f c t}^{M}\right.$ and $\left.O_{c d t}^{C C}\right)$.

One can imagine various approaches to this issue. Our measures differ from those used in the literature cited above on partial ownership by normalizing cash-flows for each owner (e.g., in (24), the weights on $\pi_{c}$ and $\pi_{f}$ for each owner $j$ sum to 1), and in using ownership shares as the control weights. Recent work by Azar et al. (2016), for example, uses ownership shares as control weights as we do, but does not normalize the cash flows. Absent this normalization, in the above example channel $c$ would maximize instead an objective proportional to $\pi_{c}+\tilde{O}_{f c t}^{C} \pi_{f}$, where $\tilde{O}_{f c t}^{C}=\left(\sum_{j \in \mathcal{O}_{c}} o_{j c t} \times o_{j f t}\right) /\left(\sum_{j \in \mathcal{O}_{c}} o_{j c t}^{2}\right)$. Of course, these two approaches do not exhaust the possibilities. For example, one could assume that a firm's owners bargain efficiently, resulting in behavior that maximizes their joint payoff. In that case, channel $c$ would maximize an objective proportional to $\pi_{c}+\bar{O}_{f c t}^{C} \pi_{f}$, where $\bar{O}_{f c t}^{C}=\left(\sum_{j \in \mathcal{O}_{c}} o_{j f t}\right) /\left(\sum_{j \in \mathcal{O}_{c}} o_{j c t}\right)$.

One reason that we depart from these two approaches is that these other measures can lead to some counterintuitive predictions. For example, consider a situation in which an MVPD owns share $x$ of channel $c$, while $N$ other shareholders each own share $(1-x) / N$ of channel $c$ and nothing else. In that case, $\tilde{O}_{f c t}^{C}=x /\left(x^{2}+(1-x)^{2} / N\right), \bar{O}_{f c t}^{C}=1$, and our measure is $O_{f c t}^{C}=x$. As $N$ goes to $\infty$, the first measure $\tilde{O}_{f c t}^{C} \rightarrow 1 / x$. That is, no matter how small the MVPD's ownership share $x$ is, as $N$ gets large the channel's behavior comes to be what the MVPD would want. The $N$ shareholders with common interests are essentially powerless. Indeed, for small $x$ the channel simply maximizes the MVPD's distribution profits. This outcome 
puts even more weight on the MVPD's distribution profits than the jointly efficient weight $\bar{O}_{f c t}^{C}$, which leads the channel to maximize $\left(\pi_{c}+\pi_{f}\right)$ regardless of how small the MVPD's ownership share $x$ is. In contrast, in this example our measure puts a weight on the MVPD's distribution profits that equals the MVPD's ownership share in the channel (which is less than the jointly efficient weight).

We next prove an important feature of our measure: if an MVPD and channel share at most a single common owner (i.e., an entity that has positive ownership stakes in both firms), then $O_{f c t}^{M}=O_{f c t}^{C}$.

Lemma 1 Consider MVPD $f$ and channel $c$. If there exists at most one owner $j$ such that $o_{j f t} \times o_{j c t}>0$, then $O_{f c t}^{M}=O_{f c t}^{C}$.

Proof. Let $o_{j c t}=x$ and $o_{j f t}=y$. The numerators for $O_{f c t}^{M}$ and $O_{f c t}^{C}$ are equivalent. The denominator for $O_{f c t}^{M}$ equals $y^{2} /(x+y)+(1-y)$. The denominator for $O_{f c t}^{C}$ equals $x^{2} /(x+y)+(1-x)$. Both equal $(x+y-x y) /(x+y)$.

This property holds for all MVPD and channel pairs that we consider in our analysis.

Finally, note one important empirical advantage of our measure $O_{f c t}^{C}$ : it is invariant to the distribution of ownership among owners with no ownership interests in any other firms within the industry. For example, in the above example, we would also have $O_{f c t}^{C}=x$ if instead there was a single firm owning the $(1-x)$ share of channel $c$ (and nothing else). As a result, we do not need data on the pattern of ownership except that among firms who are vertically integrated.

Examples. We provide two examples of our ownership variables.

1. Unitary MVPD ownership. Consider an MVPD $f$ that owns $o_{f c t}=x$ share of channel $c$. In this case, there is a single owner $j$ of MVPD $f$, where $o_{j f t}=1$. Then the MVPD $f$ places weight $O_{f c t}^{M}$ on channel $c$ 's profits (relative to its own profits) when making decisions, where:

$$
O_{f c t}^{M}=\frac{(1 \times x) /(1+x)}{(1) /(1+x)}=x .
$$

For the channel $c$, there are essentially two owners: one that owns $(1-x)$ of $c$ (and none of MVPD $f$ ), and MVPD $f$ that owns $x$ of $c$. Here, channel $c$ places weight $O_{f c t}^{C}$ on MVPD $f$ 's profits (relative to its own profits) when making decisions, where:

$$
O_{f c t}^{C}=\frac{(x \times 1) /(1+x)}{(1-x)^{2} /(1-x)+(x)^{2} /(1+x)}=\frac{x}{(1+x)(1-x)+x^{2}}=x .
$$

Thus, $O_{f c t}^{M}=O_{f c t}^{C}$ when a channel only has a single integrated owner.

2. Channel Conglomerates. Assume that a 3rd party owns $x$ share of channel $c$ and $y$ share of channel $d$. Then channel $c$ places weight $O_{c d t}^{C C}$ on channel $d$ 's profits (relative to its own profits) when making decisions, where:

$$
O_{c d t}^{C C}=\frac{(x \times y) /(x+y)}{(1-x)^{2} /(1-x)+(x)^{2} /(x+y)}=\frac{x \times y}{(x+y)(1-x)+x^{2}}=\frac{x \times y}{x+y-x \times y} .
$$

\section{C.2 Solving for Negotiated Affiliate Fees and Bundle Marginal Costs}

We omit the subscript on $\Psi_{f c t} \equiv\left(1-\zeta_{f c t}\right) / \zeta_{f c t}$ for the expressions in this subsection. Let $\mathcal{B}_{f m t}^{R}$ be the observed set of RSNs carried by $f$ in market $m$ in period $t$.

Consider MVPD $f$ bargaining with channel $c$ over affiliate fee $\tau_{f c t}$, where $c$ has at most a single integrated 
owner. Closed form expressions for MVPD and channel "GFT" terms in (9) are:

$$
\begin{aligned}
G F T_{f c t}^{M}= & \sum_{m \in \mathcal{M}_{f c t}}\left[\left[\mu_{f c t} D_{f m t}-D_{f m t}^{\backslash f c}\right] \tau_{f c t}+\mu_{f c t} \times\left(D_{f m t}+\sum_{g \neq f: c \in \mathcal{B}_{g m t}}\left[\Delta_{f c} D_{g m t}\right]\right) a_{c m t}+\mu_{f c t} \sum_{g \neq f: c \in \mathcal{B}_{g m t}}\left[\Delta_{f c} D_{g m t}\right] \tau_{g c t}\right. \\
& \left.+\sum_{d \in \mathcal{V}_{f t} \backslash c} \sum_{g \in \mathcal{F}_{m t}: d \in \mathcal{B}_{g m t}}\left[\Delta_{f c} D_{g m t}\right] \mu_{f d t} \times\left(\tau_{g d t}+a_{d m t}\right)+\left[\Delta_{f c} D_{f m t}\right]\left(p_{f m t}^{\text {pre-tax }}-m c_{f m t}\right)\right], \\
G F T_{f c t}^{C}= & \sum_{m \in \mathcal{M}_{f c t}}\left[\left(D_{f m t}-\mu_{f c t} D_{f m t}^{\backslash f c}\right) \tau_{f c t}+\left(D_{f m t}+\sum_{g \neq f: c \in \mathcal{B}_{g m t}}\left[\Delta_{f c} D_{g m t}\right]\right) a_{c m t}+\sum_{g \neq f: c \in \mathcal{B}_{g m t}}\left[\Delta_{f c} D_{g m t}\right]\left(\tau_{g c t}\right)\right. \\
& \left.+\sum_{g \in \mathcal{F}_{m t}} \lambda_{R: f c t}\left[\Delta_{f c} D_{g m t}\right] \sum_{d \in \mathcal{B}_{g m t} \backslash c} \mu_{c d t}^{C C} \times\left(\tau_{g d t}+a_{d m t}\right)+\sum_{g \in \mathcal{F}_{m t}} \mu_{g c t} \lambda_{R: f c t}\left[\Delta_{f c} D_{g m t}\right]\left(p_{g m t}^{\text {pre-tax }}-m c_{g m t}\right)\right],
\end{aligned}
$$

where: $D_{f m t}^{\backslash f c}$ is the demand for $f$ in market $m$ if it dropped channel $c$; $\lambda_{R: f c t}=\lambda_{R}$ if $f$ and $c$ are not integrated, and $\lambda_{R: f c t}=1$ otherwise; $\mu_{f c t}=\mu \times O_{f c t}^{M} ; \mu_{c d t}^{C C}=\mu \times O_{c d t}^{C C}$; and $\mathcal{V}_{f t} \equiv\left\{c: O_{f c t}>0\right\}$ is the set of channels owned by MVPD $f$ in period $t$.

Focus on the bargain between an RSN $c$ and MVPD $f .{ }^{76}$ Using (25) and (26), the Nash Bargaining first-order condition $\forall f \in \mathcal{F}_{m t}, c \in \mathcal{C}_{t}^{R}$ given by (10) $\left(G F T_{f c t}^{C}=\Psi G F T_{f c t}^{M}\right)$ can be re-written as:

$$
\begin{aligned}
\tau_{f c t} & \sum_{m \in \mathcal{M}_{f c t}}\left[(1+\Psi)\left(1-\mu_{f c t}\right) D_{f m t}\right]+\sum_{g \neq f: c \in \mathcal{B}_{g m t}} \tau_{g c t} \sum_{m \in \mathcal{M}_{f c t}}\left(1-\Psi \mu_{f c t}\right)\left[\Delta_{f c} D_{g m t}\right] \\
+ & \sum_{g \in \mathcal{F}_{m t}} \sum_{d \in \mathcal{B}_{g m t} \backslash c} \tau_{g d t} \times\left(\left(\Psi-\mu_{f c t}\right) \mathbb{1}_{g=f}+\mu_{c d t}^{C C}-\Psi \mu_{f d t}\right) \sum_{m \in \mathcal{M}_{f c t}}\left[\Delta_{f c} D_{g m t}\right]+\left(\Psi-\mu_{f c t}\right) \sum_{m \in \mathcal{M}_{f c t}} m c_{f m t}^{\backslash R}\left[\Delta_{f c} D_{f m t}\right]= \\
& \sum_{m \in \mathcal{M}_{f c t}}\left[\left(\Psi-\mu_{f c t}\right)\left[\Delta_{f c} D_{f m t}\right] p_{f m t}^{\mathrm{pre}-\operatorname{tax}}\right]-\sum_{m \in \mathcal{M}_{f c t}}\left[a_{c m t} \times\left(\left(1-\Psi \mu_{f c t}\right) D_{f m t}+\left(1-\Psi \mu_{f c t}\right) \sum_{g \neq f: c \in \mathcal{B}_{g m t}}\left[\Delta_{f c} D_{g m t}\right]\right)\right. \\
& \left.+\sum_{g \in \mathcal{F}_{m t}} \sum_{d \in \mathcal{B}_{g m t} \backslash c} a_{d m t} \times\left(\mu_{c d t}^{C C}-\Psi \mu_{f d t}\right)\left(\left[\Delta_{f c} D_{g m t}\right]\right)\right]
\end{aligned}
$$

where $m c_{f m t}^{\backslash R}$ represents non-RSN marginal costs: i.e., $m c_{f m t}^{\backslash R} \equiv m c_{f m t}-\sum_{d \in \mathcal{B}_{f m t}^{R}} \tau_{f d t}$.

We can also re-write the pricing first-order condition in (5), which provides the optimal set of prices for every cable provider $f$ in every market $m$, as:

$$
\begin{aligned}
\sum_{g \in \mathcal{F}_{m t}} \frac{\partial D_{g m t}}{\partial p_{f m t}}\left(m c_{g m t}^{\backslash R} \mathbb{1}_{g=f}+\sum_{d \in \mathcal{B}_{g m t}^{R}}\left(\mathbb{1}_{g=f}-\mu_{f d t}\right) \tau_{g d t}\right)= \\
{\left[\frac{D_{f m t}}{1+\operatorname{tax}_{f m t}}+\frac{\partial D_{f m t}}{\partial p_{f m t}} p_{f m t}^{\text {pre-tax }}+\sum_{g \in \mathcal{F}_{m t}} \frac{\partial D_{g m t}}{\partial p_{f m t}} \sum_{d \in \mathcal{B}_{g m t}^{R}} \mu_{f d t} a_{d m t}\right] . }
\end{aligned}
$$

However, if $f$ is a satellite provider (denoted $f \in \mathcal{F}^{\text {sat }}$ ), we assume that there is a single national price $p_{f t}$ and non-RSN marginal cost $\hat{m} c_{f m t}^{\backslash R}$ that applies across all markets; this implies that there is only a single pricing first-order condition for satellite firms:

$$
\begin{aligned}
\sum_{m} \sum_{g \in \mathcal{F}_{m t}} \frac{\partial D_{g m t}}{\partial p_{f t}}\left(m c_{g t}^{\backslash R} \mathbb{1}_{g=f}+\sum_{d \in \mathcal{B}_{g m t}^{R}}\left(\mathbb{1}_{g=f}-\mu_{f d t}\right) \tau_{g d t}\right)= \\
\sum_{m}\left(\frac{D_{f m t}}{1+\operatorname{tax}_{f m t}}+\frac{\partial D_{f m t}}{\partial p_{f t}} p_{f t}^{\text {pre-tax }}+\sum_{g \in \mathcal{F}_{m t}} \frac{\partial D_{g m t}}{\partial p_{f m t}} \sum_{d \in \mathcal{B}_{g m t}^{R}} \mu_{f d t} a_{d m t}\right) \quad \forall f \in \mathcal{F}^{s a t} .
\end{aligned}
$$

\footnotetext{
${ }^{76}$ In estimation, we are assuming that $\lambda_{R}=0$ in the "non-loophole" markets, and thus omit terms that would otherwise enter (e.g., if $c$ were integrated with a rival MVPD $f^{\prime}$ ). In the counterfactuals, we re-introduce these terms.
} 
Equations (27), (28), and (29) express affiliate fees and marginal costs as a function of demand parameters, prices, and advertising rates. We thus solve for the vector of RSN affiliate fees $\left\{\tau_{f c t}\right\}_{\forall f, t, c \in \mathcal{C}_{t}^{R}}$ for all RSNs and non-RSN bundle marginal costs $\left\{m c_{f m t}^{\backslash R}\right\}_{\forall f m t}$ via matrix inversion when evaluating the objective for any parameter vector $\boldsymbol{\theta}$.

National Channels. We use our estimates of RSN affiliate fees and non-RSN bundle marginal costs to recover $\left\{\tau_{f c t}\right\}_{\forall f t, c \notin \mathcal{C}_{t}^{R}}$ for non-RSN channels via matrix inversion on the following:

$$
\begin{aligned}
\tau_{f c t} & \sum_{m \in \mathcal{M}_{f c t}}\left[D_{f m t}+\Psi D_{f m t}^{\backslash f c}\right]+\sum_{g \neq f: c \in \mathcal{B}_{g m t}} \tau_{g c t} \sum_{m \in \mathcal{M}_{f c t}}\left[\Delta_{f c} D_{g m t}\right]= \\
& \sum_{m \in \mathcal{M}_{f c t}}\left[\Psi \times\left[\Delta_{f c} D_{f m t}\right]\left(p_{f m t}^{\mathrm{pre}-\mathrm{tax}}-\hat{m} c_{f m t}\right)\right]+\sum_{g \in \mathcal{F}_{m t}} \sum_{d \in \mathcal{B}_{g m t} \backslash c} \mu_{f d t} \Psi \hat{\tau}_{g d t} \sum_{m \in \mathcal{M}_{f c t}}\left[\Delta_{f c} D_{g m t}\right] \\
& -\sum_{m \in \mathcal{M}_{f c t}}\left[a_{c m t} \times\left(D_{f m t}+\sum_{g \neq f: c \in \mathcal{B}_{g m t}}\left[\Delta_{f c} D_{g m t}\right]\right)+\sum_{g \in \mathcal{F}_{m t}} \sum_{d \in \mathcal{B}_{g m t} \backslash c} a_{d m t} \times\left(-\Psi \mu_{f d t}\right)\left(\left[\Delta_{f c} D_{g m t}\right]\right)\right],
\end{aligned}
$$

where we construct estimates of each bundle's marginal costs from our recovered non-RSN marginal costs as follows: $\hat{m} c_{f m t} \equiv \hat{m} c_{f m t}^{\backslash R}+\sum_{d \in \mathcal{B}_{f m t}^{R}} \hat{\tau}_{f d t}$. We assume away integration incentives for non-RSNs so that $\mu_{f c t}=0 \forall f t, c \notin \mathcal{C}_{t}^{R}$.

\section{C.3 Further Estimation and Simulation Details}

Construction of Disagreement Payoffs. Computation of several moments in estimation require constructing values of $\Delta_{f c}\left[\Pi_{f m t}^{M}(\cdot)\right]$ and $\Delta_{f c}\left[\Pi_{c m t}^{C}(\cdot)\right]$ for each MVPD $f$ and channel $c$ that contract in each period. These "gains from trade" for each pair are functions of both agreement and disagreement profits. Profits from agreement (as a function of $\boldsymbol{\theta}$ ) are computed using (4) and (7) with observed prices and bundles. Consistent with our timing assumptions (i.e., bundle prices, channel carriage, and affiliate fees are simultaneously determined), profits from disagreement between MVPD $f$ and channel $c$ are computed by removing $c$ from all bundles offered by $f$ and holding fixed: (i) bundle prices for all cable and satellite MVPDs; (ii) carriage decisions for other MVPDs $\left(\mathcal{B}_{g m t}^{\prime}=\mathcal{B}_{g m t} \forall g \neq f\right)$; and (iii) affiliate fees $\hat{\tau}_{-f c, t}$ for all other MVPD-channel pairs.

Importance Sampling. We follow the importance sampling approach of Ackerberg (2009) to estimate our model. We begin by simulating 350 households per market from an initial distribution of random preferences, so that each household is characterized by a vector of preferences for each channel and satellite distributor. For each of these simulated households, we solve the viewership problem given by (1) for each downstream firm in the household's market. To evaluate candidate parameter vectors in the estimation objective function, we approximate the relevant integrals (e.g., for implied market shares or mean viewership by channel) by weighting the initial simulated households by the implied importance sampling weights that depend on the initial distribution and the candidate distribution. For example, if one were to draw from an $N(0,1)$ distribution initially, and want to approximate the mean of an $N(0.5,1)$ distribution, one would put relatively more weight on the initial draws near 0.5 , and relatively less weight on negative draws.

The approximation is more accurate the closer is the initial distribution to the candidate distribution. Therefore, we iteratively updated the initial distribution several times through the process. That is, after moving in the parameter space to lower objective function values, we re-simulated an initial distribution from the distribution of preferences implied by the then current best parameter vector.

Estimation of Channel Decay Parameters. We allow for households to have variance in their values of $\nu^{S}$ in order to estimate this parameter using importance sampling. Without allowing for variance in $\nu^{S}$, we would not be able to obtain any benefits of the importance sampling procedure as we would have to resolve the viewership problem for each simulated household at each objective function valuation. We 
assume that households' had a value of $\nu^{S}$ drawn from a normal distribution with a common mean and standard deviation of 0.015 .

As discussed in footnote 48 , we estimate $\nu^{N S}$ on a coarse grid (with values contained in $\{0.8,0.91,0.95,0.99\}$ ); the objective function was minimized for $\nu^{N S}=0.95$. The computational difficulty of estimating $\nu^{N S}$ using the same procedure as with $\nu^{S}$ is the following: with positive variance in both $\nu^{S}$ and $\nu^{N S}$, and given that values of channel viewership utilities $\rho$ are independent of decay parameters, there would commonly be households whose parameter draws implied very unrealistic viewership patterns (e.g., spending $90 \%$ of their full day watching a single channel). Such outlier households would imply very inelastic demand for cable or satellite bundles, and consequently implausibly high mark-ups in certain markets. Although these households would have negligible weight absent simulation error, memory and computational limitations prevented us from using more than 350 household simulations per market in estimation.

To examine the sensitivity of our results to different values of $\nu^{N S}$, we have also computed our counterfactual simulations using parameter estimates obtained when $\nu^{N S}=0.91$ and $\nu^{N S}=0.99$. In both cases, we find that average simulated changes in surplus or welfare across all RSNs are not statistically different from those reported in Table 5 (which are computed using parameter estimates obtained when $\nu^{N S}=0.95$ ); and our main findings do not change.

\section{C.4 Computing Counterfactual Equilibria}

For each RSN channel $c$ and each integration scenario - no vertical integration, vertical integration with PARs, and vertical integration without PARs - we compute predicted outcomes in all of the RSN's relevant markets in 2007. We also recompute outcomes for the integration scenario that is "observed" in the data for each RSN.

We maintain the following assumptions: (i) if supplied, satellite distributors carry $c$ in all of $c$ 's relevant markets; (ii) cable carriage decisions and affiliate fees for $c$ are allowed to adjust, but those for all other channels are held fixed. In our main counterfactual results (reported in the main text and in Tables A.5A.10), we allow only cable prices to adjust in each RSN's relevant markets and hold fixed national satellite prices at levels observed in the data. In a robustness test, we allow satellite prices to adjust under the assumption that they are set at the DMA level; results from this specification are reported in Table A.11.

As discussed in the main text and in footnote 61 , if we are examining the vertical integration scenario without PARs, we also allow for the channel's supply decision to adjust: e.g., if RSN $c$ is a cable-owned RSN (or is non-integrated and assigned a cable owner under the vertical integration scenarios), we compute outcomes under four "supply scenarios" - the channel is supplied to both satellite distributors, supplied to only DirecTV or Dish, or supplied to neither satellite distributor - and test which supply scenario is robust to deviations by the channel.

For each RSN $c$, integration scenario, supply scenario, and set of carriage disturbances $\left\{\Delta_{f c} \omega_{f m t}\right\}_{f, m},{ }^{77}$ we compute outcomes in all of the RSN's relevant markets by iterating over the following procedure until prices, fees, and carriage decisions converge: ${ }^{78}$

1. Given affiliate fees and carriage decisions, we update bundle prices for all cable (and satellite, when appropriate) distributors to maximize profits.

2. Given bundle prices and carriage decisions, we update affiliate fees $\left\{\tau_{f c t}^{C F}\right\}_{f}$ using the following system

\footnotetext{
${ }^{77}$ We draw a vector of carriage disturbances $\left\{\Delta_{f c} \omega_{f m t}\right\}_{f, m}$ for all MVPDs and relevant markets for RSN $c$, where each element $\Delta_{f c} \omega_{f m t}$ is drawn from a truncated normal distribution with variance $4 \hat{\sigma}_{\omega}^{2}$ to rationalize observed carriage decisions in the data given by (14). I.e., for every market $m$ where $c \in \mathcal{B}_{f m t}$, we draw $\Delta_{f c} \omega_{f m t}$ conditional on it being less than $\Delta_{f c} \pi_{f m t}^{M}\left(\mathcal{B}_{m t}, \cdot\right)$; and for every market $m$ where $c \notin \mathcal{B}_{f m t}$, we draw $\Delta_{f c} \omega_{f m t}$ conditional on it being greater than $\Delta_{f c} \pi_{f m t}^{M}\left(\mathcal{B}_{m t} \cup f c, \cdot\right)$. All counterfactuals outcomes are computed for and averaged over 10 sets of carriage disturbance draws.

${ }^{78}$ We iterate until the sum of absolute differences between all RSN affiliate fees and all downstream prices does not change by more than $10^{-} 3$.
} 
of equations:

$$
\begin{aligned}
& \tau_{f c t}^{C F} \sum_{m \in \mathcal{M}_{f c t}}\left[(1+\Psi)\left(1-\mu_{f c t}\right) D_{f m t}^{C F}\right]+\sum_{g \neq f: c \in \mathcal{B}_{g m t}^{R, C F}} \tau_{g c t}^{C F} \sum_{m \in \mathcal{M}_{f c t}}\left(1-\Psi \mu_{f c t}-\mu_{g c t} \lambda_{R}\right)\left[\Delta_{f c} D_{g m t}^{C F}\right] \\
& +\sum_{g \in \mathcal{F}_{m t}} \sum_{d \in \mathcal{B}_{g m t}^{R, C F} \backslash c} \tau_{g d t}^{C F} \times\left(\left(\Psi-\mu_{f c t}\right) \mathbb{1}_{g=f}+\mu_{c d t}^{C C}-\Psi \mu_{f d t}-\mu_{g c t} \lambda_{R}\right) \sum_{m \in \mathcal{M}_{f c t}}\left[\Delta_{f c} D_{g m t}^{C F}\right]= \\
& \sum_{m \in \mathcal{M}_{f c t}}\left[\left(\Psi-\mu_{f c t}\right)\left(p_{f m t}^{\mathrm{pre}-\mathrm{tax}, C F}-\hat{m} c_{f m t}^{\backslash R}\right)\left[\Delta_{f c} D_{f m t}^{C F}\right]-\mu_{f^{\prime} c t} \lambda_{R} \times\left(p_{f^{\prime} m t}^{\mathrm{pre-tax}, C F}-\hat{m c} c_{f^{\prime} m t}^{\backslash R}\right)\left[\Delta_{f c} D_{f^{\prime} m t}^{C F}\right]\right] \\
& -\sum_{m \in \mathcal{M}_{f c t}}\left[a_{c m t} \times\left(\left(1-\Psi \mu_{f c t}\right) D_{f m t}^{C F}+\left(1-\Psi \mu_{f c t}\right) \sum_{g \neq f: c \in \mathcal{B}_{g m t}^{R, C F}}\left[\Delta_{f c} D_{g m t}^{C F}\right]\right)\right. \\
& \left.+\sum_{g \in \mathcal{F}_{m t}} \sum_{d \in \mathcal{B}_{g m t}^{R, C F} \backslash c} a_{d m t} \times\left(\mu_{c d t}^{C C}-\Psi \mu_{f d t}\right)\left(\left[\Delta_{f c} D_{g m t}^{C F}\right]\right)\right] \quad \forall f, c,
\end{aligned}
$$

where $f$ and $f^{\prime}$ represent the MVPDs with which $c$ is potentially integrated. Equation (31) differs from (27) insofar that we now allow for the possibility that $\lambda_{R}>0$, and that $c$ may be integrated with a rival MVPD $f^{\prime}$ when bargaining with $f$. We only update $\left\{\tau_{f c t}\right\}_{\forall f}$ for the given channel $c$ that is being examined, and not for other channels $d$ that may be active in $c$ 's relevant markets.

3. Given bundle prices, affiliate fees, and carriage disturbances, we update carriage decisions by checking in each relevant market whether or not the cable distributor wishes to carry the channel using (14). 